\title{
Synthesis, Resolution, and Aldol Reactions of a Planar-Chiral Lewis Acid Complex
}

\author{
Shih-Yuan Liu, Ivory D. Hills, and Gregory C. Fu* \\ Department of Chemistry, Massachusetts Institute of Technology \\ Cambridge, MA 02139
}

Supporting Information

\section{General}

All oxygen- and moisture-sensitive manipulations were carried out under an inert atmosphere using either standard Schlenk techniques or a glove box.

All chemicals and solvents were purchased from Aldrich or Strem Chemicals and used as received. Iron complex $( \pm)-2^{1}$ was prepared according to a literature procedure.

THF, $\mathrm{Et}_{2} \mathrm{O}, \mathrm{CH}_{2} \mathrm{Cl}_{2}$, and toluene were purified by passing through a neutral alumina column under argon. Anhydrous pentane and benzene (Aldrich) were used as received.

Silica gel (230-400 mesh; SiliCycle) was heated under vacuum in a $200{ }^{\circ} \mathrm{C}$ sand bath for $12 \mathrm{~h}$. Flash chromatography was performed with this pre-treated silica gel under an inert atmosphere.

11B NMR spectra were recorded on a Varian Unity 300 or on a Varian Unity 500 spectrometer at ambient temperatur and are referenced to boron trifluoride diethyl etherate $(\delta 0)$. 


\section{Synthesis and Resolution of Planar-Chiral Lewis Acid 1 (Figure 2)}

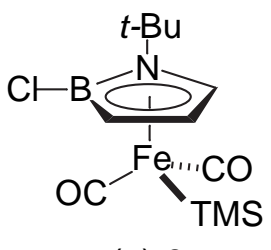

$( \pm)-3$

In a glove box, eight vials were each charged with $( \pm)-2(100 \mathrm{mg}, 0.253 \mathrm{mmol})$ and sodium amalgam $(10 \% \mathrm{Na}$ content; $570 \mathrm{mg}, 2.53 \mathrm{mmol} \mathrm{Na})$. THF $(1.0 \mathrm{~mL})$ was added to each vial, and the resulting heterogeneous mixtures were stirred vigorously for $25 \mathrm{~min}$. Then, the dark reaction mixtures were combined and filtered through an acrodisc. The residue was extracted with additional THF $(24 \mathrm{~mL})$ and then filtered. TMSCl (550 mg, $5.06 \mathrm{mmol}$ ) was added dropwise to the combined THF solutions, with stirring. After 30 min, the reaction mixture was concentrated, and then the crude mixture was subjected to flash chromatography (pentane). The desired product eluted as the first yellow band. Yellow solid (440 mg, 64\%).

This reaction was run in eight vials, rather than one, because the results were not always reproducible on a larger scale.

${ }^{1} \mathrm{H}$ NMR $\left(500 \mathrm{MHz}, \mathrm{CD}_{2} \mathrm{Cl}_{2}\right): \delta 5.72(\mathrm{~s}, 1 \mathrm{H}), 4.79\left(\mathrm{~d},{ }^{3} J_{\mathrm{HH}}=3.5 \mathrm{~Hz}, 1 \mathrm{H}\right), 3.40\left(\mathrm{~d},{ }^{3} J_{\mathrm{HH}}=\right.$ $4.5 \mathrm{~Hz}, 1 \mathrm{H}), 1.45(\mathrm{~s}, 9 \mathrm{H}), 0.26(\mathrm{~s}, 9 \mathrm{H})$.

${ }^{13} \mathrm{C}$ NMR $\left(125 \mathrm{MHz}, \mathrm{CD}_{2} \mathrm{Cl}_{2}\right): \delta$ 215.2, 214.0, 90.4, 86.0, 71.4 (br), 57.7, 31.0, 7.7.

${ }^{11} \mathrm{~B}$ NMR $\left(160 \mathrm{MHz}, \mathrm{CD}_{2} \mathrm{Cl}_{2}\right): \delta 19.9$.

FTIR (thin film) 3157, 2947, 2889, 1980, 1921, 1470, 1434, 1401, 1371, 1325, 1268, 1241, $1203,1174,1130 \mathrm{~cm}^{-1}$.

HRMS (EI) calcd for $\mathrm{C}_{12} \mathrm{H}_{21} \mathrm{BClFeNO} \mathrm{Si}_{2}\left(\mathrm{M}^{+}\right) 341.0473$, found 341.0467 .

$\operatorname{mp} 65-66{ }^{\circ} \mathrm{C}$.

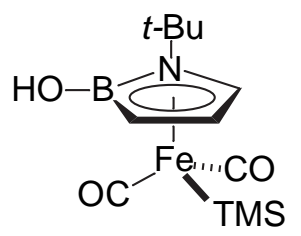

$( \pm)-4$

In a glove box, a vial was charged with $( \pm)-3(480 \mathrm{mg}, 1.41 \mathrm{mmol})$ and $\mathrm{K}_{3} \mathrm{PO}_{4}$ monohydrate $(971 \mathrm{mg}, 4.22 \mathrm{mmol})$. THF $(19.0 \mathrm{~mL})$ was added, and the reaction mixture was stirred vigorously for $9 \mathrm{~h}$. Then, pentane was added, and the reaction mixture was filtered through an acrodisc. The filtrate was concentrated under vacuum, and the resulting residue was purified by flash chromatography (pentane $/ \mathrm{Et}_{2} \mathrm{O}$ ), which furnished the desired product as a yellow oil $(294 \mathrm{mg}, 65 \%)$.

${ }^{1} \mathrm{H} \mathrm{NMR}\left(500 \mathrm{MHz}, \mathrm{CD}_{2} \mathrm{Cl}_{2}\right): \delta 5.54(\mathrm{~s}, 1 \mathrm{H}), 4.62\left(\mathrm{~d},{ }^{3} \mathrm{~J}_{\mathrm{HH}}=4.5 \mathrm{~Hz}, 1 \mathrm{H}\right), 3.44(\mathrm{~s}, 1 \mathrm{H})$, $2.78\left(\mathrm{~d},{ }^{3} \mathrm{~J}_{\mathrm{HH}}=5.0 \mathrm{~Hz}, 1 \mathrm{H}\right), 1.36(\mathrm{~s}, 9 \mathrm{H}), 0.21(\mathrm{~s}, 9 \mathrm{H})$.

${ }^{13} \mathrm{C}$ NMR $\left(125 \mathrm{MHz}, \mathrm{CD}_{2} \mathrm{Cl}_{2}\right): \delta$ 216.6, 215.0, 88.2, 82.8, 58.5 (br), 55.6, 30.5, 7.4.

${ }^{11} \mathrm{~B}$ NMR $\left(160 \mathrm{MHz}, \mathrm{CD}_{2} \mathrm{Cl}_{2}\right): \delta 21.5$. 
FTIR (thin film) 3625, 2947, 2889, 1968, 1895, 1470, 1434, 1410, 1367, 1280, 1239, 1208, $1169 \mathrm{~cm}^{-1}$.

HRMS (EI) calcd for $\mathrm{C}_{12} \mathrm{H}_{22} \mathrm{BFeNO}_{3} \mathrm{Si}\left(\mathrm{M}^{+}\right)$323.0806, found 323.0811.

This compound can be assayed via chiral HPLC (Daicel Chiralpak OD, hexanes:i$\mathrm{PrOH}=95: 5,1.0 \mathrm{~mL} / \mathrm{min},(\mathrm{R})$ isomer: $5.6 \mathrm{~min},(\mathrm{~S})$ isomer: $7.3 \mathrm{~min})$.

Preparative Chiral HPLC: Separation of the Enantiomers of 4. A hexane solution of ( \pm )-4 was prepared (290 $\mathrm{mg}$ in $18 \mathrm{~mL}$ of hexanes) and subjected to chiral preparative HPLC separation using the following conditions: Daicel Chiralpak OD (semipreparative column), hexanes: $i \mathrm{PrOH}=90: 10,2.0 \mathrm{~mL} / \mathrm{min}, 300 \mathrm{~nm}, 0.5 \mathrm{~mL} /$ injection, ((R) isomer: 9.8-11.5 $\mathrm{min},(\mathrm{S})$ isomer: 12.0-14.5 $\mathrm{min})$. The separated enantiomers of 4 were re-purified by flash chromatography (pentane $/ \mathrm{Et}_{2} \mathrm{O}$ ) under an inert atmosphere.

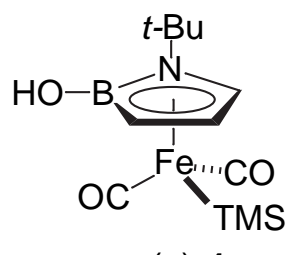

$$
(-)-4
$$

The separation procedure furnished the (-) enantiomer in $45 \%$ isolated yield as a yellow solid. The optical purity was determined to be $99 \%$ by analytical HPLC (Daicel Chiralpak OD, hexanes:i- $\mathrm{PrOH}=95: 5,1.0 \mathrm{~mL} / \mathrm{min},(\mathrm{R})$ isomer: $5.6 \mathrm{~min})$. Crystals suitable for X-ray analysis were obtained from a slowly evaporating, cold $\left(-30{ }^{\circ} \mathrm{C}\right)$ pentane/benzene solution. Thus, the absolute configuration of (-)-4 is as illustrated above.

$[\alpha]_{\mathrm{D}}^{20}=-30^{\circ}\left(\mathrm{c}=0.33, \mathrm{CH}_{2} \mathrm{Cl}_{2} ; 99 \%\right.$ ee $)$.

$\operatorname{mp} 43-44{ }^{\circ} \mathrm{C}$.

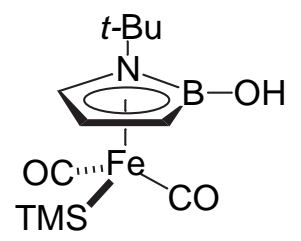

$(+)-4$

The separation procedure furnished the (+) enantiomer in $42 \%$ isolated yield as a yellow solid. The optical purity was determined to be $99 \%$ by analytical HPLC (Daicel Chiralpak OD, hexanes:i-PrOH = 95:5, $1.0 \mathrm{~mL} / \mathrm{min}$, (S) isomer: $7.2 \mathrm{~min}$ ). 


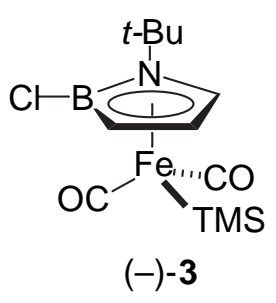

In a glove box, a 100-mL Schlenk tube was charged with a solution of (-)-4 (120 mg, $0.371 \mathrm{mmol})$ in THF $(50.0 \mathrm{~mL})$. Oxalyl chloride $(321 \mu \mathrm{L}, 3.68 \mathrm{mmol})$ was added via syringe, followed by a catalytic amount of $N, N$-dimethylformamide $(11.4 \mu \mathrm{L}, 0.147$ $\mathrm{mmol}$ ). The reaction mixture was allowed to sit at room temperature with occasional release of the pressure. After $48 \mathrm{~h},{ }^{11} \mathrm{~B}$ NMR established that the reaction had proceeded to completion. The reaction mixture was diluted with hexanes $(35 \mathrm{~mL})$ and concentrated under vacuum. When the volume was $\sim 5 \mathrm{~mL}$, more hexanes were added $(\sim 35 \mathrm{~mL})$, and the solution was concentrated to dryness under vacuum. The resulting residue was then purified by flash chromatography (pentane), which furnished the desired product as a yellow solid $(84 \mathrm{mg}, 66 \%)$. The spectral data are identical to those reported above for the racemic product.

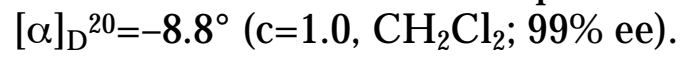

$\mathrm{mp} 102-103{ }^{\circ} \mathrm{C}$.

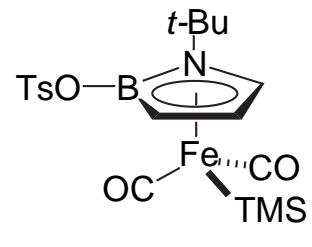

$(+)-1$

In a glove box, a vial was charged with $(-)-3(83.0 \mathrm{mg}, 0.243 \mathrm{mmol})$ and silver $p$ toluenesulfonate $(150 \mathrm{mg}, 0.535 \mathrm{mmol})$. Benzene $(2.4 \mathrm{~mL})$ was added, and the reaction mixture was stirred vigorously for $23 \mathrm{~h}$. Then, pentane was added, the reaction mixture was filtered through an acrodisc, and the filtrate was concentrated under vacuum. The residue was then purified by flash chromatography (pentane $/ \mathrm{Et}_{2} \mathrm{O}$ ), which furnished the desired product as yellow needles $(97 \mathrm{mg}, 83 \%)$.

${ }^{1} \mathrm{H}$ NMR $\left(500 \mathrm{MHz}, \mathrm{CD}_{2} \mathrm{Cl}_{2}\right): \delta 7.88\left(\mathrm{~d},{ }^{3} \mathrm{~J}_{\mathrm{HH}}=7.0 \mathrm{~Hz}, 2 \mathrm{H}\right), 7.38\left(\mathrm{~d},{ }^{3} \mathrm{~J}_{\mathrm{HH}}=8.0 \mathrm{~Hz}, 2 \mathrm{H}\right)$, $5.53(\mathrm{~s}, 1 \mathrm{H}), 4.70\left(\mathrm{~d},{ }^{3} J_{\mathrm{HH}}=4.5 \mathrm{~Hz}, 1 \mathrm{H}\right), 3.21\left(\mathrm{~d},{ }^{3} J_{\mathrm{HH}}=5.0 \mathrm{~Hz}, 1 \mathrm{H}\right), 2.44(\mathrm{~s}, 3 \mathrm{H}), 1.30(\mathrm{~s}$, 9H), $0.22(\mathrm{~s}, 9 \mathrm{H})$.

${ }^{13} \mathrm{C}$ NMR $\left(125 \mathrm{MHz}, \mathrm{CD}_{2} \mathrm{Cl}_{2}\right): \delta 214.4,214.2,145.8,135.3,130.5,128.1,89.2,83.2,62.8$ (br), 56.8, 30.9, 22.1, 7.7.

${ }^{11} \mathrm{~B}$ NMR $\left(160 \mathrm{MHz}, \mathrm{CD}_{2} \mathrm{Cl}_{2}\right): \delta 18.6$.

FTIR (thin film) 3189, 2981, 2947, 2889, 1986, 1926, 1600, 1434, 1359, 1278, 1240, 1189, $1178,1098,1090 \mathrm{~cm}^{-1}$.

HRMS (EI) calcd for $\mathrm{C}_{19} \mathrm{H}_{28} \mathrm{BFeNO}_{5} \mathrm{SSi}\left(\mathrm{M}^{+}\right)$477.0894, found 477.0906.

$[\alpha]_{D^{20}=+67^{\circ}}\left(\mathrm{c}=0.41, \mathrm{CH}_{2} \mathrm{Cl}_{2} ; 99 \%\right.$ ee $)$.

mp $90-91{ }^{\circ} \mathrm{C}$. 


\section{Synthesis of Silyl Ketene Acetals}<smiles>COC(OC)=C(C)C</smiles>

A round-bottomed flask was charged with a solution of diisopropylamine $(1.98 \mathrm{~g}$, $19.6 \mathrm{mmol})$ in THF $(45 \mathrm{~mL})$ and then cooled to $0{ }^{\circ} \mathrm{C} . n$-BuLi $(2.8 \mathrm{M}$ in hexanes; $7.0 \mathrm{~mL}$, $20 \mathrm{mmol}$ ) was added to this solution dropwise at $0{ }^{\circ} \mathrm{C}$, and the resulting mixture was stirred for $20 \mathrm{~min}$. The solution was then cooled to $-78^{\circ} \mathrm{C}$, and DMPU $(9.5 \mathrm{~mL})$ and methyl isobutyrate $(2.25 \mathrm{~mL}, 19.6 \mathrm{mmol})$ were added. After $30 \mathrm{~min}$ of stirring at -78 ${ }^{\circ} \mathrm{C}$, a solution of triisopropylsilyl triflate $(6.0 \mathrm{~g}, 20 \mathrm{mmol})$ in THF $(5 \mathrm{~mL})$ was added . The mixture was stirred for $30 \mathrm{~min}$ at $-78^{\circ} \mathrm{C}$, then warmed to room temperature and stirred for $30 \mathrm{~min}$. The reaction was quenched with a mixture of hexanes/water. The organic layer was washed with brine, dried over sodium sulfate, and concentrated under vacuum. The crude product was fractionally distilled under reduced pressure to furnish the desired product as a colorless liquid. The product was further purified by flash chromatography (pentane) $(2.7 \mathrm{~g}, 53 \%)$.

${ }^{1} \mathrm{H}$ NMR (500 MHz, $\left.\mathrm{CD}_{2} \mathrm{Cl}_{2}\right): \delta 3.53(\mathrm{~s}, 3 \mathrm{H}), 1.55$ (apparent s, 6H), 1.20-1.08 (m, 21H).

${ }^{13} \mathrm{C}$ NMR $\left(125 \mathrm{MHz}, \mathrm{CD}_{2} \mathrm{Cl}_{2}\right): \delta 151.4,91.3,58.6,18.2,17.4,16.6,13.3$.

FTIR (thin film) 2946, 2867, 1703, 1465, 1256, 1200, 1183, $1142 \mathrm{~cm}^{-1}$.

bp $62-65^{\circ} \mathrm{C}$ (300 mtorr).<smiles>C=C([SnH3])[Se]C(=C)[Se][Se]</smiles>

214287-10-2. This compound was prepared according to a literature procedure. ${ }^{2}$

${ }^{1} \mathrm{H}$ NMR $\left(500 \mathrm{MHz}, \mathrm{C}_{6} \mathrm{D}_{6}\right): \delta 4.86(\mathrm{~s}, 1 \mathrm{H}), 4.71(\mathrm{~s}, 1 \mathrm{H}), 1.42(\mathrm{~s}, 9 \mathrm{H}), 1.18-1.12(\mathrm{~m}, 21 \mathrm{H})$. FTIR (thin film) 2946, 2895, 2868, 1593, 1464, 1384, 1364, 1189, 1151, 1014, $883 \mathrm{~cm}^{-1}$. HRMS (ESI) calcd for $\mathrm{C}_{15} \mathrm{H}_{32} \mathrm{NaSSiO}\left(\mathrm{M}^{+}+\mathrm{Na}\right) 311.1835$, found 311.1831 . 


\section{Mukaiyama Aldol Additions (Table 1)}

Table 1, entry 1. In a glove box, a vial was charged with a solution of (+)-1 (46.0 mg, $0.0960 \mathrm{mmol})$ in $\mathrm{CD}_{2} \mathrm{Cl}_{2}(1.0 \mathrm{~mL})$. 4-Methoxybenzaldehyde $(24.0 \mu \mathrm{L}, 0.192 \mathrm{mmol})$ was added via a syringe at room temperature, followed by the silyl ketene acetal (54.7 mg, $0.212 \mathrm{mmol}$ ). The reaction was monitored by ${ }^{1} \mathrm{H}$ NMR spectroscopy. After seven days, the reaction mixture was concentrated under vacuum. The resulting residue was purified by flash chromatography (pentane $/ \mathrm{Et}_{2} \mathrm{O}$ ), which furnished the desired product as a yellow oil (39 $\mathrm{mg}, 74 \%)$. ${ }^{1} \mathrm{H}$ NMR indicated a de $>95 \%$.

${ }^{1} \mathrm{H}$ NMR $\left(500 \mathrm{MHz}, \mathrm{CD}_{2} \mathrm{Cl}_{2}\right): \delta 7.27\left(\mathrm{~d},{ }^{3} J_{\mathrm{HH}}=8.5 \mathrm{~Hz}, 2 \mathrm{H}\right), 6.87\left(\mathrm{~d},{ }^{3} J_{\mathrm{HH}}=8.5 \mathrm{~Hz}, 2 \mathrm{H}\right)$, $5.48(\mathrm{~s}, 1 \mathrm{H}), 5.20(\mathrm{~s}, 1 \mathrm{H}), 4.57\left(\mathrm{~d},{ }^{3} J_{\mathrm{HH}}=5.0 \mathrm{~Hz}, 1 \mathrm{H}\right), 3.79(\mathrm{~s}, 3 \mathrm{H}), 3.57(\mathrm{~s}, 3 \mathrm{H}), 2.70\left(\mathrm{~d},{ }^{3} J_{\mathrm{HH}}\right.$ $=5.0 \mathrm{~Hz}, 1 \mathrm{H}), 1.37(\mathrm{~s}, 9 \mathrm{H}), 1.16(\mathrm{~s}, 3 \mathrm{H}), 1.01(\mathrm{~s}, 3 \mathrm{H}), 0.06(\mathrm{~s}, 9 \mathrm{H})$.

${ }^{13} \mathrm{C}$ NMR $\left(125 \mathrm{MHz}, \mathrm{CD}_{2} \mathrm{Cl}_{2}\right): \delta 215.1,214.8,177.1,159.9,130.7,129.9,113.5,88.1,84.8$, 82.5, 57.2 (br), 55.7, 55.5, 52.1, 49.2, 30.5, 22.7, 19.4, 7.2.

${ }^{11} \mathrm{~B}$ NMR $\left(160 \mathrm{MHz}, \mathrm{CD}_{2} \mathrm{Cl}_{2}\right): \delta 22.0$.

FTIR (thin film) 2949, 1978, 1917, 1736, 1614, 1514, 1435, 1405, 1366, 1249, $1133 \mathrm{~cm}^{-1}$. HRMS (EI) calcd for $\mathrm{C}_{25} \mathrm{H}_{38} \mathrm{BFeNO}{ }_{6} \mathrm{Si}\left(\mathrm{M}^{+}\right)$543.1905, found 543.1921.

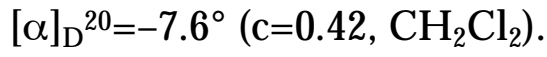

Table 1, entry 2. In a glove box, a vial was charged with a solution of $(+)-\mathbf{1}(44.0 \mathrm{mg}$, $0.0920 \mathrm{mmol})$ in $\mathrm{CD}_{2} \mathrm{Cl}_{2}(1.0 \mathrm{~mL})$. 4-Dimethlylaminobenzaldehyde $(27.5 \mathrm{mg}$, 0.184 $\mathrm{mmol}$ ) was added at room temperature, followed by the silyl ketene acetal (52.0 mg, $0.200 \mathrm{mmol}$ ). The reaction was monitored by ${ }^{1} \mathrm{H}$ NMR spectroscopy. After three days, the reaction mixture was concentrated under vacuum. The resulting residue was purified by flash chromatography (pentane $/ \mathrm{Et}_{2} \mathrm{O}$ ), which furnished the desired product as a yellow solid $(36 \mathrm{mg}, 70 \%)$. ${ }^{1} \mathrm{H}$ NMR indicated a de $>95 \%$.

${ }^{1} \mathrm{H}$ NMR $\left(500 \mathrm{MHz}, \mathrm{CD}_{2} \mathrm{Cl}_{2}\right): \delta 7.19\left(\mathrm{~d},{ }^{3} \mathrm{~J}_{\mathrm{HH}}=6.5 \mathrm{~Hz}, 2 \mathrm{H}\right), 6.69\left(\mathrm{~d},{ }^{3} \mathrm{~J}_{\mathrm{HH}}=6.5 \mathrm{~Hz}, 2 \mathrm{H}\right)$, $5.48(\mathrm{~s}, 1 \mathrm{H}), 5.15(\mathrm{~s}, 1 \mathrm{H}), 4.56\left(\mathrm{~d},{ }^{3} J_{\mathrm{HH}}=5.0 \mathrm{~Hz}, 1 \mathrm{H}\right), 3.57(\mathrm{~s}, 3 \mathrm{H}), 2.93(\mathrm{~s}, 6 \mathrm{H}), 2.69\left(\mathrm{~d},{ }^{3} J_{\mathrm{HH}}\right.$ $=5.0 \mathrm{~Hz}, 1 \mathrm{H}), 1.37(\mathrm{~s}, 9 \mathrm{H}), 1.16(\mathrm{~s}, 3 \mathrm{H}), 1.01(\mathrm{~s}, 3 \mathrm{H}), 0.06(\mathrm{~s}, 9 \mathrm{H})$.

${ }^{13} \mathrm{C}$ NMR $\left(125 \mathrm{MHz}, \mathrm{CD}_{2} \mathrm{Cl}_{2}\right): \delta 215.2,214.8,177.3,150.9,129.5,126.2,112.0,88.1,85.1$, 82.4, 57.4 (br), 55.5, 52.0, 49.4, 40.9, 30.4, 22.7, 19.4, 7.2.

${ }^{11} \mathrm{~B}$ NMR $\left(160 \mathrm{MHz}, \mathrm{CD}_{2} \mathrm{Cl}_{2}\right): \delta 21.9$.

FTIR (thin film) 2980, 2947, 2808, 1972, 1909, 1735, 1616, 1525, 1436, 1405, 1394, 1367, $1253,1134,1045 \mathrm{~cm}^{-1}$.

HRMS (EI) calcd for $\mathrm{C}_{26} \mathrm{H}_{41} \mathrm{BFeN}_{2} \mathrm{O}_{5} \mathrm{Si}\left(\mathrm{M}^{+}\right)$556.2222, found 556.2216.

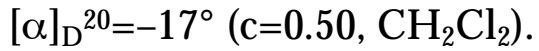

$\mathrm{mp} 120-121^{\circ} \mathrm{C}$.

Table 1, entry 3. In a glove box, a vial was charged with a solution of $( \pm)-\mathbf{1}(30.0 \mathrm{mg}$, $0.0600 \mathrm{mmol})$ in $\mathrm{CD}_{2} \mathrm{Cl}_{2}(0.7 \mathrm{~mL})$. Indole-3-carboxaldehyde $(20.0 \mathrm{mg}, 0.125 \mathrm{mmol})$ was added at room temperature, followed by the silyl ketene acetal $(35.7 \mathrm{mg}, 0.138 \mathrm{mmol})$. The reaction was monitored by ${ }^{1} \mathrm{H}$ NMR spectroscopy. After three days, the reaction mixture was concentrated under vacuum. The resulting residue was purified by flash chromatography (pentane $/ \mathrm{Et}_{2} \mathrm{O}$ ), which furnished the desired product as a yellow solid (30 mg, 85\%). ${ }^{1} \mathrm{H}$ NMR indicated a de $>95 \%$.

Crystals suitable for X-ray analysis were grown from a concentrated pentane/benzene solution at $-30^{\circ} \mathrm{C}$. 
${ }^{1} \mathrm{H}$ NMR $\left(500 \mathrm{MHz}, \mathrm{CD}_{2} \mathrm{Cl}_{2}\right): \delta 7.67\left(\mathrm{~d},{ }^{3} J_{\mathrm{HH}}=8.0 \mathrm{~Hz}, 1 \mathrm{H}\right), 7.32\left(\mathrm{~d},{ }^{3} J_{\mathrm{HH}}=8.5 \mathrm{~Hz}, 1 \mathrm{H}\right)$, 7.21-7.17 (m, 2H), 7.10 $\left(\mathrm{t},{ }^{3} \mathrm{~J}_{\mathrm{HH}}=7.3 \mathrm{~Hz}, 1 \mathrm{H}\right), 5.67(\mathrm{~s}, 1 \mathrm{H}), 5.46(\mathrm{~s}, 1 \mathrm{H}), 4.48\left(\mathrm{~d},{ }^{3} J_{\mathrm{HH}}=5.5\right.$ $\mathrm{Hz}, 1 \mathrm{H}), 3.77(\mathrm{~s}, 3 \mathrm{H}), 3.58(\mathrm{~s}, 3 \mathrm{H}), 2.53\left(\mathrm{~d},{ }^{3} J_{\mathrm{HH}}=5.5 \mathrm{~Hz}, 1 \mathrm{H}\right), 1.35(\mathrm{~s}, 9 \mathrm{H}), 1.34(\mathrm{~s}, 3 \mathrm{H})$, $1.08(\mathrm{~s}, 3 \mathrm{H}),-0.13(\mathrm{~s}, 9 \mathrm{H})$.

${ }^{13} \mathrm{C}$ NMR $\left(125 \mathrm{MHz}, \mathrm{CD}_{2} \mathrm{Cl}_{2}\right): \delta 215.2,215.1,177.5,137.0,129.9,128.6,121.6,119.6$, 119.5, 111.7, 109.8, 88.1, 82.6, 78.8, 57.8 (br), 55.4, 52.1, 49.6, 33.3, 30.4, 23.0, 19.3, 7.1.

${ }^{11} \mathrm{~B}$ NMR $\left(160 \mathrm{MHz}, \mathrm{CD}_{2} \mathrm{Cl}_{2}\right): \delta 22.2$.

FTIR (thin film) 2947, 2886, 1974, 1914, 1735, 1651, 1549, 1470, 1434, 1411, 1385, 1366, $1256,1239,1134 \mathrm{~cm}^{-1}$.

HRMS (ESI) calcd for $\mathrm{C}_{27} \mathrm{H}_{39} \mathrm{BFeN}_{2} \mathrm{NaO}_{5} \mathrm{Si}\left(\mathrm{M}^{+}+\mathrm{Na}\right)$ 589.1963, found 589.1935. mp $147-148{ }^{\circ} \mathrm{C}$.

Table 1, entry 4. In a glove box, a vial was charged with a solution of $( \pm)-\mathbf{1}(30.0 \mathrm{mg}$, $0.0600 \mathrm{mmol})$ in $\mathrm{CD}_{3} \mathrm{CN}(0.6 \mathrm{~mL})$. Indole-3-carboxaldehyde $(20.0 \mathrm{mg}, 0.125 \mathrm{mmol})$ was added at room temperature, followed by the nucleophile $(38.5 \mathrm{mg}, 0.133 \mathrm{mmol})$. After $20 \mathrm{~h},{ }^{1} \mathrm{H}$ NMR spectroscopy showed that the reaction was complete $(74 \% \mathrm{de})$. The desired product precipitated out of solution upon concentration under vacuum. The yellow precipitate was washed thoroughly with pentane and then with cold $\mathrm{Et}_{2} \mathrm{O}$ to furnish the aldol adduct as yellow crystals ( $26 \mathrm{mg}, 72 \%$; de $>95 \%$ according to ${ }^{1} \mathrm{H} \mathrm{NMR}$ ).

Crystals suitable for $\mathrm{X}$-ray analysis were grown from a concentrated pentane/benzene $/ \mathrm{CH}_{2} \mathrm{Cl}_{2}$ solution at $-30{ }^{\circ} \mathrm{C}$.

${ }^{1} \mathrm{H}$ NMR $\left(500 \mathrm{MHz}, \mathrm{CD}_{2} \mathrm{Cl}_{2}\right): \delta 7.71\left(\mathrm{~d},{ }^{3} \mathrm{~J}_{\mathrm{HH}}=8.0 \mathrm{~Hz}, 1 \mathrm{H}\right), 7.31\left(\mathrm{~d},{ }^{3} J_{\mathrm{HH}}=8.5 \mathrm{~Hz}, 1 \mathrm{H}\right)$, $7.21\left(\mathrm{td},{ }^{3} J_{\mathrm{HH}}=8.0 \mathrm{~Hz}, 1.0 \mathrm{~Hz}, 1 \mathrm{H}\right), 7.14(\mathrm{~s}, 1 \mathrm{H}), 7.10\left(\mathrm{td},{ }^{3} J_{\mathrm{HH}}=8.0 \mathrm{~Hz}, 1.0 \mathrm{~Hz}, 1 \mathrm{H}\right), 5.70$ $\left(\mathrm{dd},{ }^{3} J_{\mathrm{HH}}=9.5 \mathrm{~Hz}, 4.0 \mathrm{~Hz}, 1 \mathrm{H}\right), 5.49(\mathrm{~s}, 1 \mathrm{H}), 4.58\left(\mathrm{~d},{ }^{3} \mathrm{~J}_{\mathrm{HH}}=5.0 \mathrm{~Hz}, 1 \mathrm{H}\right), 3.75(\mathrm{~s}, 3 \mathrm{H}), 3.33$ $\left(\mathrm{dd},{ }^{3} J_{\mathrm{HH}}=15.0 \mathrm{~Hz}, 9.5 \mathrm{~Hz}, 1 \mathrm{H}\right), 2.92\left(\mathrm{dd},{ }^{3} J_{\mathrm{HH}}=15.0 \mathrm{~Hz}, 4.0 \mathrm{~Hz}, 1 \mathrm{H}\right), 2.83\left(\mathrm{~d},{ }^{3} J_{\mathrm{HH}}=5.0\right.$ $\mathrm{Hz}, 1 \mathrm{H}), 1.40(\mathrm{~s}, 9 \mathrm{H}), 1.34(\mathrm{~s}, 9 \mathrm{H}), 0.05(\mathrm{~s}, 9 \mathrm{H})$.

${ }^{13} \mathrm{C}$ NMR $\left(125 \mathrm{MHz}, \mathrm{CD}_{2} \mathrm{Cl}_{2}\right): \delta 215.10,215.06,197.9,138.0,128.0,126.5,122.0,120.0$, $119.5,115.1,110.0,88.2,82.6,71.3,57.6(\mathrm{br}), 55.5,53.6,48.5,33.2,30.5,30.0,7.2$.

${ }^{11} \mathrm{~B}$ NMR $\left(160 \mathrm{MHz}, \mathrm{CD}_{2} \mathrm{Cl}_{2}\right): \delta 21.9$.

FTIR (thin film) 2962, 1976, 1914, 1683, 1557, 1408, 1392, 1366, 1239, $1209 \mathrm{~cm}^{-1}$. HRMS (ESI) calcd for $\mathrm{C}_{28} \mathrm{H}_{41} \mathrm{BFeN}_{2} \mathrm{NaO}_{4} \mathrm{SSi}\left(\mathrm{M}^{+}+\mathrm{Na}\right)$ 619.1981, found 619.1902. mp $180-183{ }^{\circ} \mathrm{C}$ (decomposition). 


\section{Hydrolysis of the Boron Aldolate (e.g., eq 3)}

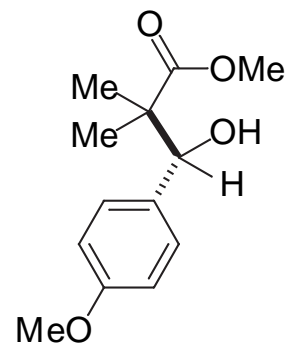

Eight drops of a 1.0 M TBAF solution in THF were added to a solution of the boron aldolate $(31.0 \mathrm{mg}, 0.0570 \mathrm{mmol})$ in wet THF $(1.5 \mathrm{~mL}$, along with 2 drops of water). After $4 \mathrm{~h}$ of stirring, a mixture of $\mathrm{Et}_{2} \mathrm{O}$ /water was added. The aqueous phase was extracted three times with $\mathrm{Et}_{2} \mathrm{O}$. The combined organic layers were dried over $\mathrm{Na}_{2} \mathrm{SO}_{4}$, filtered, and concentrated under reduced pressure. The resulting crude material was purified by flash chromatography (pentane $/ \mathrm{Et}_{2} \mathrm{O}=1: 1$ ) to furnish the desired product as a colorless oil $(11.8 \mathrm{mg}, 87 \%)$. The spectral data are identical to those reported in the literature. ${ }^{3}$

\section{$[\alpha]_{D^{20}=-22^{\circ}}\left(\mathrm{c}=1.0, \mathrm{CH}_{2} \mathrm{Cl}_{2}\right)$.}

The enantiomeric excess was determined to be $96 \%$ by HPLC analysis (Daicel Chiralpak OD, hexane:i-PrOH = 95:5, $0.8 \mathrm{~mL} / \mathrm{min}$; (S) isomer (minor): $15.7 \mathrm{~min},(\mathrm{R})$ isomer (major): $19.4 \mathrm{~min}$ ). The assignment of absolute configuration was made by comparison with the reported HPLC retention times: (S) isomer: $15.5 \mathrm{~min},(\mathrm{R})$ isomer: $19.1 \min ^{3}$

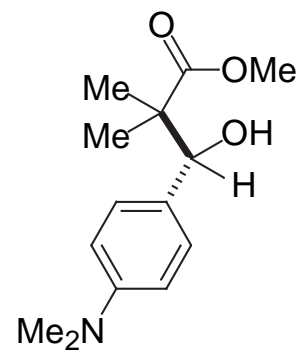

Product of Table 1, entry 2. Eight drops of a 1.0 M TBAF solution in THF were added to a solution of the boron aldolate $(28.0 \mathrm{mg}, 0.0500 \mathrm{mmol})$ in wet THF $(1.5 \mathrm{~mL}$, along with 2 drops of water). After $4 \mathrm{~h}$ of stirring, a mixture of $\mathrm{Et}_{2} \mathrm{O}$ /water was added. The aqueous phase was extracted three times with $\mathrm{Et}_{2} \mathrm{O}$. The combined organic layers were dried over $\mathrm{Na}_{2} \mathrm{SO}_{4}$, filtered, and concentrated under reduced pressure. The resulting crude material was purified by flash chromatography (pentane $/ \mathrm{Et}_{2} \mathrm{O}=1: 1$ ) to furnish the desired product as a white solid $(12.5 \mathrm{mg}, 99 \%)$.

${ }^{1} \mathrm{H} \mathrm{NMR}\left(500 \mathrm{MHz}, \mathrm{CD}_{2} \mathrm{Cl}_{2}\right): \delta 7.13\left(\mathrm{~d},{ }^{3} \mathrm{~J}_{\mathrm{HH}}=8.5 \mathrm{~Hz}, 2 \mathrm{H}\right), 6.67\left(\mathrm{~d},{ }^{3} \mathrm{~J}_{\mathrm{HH}}=9.0 \mathrm{~Hz}, 2 \mathrm{H}\right)$, $4.77(\mathrm{~s}, 1 \mathrm{H}), 3.68(\mathrm{~s}, 3 \mathrm{H}), 2.93(\mathrm{~s}, 6 \mathrm{H}), 2.78(\mathrm{~s}, 1 \mathrm{H}), 1.11(\mathrm{~s}, 3 \mathrm{H}), 1.06(\mathrm{~s}, 3 \mathrm{H})$.

${ }^{13} \mathrm{C}$ NMR $\left(125 \mathrm{MHz}, \mathrm{CD}_{2} \mathrm{Cl}_{2}\right): \delta 178.5,150.8,128.8,128.4,112.1,79.0,52.3,48.4,40.8$, 23.2, 19.6.

FTIR (thin film) 3471, 2988, 2953, 2888, 2806, 1705, 1693, 1614, 1524, 1471, 1444, 1430, 
$1364,1339,1273,1230,1188,1154,1054 \mathrm{~cm}^{-1}$.

HRMS (EI) calcd for $\mathrm{C}_{14} \mathrm{H}_{21} \mathrm{NO}_{3}\left(\mathrm{M}^{+}\right)$251.1516, found 251.1510.

$[\alpha]_{D^{20}}=-32^{\circ}\left(\mathrm{c}=1.0, \mathrm{CH}_{2} \mathrm{Cl}_{2}\right)$.

$\mathrm{mp} 110-112{ }^{\circ} \mathrm{C}$.

The enantiomeric excess was determined to be $99 \%$ by HPLC analysis (Daicel Chiralpak OD, hexane:i-PrOH = 95:5, $1.0 \mathrm{~mL} / \mathrm{min}$; (S) isomer (minor): $14.9 \mathrm{~min}$, (R) isomer (major): $17.4 \mathrm{~min}$ ).

The assignment of absolute configuration was made by analogy to our assignments for entries 1,3 , and 4 . 


\section{X-ray Crystal Structures}

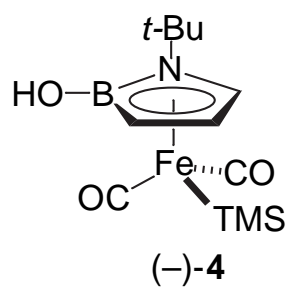

A yellow pentane/benzene solution was prepared. Crystals suitable for X-ray structural analysis were obtained by solvent evaporation at $-30{ }^{\circ} \mathrm{C}$.

A yellow needle of dimensions $0.29 \times 0.14 \times 0.1 \mathrm{~mm}^{3}$ was mounted under STP and transferred to a Bruker AXS/CCD three-circle diffractometer equipped with a cold stream of $\mathrm{N}_{2}$ gas. An initial unit cell was determined with monochromated Mo $\mathrm{K}_{\alpha}$ radiation $(\lambda=0.71073 \AA$ ). The cell thus determined was orthorhombic.

A hemisphere of data was then collected using $\omega$ scans of $0.30^{\circ}$ and $30-\mathrm{s}$ frames. The raw data frames were integrated using the Bruker program SAINT+ for NT version 6.01. The data that were collected (7849 total reflections, 4972 unique, $R_{\text {int }}=0.0815$ ) had the following Miller index ranges: -7 to 4 in $h,-15$ to 18 in $k$, and -27 to 35 in 1 . No absorption correction was performed.

All aspects of the solution and refinement were handled by SHELXTL NT version 5.10. The structure was solved by direct methods in the chiral orthorhombic space group P2 ${ }_{1}{ }_{1} 21, a=6.760(4) \AA ; b=16.787(11) \AA ; c=32.061(16) \AA ; \alpha=90^{\circ} ; \beta=90^{\circ} ; \gamma=90^{\circ}$, and refined using standard difference Fourier techniques. Final, full-matrix leastsquares refinement (4972 data for 357 parameters) on F2 yielded residuals of R1 and wR2 of 0.0849 and 0.2126 for data $\mathrm{I}>2 \sigma(\mathrm{I})$, and 0.1307 and 0.2508 , respectively, for all data. During the final refinement all non-hydrogen atoms were treated anisotropically. Hydrogen atoms were included in calculated positions and refined isotropically on a riding model. No extinction coefficient was used in the refinement. Residual electron density amounted to a maximum of $1.955 \mathrm{e} / \AA^{-3}$ and a minimum of $-0.819 \mathrm{e} / \AA^{-3}$.

The absolute structure (Flack) parameter for the correct enantiomer is -0.08(6). The structure was also inverted and refined in order to confirm the initial assignment of absolute stereochemistry.

Tables 1-6 provide the full crystallographic data for the X-ray structure. 


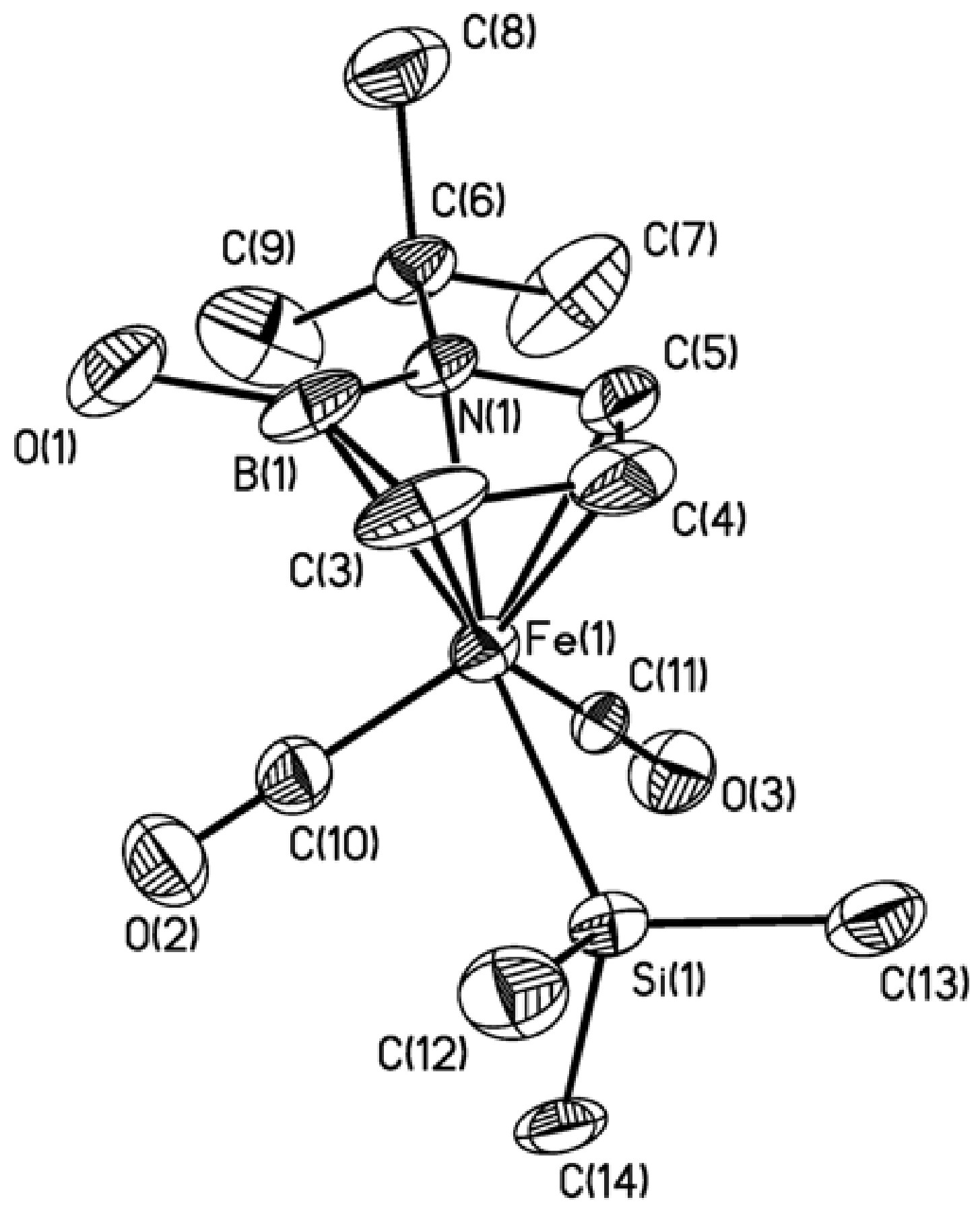


Table 1. Crystal data and structure refinement for 03199SL0.

Identification code

Empirical formula

Formula weight

Temperature

Wavelength

Crystal system

Space group

Unit cell dimensions

Volume

Z

Density (calculated)

Absorption coefficient

$\mathrm{F}(000)$

Crystal size

Theta range for data collection

Index ranges

Reflections collected

Independent reflections

Completeness to theta $=23.28^{\circ}$

Refinement method

Data / restraints / parameters

Goodness-of-fit on $\mathrm{F}^{2}$

Final $\mathrm{R}$ indices [I>2sigma(I)]

$\mathrm{R}$ indices (all data)

Absolute structure parameter

Largest diff. peak and hole 03199s10

C14 H26 B Fe N O3.50 Si

359.11

193(2) K

$0.71073 \AA$

Orthorhombic

P2(1)2(1)2(1)

$\mathrm{a}=6.760(4) \AA$

$\mathrm{a}=90^{\circ}$.

$\mathrm{b}=16.787(11) \AA$

$\mathrm{b}=90^{\circ}$.

$\mathrm{c}=32.061(16) \AA$

$\mathrm{g}=90^{\circ}$.

3638(4) $\AA^{3}$

8

$1.311 \mathrm{Mg} / \mathrm{m}^{3}$

$0.905 \mathrm{~mm}^{-1}$

1520

$0.29 \times 0.14 \times 0.1 \mathrm{~mm}^{3}$

1.27 to $23.28^{\circ}$.

$-7<=\mathrm{h}<=4,-15<=\mathrm{k}<=18,-27<=\mathrm{l}<=35$

7849

$4972[\mathrm{R}(\mathrm{int})=0.0815]$

$97.1 \%$

Full-matrix least-squares on $\mathrm{F}^{2}$

4972 / 0 / 400

0.945

$\mathrm{R} 1=0.0677, \mathrm{wR} 2=0.1419$

$\mathrm{R} 1=0.1141, \mathrm{wR} 2=0.1691$

$-0.08(4)$

0.411 and -0.676 e. $\AA^{-3}$ 
Table 2. Atomic coordinates ( x 104) and equivalent isotropic displacement parameters $\left(\AA^{2} \times 10^{3}\right)$ for 03199SL0. $U(e q)$ is defined as one third of the trace of the orthogonalized $U$ ij tensor.

\begin{tabular}{|c|c|c|c|c|}
\hline & $x$ & $\mathrm{y}$ & $\mathrm{z}$ & $\mathrm{U}(\mathrm{eq})$ \\
\hline $\mathrm{Fe}(1)$ & $8262(2)$ & $3461(1)$ & $8314(1)$ & $36(1)$ \\
\hline $\mathrm{Fe}(2)$ & $3822(2)$ & $3710(1)$ & $5937(1)$ & $33(1)$ \\
\hline $\operatorname{Si}(1)$ & $7578(4)$ & $4822(2)$ & $8347(1)$ & $41(1)$ \\
\hline $\operatorname{Si}(2)$ & $5205(4)$ & $3175(2)$ & $6540(1)$ & $39(1)$ \\
\hline $\mathrm{O}(3)$ & $10900(12)$ & $3984(5)$ & $7648(2)$ & $63(2)$ \\
\hline $\mathrm{N}(1)$ & $7633(12)$ & $2231(5)$ & $8230(3)$ & $41(2)$ \\
\hline $\mathrm{O}(5)$ & $536(11)$ & $4163(5)$ & $6470(2)$ & $61(2)$ \\
\hline$C(26)$ & $2751(15)$ & $4068(6)$ & $4941(3)$ & $42(3)$ \\
\hline$C(11)$ & 9853(16) & $3763(6)$ & $7912(3)$ & $39(3)$ \\
\hline$C(10)$ & 9909(17) & $3636(7)$ & $8710(3)$ & $49(3)$ \\
\hline $\mathrm{N}(2)$ & $3883(12)$ & $4248(4)$ & $5341(2)$ & $32(2)$ \\
\hline$C(30)$ & $1858(16)$ & $3974(6)$ & $6260(3)$ & $36(3)$ \\
\hline$C(24)$ & $6500(16)$ & $4231(6)$ & $5794(3)$ & $46(3)$ \\
\hline $\mathrm{O}(2)$ & 11012(13) & $3758(5)$ & $8983(2)$ & $73(2)$ \\
\hline$C(32)$ & $6077(16)$ & $3980(6)$ & $6910(3)$ & $50(3)$ \\
\hline $\mathrm{O}(6)$ & $2784(12)$ & $2055(4)$ & $5797(2)$ & $58(2)$ \\
\hline$C(23)$ & $5265(15)$ & $4841(6)$ & $5943(3)$ & $45(3)$ \\
\hline $\mathrm{B}(2)$ & $3620(19)$ & $4927(7)$ & $5626(4)$ & $43(3)$ \\
\hline$C(14)$ & $9887(16)$ & $5456(6)$ & $8385(4)$ & $56(3)$ \\
\hline$C(25)$ & $5687(14)$ & $3864(6)$ & $5450(3)$ & $38(3)$ \\
\hline$C(33)$ & $3359(18)$ & $2583(7)$ & $6854(3)$ & $57(3)$ \\
\hline$C(5)$ & 6479(19) & $2716(7)$ & $7967(4)$ & $57(3)$ \\
\hline$C(6)$ & $8903(18)$ & $1573(6)$ & $8051(3)$ & $48(3)$ \\
\hline$C(28)$ & $3321(18)$ & $3247(6)$ & $4773(3)$ & $58(3)$ \\
\hline$C(8)$ & $7640(20)$ & $794(7)$ & $8049(4)$ & $84(4)$ \\
\hline$C(13)$ & 6196(19) & $5210(6)$ & $7882(4)$ & $68(4)$ \\
\hline$C(12)$ & 6014(19) & $5086(7)$ & $8794(3)$ & $67(3)$ \\
\hline$C(3)$ & $5655(18)$ & $3093(6)$ & $8631(5)$ & $72(4)$ \\
\hline$C(27)$ & $3250(20)$ & $4695(7)$ & $4625(3)$ & $67(4)$ \\
\hline$C(31)$ & $3257(15)$ & $2719(7)$ & $5853(3)$ & $48(3)$ \\
\hline$C(4)$ & $5309(18)$ & $3200(8)$ & $8185(5)$ & $75(4)$ \\
\hline$C(34)$ & $7309(16)$ & $2477(6)$ & $6426(3)$ & $54(3)$ \\
\hline$C(7)$ & $9440(30)$ & $1766(8)$ & $7608(4)$ & 127(7) \\
\hline $\mathrm{B}(1)$ & $7000(20)$ & $2378(8)$ & $8648(5)$ & $59(4)$ \\
\hline$C(29)$ & $561(15)$ & $4068(7)$ & $5055(3)$ & $62(3)$ \\
\hline $\mathrm{O}(1)$ & $7716(15)$ & $1946(4)$ & $8993(2)$ & $81(3)$ \\
\hline$C(9)$ & 10681(18) & $1481(8)$ & $8314(5)$ & $100(5)$ \\
\hline $\mathrm{O}(4)$ & $2118(12)$ & $5475(4)$ & $5575(2)$ & $54(2)$ \\
\hline $\mathrm{O}(7)$ & $8120(50)$ & $7301(12)$ & $4754(6)$ & $283(14)$ \\
\hline$C(51)$ & $7500(90)$ & $6170(30)$ & $5270(20)$ & $510(40)$ \\
\hline$C(53)$ & $7750(80)$ & $7650(20)$ & $5160(8)$ & $340(30)$ \\
\hline$C(50)$ & 7990(100) & 6373(13) & $4827(15)$ & $480(50)$ \\
\hline
\end{tabular}


Table 3. Bond lengths $[\AA ̊]$ and angles $\left[{ }^{\circ}\right]$ for 03199SL0.

\begin{tabular}{|c|c|}
\hline $\mathrm{Fe}(1)-\mathrm{C}(10)$ & $1.713(12)$ \\
\hline $\mathrm{Fe}(1)-\mathrm{C}(11)$ & $1.754(12)$ \\
\hline $\mathrm{Fe}(1)-\mathrm{C}(5)$ & $2.062(11)$ \\
\hline $\mathrm{Fe}(1)-\mathrm{C}(4)$ & $2.085(11)$ \\
\hline $\mathrm{Fe}(1)-\mathrm{N}(1)$ & $2.124(8)$ \\
\hline $\mathrm{Fe}(1)-\mathrm{C}(3)$ & $2.127(11)$ \\
\hline $\mathrm{Fe}(1)-\mathrm{B}(1)$ & $2.275(12)$ \\
\hline $\mathrm{Fe}(1)-\mathrm{Si}(1)$ & $2.333(3)$ \\
\hline $\mathrm{Fe}(2)-\mathrm{C}(31)$ & $1.728(13)$ \\
\hline $\mathrm{Fe}(2)-\mathrm{C}(30)$ & $1.740(11)$ \\
\hline $\mathrm{Fe}(2)-\mathrm{C}(25)$ & $2.024(10)$ \\
\hline $\mathrm{Fe}(2)-\mathrm{C}(24)$ & $2.062(10)$ \\
\hline $\mathrm{Fe}(2)-\mathrm{N}(2)$ & $2.113(7)$ \\
\hline $\mathrm{Fe}(2)-\mathrm{C}(23)$ & $2.136(10)$ \\
\hline $\mathrm{Fe}(2)-\mathrm{B}(2)$ & $2.277(12)$ \\
\hline $\mathrm{Fe}(2)-\mathrm{Si}(2)$ & $2.328(3)$ \\
\hline $\operatorname{Si}(1)-C(12)$ & $1.834(11)$ \\
\hline $\operatorname{Si}(1)-C(13)$ & $1.875(11)$ \\
\hline $\operatorname{Si}(1)-C(14)$ & $1.893(10)$ \\
\hline $\operatorname{Si}(2)-C(34)$ & $1.879(10)$ \\
\hline $\operatorname{Si}(2)-C(33)$ & $1.886(11)$ \\
\hline $\operatorname{Si}(2)-C(32)$ & $1.892(10)$ \\
\hline $\mathrm{O}(3)-\mathrm{C}(11)$ & $1.163(11)$ \\
\hline $\mathrm{N}(1)-\mathrm{C}(5)$ & $1.406(13)$ \\
\hline $\mathrm{N}(1)-\mathrm{B}(1)$ & $1.430(16)$ \\
\hline $\mathrm{N}(1)-\mathrm{C}(6)$ & $1.513(13)$ \\
\hline $\mathrm{O}(5)-\mathrm{C}(30)$ & $1.164(11)$ \\
\hline$C(26)-C(27)$ & $1.500(14)$ \\
\hline C(26)-N(2) & $1.526(12)$ \\
\hline$C(26)-C(29)$ & $1.525(15)$ \\
\hline$C(26)-C(28)$ & $1.527(14)$ \\
\hline $\mathrm{C}(10)-\mathrm{O}(2)$ & $1.168(11)$ \\
\hline $\mathrm{N}(2)-\mathrm{C}(25)$ & $1.422(12)$ \\
\hline $\mathrm{N}(2)-\mathrm{B}(2)$ & 1.471(14) \\
\hline$C(24)-C(25)$ & $1.379(12)$ \\
\hline$C(24)-C(23)$ & $1.405(14)$ \\
\hline $\mathrm{O}(6)-\mathrm{C}(31)$ & $1.174(12)$ \\
\hline$C(23)-B(2)$ & $1.513(16)$ \\
\hline $\mathrm{B}(2)-\mathrm{O}(4)$ & $1.379(13)$ \\
\hline$C(5)-C(4)$ & $1.332(16)$ \\
\hline$C(6)-C(9)$ & $1.476(15)$ \\
\hline$C(6)-C(7)$ & $1.502(14)$ \\
\hline$C(6)-C(8)$ & $1.561(15)$ \\
\hline$C(3)-C(4)$ & $1.461(17)$ \\
\hline $\mathrm{C}(3)-\mathrm{B}(1)$ & 1.507(18) \\
\hline
\end{tabular}




$\begin{array}{ll}\mathrm{B}(1)-\mathrm{O}(1) & 1.408(16) \\ \mathrm{O}(7)-\mathrm{C}(53) & 1.45(2) \\ \mathrm{O}(7)-\mathrm{C}(50) & 1.58(3) \\ \mathrm{C}(51)-\mathrm{C}(50) & 1.49(7) \\ \mathrm{C}(51)-\mathrm{C}(52) & 1.42(7) \\ \mathrm{C}(53)-\mathrm{C}(52) & 1.52(4) \\ & \\ \mathrm{C}(10)-\mathrm{Fe}(1)-\mathrm{C}(11) & 95.6(5) \\ \mathrm{C}(10)-\mathrm{Fe}(1)-\mathrm{C}(5) & 152.0(5) \\ \mathrm{C}(11)-\mathrm{Fe}(1)-\mathrm{C}(5) & 97.9(5) \\ \mathrm{C}(10)-\mathrm{Fe}(1)-\mathrm{C}(4) & 143.6(6) \\ \mathrm{C}(11)-\mathrm{Fe}(1)-\mathrm{C}(4) & 120.1(6) \\ \mathrm{C}(5)-\mathrm{Fe}(1)-\mathrm{C}(4) & 37.5(5) \\ \mathrm{C}(10)-\mathrm{Fe}(1)-\mathrm{N}(1) & 113.0(4) \\ \mathrm{C}(11)-\mathrm{Fe}(1)-\mathrm{N}(1) & 108.0(4) \\ \mathrm{C}(5)-\mathrm{Fe}(1)-\mathrm{N}(1) & 39.2(4) \\ \mathrm{C}(4)-\mathrm{Fe}(1)-\mathrm{N}(1) & 65.1(4) \\ \mathrm{C}(10)-\mathrm{Fe}(1)-\mathrm{C}(3) & 103.5(6) \\ \mathrm{C}(11)-\mathrm{Fe}(1)-\mathrm{C}(3) & 160.7(6) \\ \mathrm{C}(5)-\mathrm{Fe}(1)-\mathrm{C}(3) & 66.3(5) \\ \mathrm{C}(4)-\mathrm{Fe}(1)-\mathrm{C}(3) & 40.6(5) \\ \mathrm{N}(1)-\mathrm{Fe}(1)-\mathrm{C}(3) & 67.2(4) \\ \mathrm{C}(10)-\mathrm{Fe}(1)-\mathrm{B}(1) & 91.8(5) \\ \mathrm{C}(11)-\mathrm{Fe}(1)-\mathrm{B}(1) & 143.8(5) \\ \mathrm{C}(5)-\mathrm{Fe}(1)-\mathrm{B}(1) & 63.3(5) \\ \mathrm{C}(4)-\mathrm{Fe}(1)-\mathrm{B}(1) & 64.4(6) \\ \mathrm{N}(1)-\mathrm{Fe}(1)-\mathrm{B}(1) & 37.7(4) \\ \mathrm{C}(3)-\mathrm{Fe}(1)-\mathrm{B}(1) & 39.9(5) \\ \mathrm{C}(10)-\mathrm{Fe}(1)-\mathrm{Si}(1) & 85.8(4) \\ \mathrm{C}(11)-\mathrm{Fe}(1)-\mathrm{Si}(1) & 82.7(3) \\ \mathrm{C}(5)-\mathrm{Fe}(1)-\mathrm{Si}(1) & 120.2(4) \\ \mathrm{C}(4)-\mathrm{Fe}(1)-\mathrm{Si}(1) & 91.4(4) \\ \mathrm{N}(1)-\mathrm{Fe}(1)-\mathrm{Si}(1) & 156.5(2) \\ \mathrm{C}(3)-\mathrm{Fe}(1)-\mathrm{Si}(1) & 95.6(3) \\ \mathrm{B}(1)-\mathrm{Fe}(1)-\mathrm{Si}(1) & 133.3(4) \\ \mathrm{C}(31)-\mathrm{Fe}(2)-\mathrm{C}(30) & 99.8(5) \\ \mathrm{C}(31)-\mathrm{Fe}(2)-\mathrm{C}(25) & 98.0(4) \\ \mathrm{C}(30)-\mathrm{Fe}(2)-\mathrm{C}(25) & 154.4(4) \\ \mathrm{C}(31)-\mathrm{Fe}(2)-\mathrm{C}(24) & 124.6(5) \\ \mathrm{C}(30)-\mathrm{Fe}(2)-\mathrm{C}(24) & 133.9(4) \\ \mathrm{C}(25)-\mathrm{Fe}(2)-\mathrm{C}(24) & 39.4(4) \\ \mathrm{C}(31)-\mathrm{Fe}(2)-\mathrm{N}(2) & 105.9(4) \\ \mathrm{C}(30)-\mathrm{Fe}(2)-\mathrm{N}(2) & 116.3(4) \\ \mathrm{C}(25)-\mathrm{Fe}(2)-\mathrm{N}(2) & 40.1(3) \\ \mathrm{C}(24)-\mathrm{Fe}(2)-\mathrm{N}(2) & 66.5(4) \\ \mathrm{C}(31)-\mathrm{Fe}(2)-\mathrm{C}(23) & 163.4(5) \\ \mathrm{C}(30)-\mathrm{Fe}(2)-\mathrm{C}(23) & 96.7(4)\end{array}$




$\begin{array}{ll}\mathrm{C}(25)-\mathrm{Fe}(2)-\mathrm{C}(23) & 66.9(4) \\ \mathrm{C}(24)-\mathrm{Fe}(2)-\mathrm{C}(23) & 39.1(4) \\ \mathrm{N}(2)-\mathrm{Fe}(2)-\mathrm{C}(23) & 67.5(3) \\ \mathrm{C}(31)-\mathrm{Fe}(2)-\mathrm{B}(2) & 141.4(5) \\ \mathrm{C}(30)-\mathrm{Fe}(2)-\mathrm{B}(2) & 89.2(5) \\ \mathrm{C}(25)-\mathrm{Fe}(2)-\mathrm{B}(2) & 65.5(4) \\ \mathrm{C}(24)-\mathrm{Fe}(2)-\mathrm{B}(2) & 64.9(4) \\ \mathrm{N}(2)-\mathrm{Fe}(2)-\mathrm{B}(2) & 38.9(4) \\ \mathrm{C}(23)-\mathrm{Fe}(2)-\mathrm{B}(2) & 39.9(4) \\ \mathrm{C}(31)-\mathrm{Fe}(2)-\mathrm{Si}(2) & 81.2(3) \\ \mathrm{C}(30)-\mathrm{Fe}(2)-\mathrm{Si}(2) & 84.9(3) \\ \mathrm{C}(25)-\mathrm{Fe}(2)-\mathrm{Si}(2) & 116.1(3) \\ \mathrm{C}(24)-\mathrm{Fe}(2)-\mathrm{Si}(2) & 89.7(3) \\ \mathrm{N}(2)-\mathrm{Fe}(2)-\mathrm{Si}(2) & 155.2(2) \\ \mathrm{C}(23)-\mathrm{Fe}(2)-\mathrm{Si}(2) & 98.8(3) \\ \mathrm{B}(2)-\mathrm{Fe}(2)-\mathrm{Si}(2) & 137.2(3) \\ \mathrm{C}(12)-\mathrm{Si}(1)-\mathrm{C}(13) & 104.4(6) \\ \mathrm{C}(12)-\mathrm{Si}(1)-\mathrm{C}(14) & 106.8(5) \\ \mathrm{C}(13)-\mathrm{Si}(1)-\mathrm{C}(14) & 105.5(5) \\ \mathrm{C}(12)-\mathrm{Si}(1)-\mathrm{Fe}(1) & 112.7(4) \\ \mathrm{C}(13)-\mathrm{Si}(1)-\mathrm{Fe}(1) & 113.7(4) \\ \mathrm{C}(14)-\mathrm{Si}(1)-\mathrm{Fe}(1) & 112.9(3) \\ \mathrm{C}(34)-\mathrm{Si}(2)-\mathrm{C}(33) & 106.0(5) \\ \mathrm{C}(34)-\mathrm{Si}(2)-\mathrm{C}(32) & 109.4(5) \\ \mathrm{C}(33)-\mathrm{Si}(2)-\mathrm{C}(32) & 104.4(5) \\ \mathrm{C}(34)-\mathrm{Si}(2)-\mathrm{Fe}(2) & 112.5(4) \\ \mathrm{C}(33)-\mathrm{Si}(2)-\mathrm{Fe}(2) & 112.4(4) \\ \mathrm{C}(32)-\mathrm{Si}(2)-\mathrm{Fe}(2) & 111.8(3) \\ \mathrm{C}(5)-\mathrm{N}(1)-\mathrm{B}(1) & 107.3(10) \\ \mathrm{C}(5)-\mathrm{N}(1)-\mathrm{C}(6) & 120.8(9) \\ \mathrm{B}(1)-\mathrm{N}(1)-\mathrm{C}(6) & 130.6(9) \\ \mathrm{C}(5)-\mathrm{N}(1)-\mathrm{Fe}(1) & 68.0(5) \\ \mathrm{B}(1)-\mathrm{N}(1)-\mathrm{Fe}(1) & 76.9(6) \\ \mathrm{C}(6)-\mathrm{N}(1)-\mathrm{Fe}(1) & 130.1(6) \\ \mathrm{C}(27)-\mathrm{C}(26)-\mathrm{N}(2) & 108.4(8) \\ \mathrm{C}(27)-\mathrm{C}(26)-\mathrm{C}(29) & 112.4(10) \\ \mathrm{N}(2)-\mathrm{C}(26)-\mathrm{C}(29) & 106.5(8) \\ \mathrm{C}(27)-\mathrm{C}(26)-\mathrm{C}(28) & 109.8(9) \\ \mathrm{N}(2)-\mathrm{C}(26)-\mathrm{C}(28) & 110.4(8) \\ \mathrm{C}(29)-\mathrm{C}(26)-\mathrm{C}(28) & 109.3(10) \\ \mathrm{O}(3)-\mathrm{C}(11)-\mathrm{Fe}(1) & 178.2(10) \\ \mathrm{O}(2)-\mathrm{C}(10)-\mathrm{Fe}(1) & 179.2(10) \\ \mathrm{C}(25)-\mathrm{N}(2)-\mathrm{B}(2) & 107.7(8) \\ \mathrm{C}(25)-\mathrm{N}(2)-\mathrm{C}(26) & 123.1(8) \\ \mathrm{B}(2)-\mathrm{N}(2)-\mathrm{C}(26) & 128.0(8) \\ \mathrm{C}(25)-\mathrm{N}(2)-\mathrm{Fe}(2) & 66.6(5) \\ \mathrm{B}(2)-\mathrm{N}(2)-\mathrm{Fe}(2) & 76.6(5) \\ & \end{array}$




\begin{tabular}{|c|c|}
\hline $\mathrm{C}(26)-\mathrm{N}(2)-\mathrm{Fe}(2)$ & $131.8(6)$ \\
\hline $\mathrm{O}(5)-\mathrm{C}(30)-\mathrm{Fe}(2)$ & $178.6(10)$ \\
\hline$C(25)-C(24)-C(23)$ & 111.1(10) \\
\hline$C(25)-C(24)-F e(2)$ & $68.8(6)$ \\
\hline $\mathrm{C}(23)-\mathrm{C}(24)-\mathrm{Fe}(2)$ & $73.3(6)$ \\
\hline$C(24)-C(23)-B(2)$ & 106.2(9) \\
\hline$C(24)-C(23)-F e(2)$ & $67.6(6)$ \\
\hline $\mathrm{B}(2)-\mathrm{C}(23)-\mathrm{Fe}(2)$ & $75.1(6)$ \\
\hline $\mathrm{O}(4)-\mathrm{B}(2)-\mathrm{N}(2)$ & $122.2(10)$ \\
\hline $\mathrm{O}(4)-\mathrm{B}(2)-\mathrm{C}(23)$ & 133.1(11) \\
\hline $\mathrm{N}(2)-\mathrm{B}(2)-\mathrm{C}(23)$ & $104.7(9)$ \\
\hline $\mathrm{O}(4)-\mathrm{B}(2)-\mathrm{Fe}(2)$ & $133.9(8)$ \\
\hline $\mathrm{N}(2)-\mathrm{B}(2)-\mathrm{Fe}(2)$ & $64.5(5)$ \\
\hline$C(23)-B(2)-F e(2)$ & $65.0(6)$ \\
\hline $\mathrm{C}(24)-\mathrm{C}(25)-\mathrm{N}(2)$ & 109.6(9) \\
\hline $\mathrm{C}(24)-\mathrm{C}(25)-\mathrm{Fe}(2)$ & $71.8(6)$ \\
\hline $\mathrm{N}(2)-\mathrm{C}(25)-\mathrm{Fe}(2)$ & $73.3(5)$ \\
\hline$C(4)-C(5)-N(1)$ & $111.7(11)$ \\
\hline $\mathrm{C}(4)-\mathrm{C}(5)-\mathrm{Fe}(1)$ & $72.2(7)$ \\
\hline $\mathrm{N}(1)-\mathrm{C}(5)-\mathrm{Fe}(1)$ & $72.8(6)$ \\
\hline $\mathrm{C}(9)-\mathrm{C}(6)-\mathrm{N}(1)$ & 108.8(9) \\
\hline$C(9)-C(6)-C(7)$ & $111.5(13)$ \\
\hline $\mathrm{N}(1)-\mathrm{C}(6)-\mathrm{C}(7)$ & 109.7(9) \\
\hline$C(9)-C(6)-C(8)$ & 111.1(10) \\
\hline $\mathrm{N}(1)-\mathrm{C}(6)-\mathrm{C}(8)$ & 107.6(9) \\
\hline$C(7)-C(6)-C(8)$ & 108.1(11) \\
\hline$C(4)-C(3)-B(1)$ & $103.3(12)$ \\
\hline $\mathrm{C}(4)-\mathrm{C}(3)-\mathrm{Fe}(1)$ & $68.1(7)$ \\
\hline $\mathrm{B}(1)-\mathrm{C}(3)-\mathrm{Fe}(1)$ & $75.4(7)$ \\
\hline $\mathrm{O}(6)-\mathrm{C}(31)-\mathrm{Fe}(2)$ & $177.0(10)$ \\
\hline$C(5)-C(4)-C(3)$ & $110.0(12)$ \\
\hline$C(5)-C(4)-F e(1)$ & $70.3(7)$ \\
\hline $\mathrm{C}(3)-\mathrm{C}(4)-\mathrm{Fe}(1)$ & $71.3(7)$ \\
\hline $\mathrm{O}(1)-\mathrm{B}(1)-\mathrm{N}(1)$ & $123.2(11)$ \\
\hline $\mathrm{O}(1)-\mathrm{B}(1)-\mathrm{C}(3)$ & $130.2(13)$ \\
\hline $\mathrm{N}(1)-\mathrm{B}(1)-\mathrm{C}(3)$ & $106.5(12)$ \\
\hline $\mathrm{O}(1)-\mathrm{B}(1)-\mathrm{Fe}(1)$ & $130.8(10)$ \\
\hline $\mathrm{N}(1)-\mathrm{B}(1)-\mathrm{Fe}(1)$ & $65.4(6)$ \\
\hline $\mathrm{C}(3)-\mathrm{B}(1)-\mathrm{Fe}(1)$ & $64.8(6)$ \\
\hline $\mathrm{C}(53)-\mathrm{O}(7)-\mathrm{C}(50)$ & $105(2)$ \\
\hline$C(50)-C(51)-C(52)$ & $100(4)$ \\
\hline $\mathrm{O}(7)-\mathrm{C}(53)-\mathrm{C}(52)$ & 104(3) \\
\hline $\mathrm{C}(51)-\mathrm{C}(50)-\mathrm{O}(7)$ & $112(4)$ \\
\hline C(51)-C(52)-C(53) & 118(5) \\
\hline
\end{tabular}


Table 4. Anisotropic displacement parameters $\left(\AA^{2} \times 10^{3}\right)$ for 03199SL0. The anisotropic displacement factor exponent takes the form: $-2 p^{2}\left[h^{2} a^{* 2} U^{11}+\ldots+2 h k a^{*} b^{*} U^{12}\right]$

\begin{tabular}{|c|c|c|c|c|c|c|}
\hline & $\mathrm{U}^{11}$ & $\mathrm{U}^{22}$ & $\mathrm{U}^{33}$ & $\mathrm{U}^{23}$ & $\mathrm{U}^{13}$ & $\mathrm{U}^{12}$ \\
\hline $\mathrm{Fe}(1)$ & $36(1)$ & $32(1)$ & $41(1)$ & $4(1)$ & $2(1)$ & $-2(1)$ \\
\hline $\mathrm{Fe}(2)$ & $29(1)$ & $35(1)$ & $35(1)$ & $0(1)$ & $-2(1)$ & $-2(1)$ \\
\hline $\operatorname{Si}(1)$ & $47(2)$ & $30(2)$ & $47(2)$ & $-3(2)$ & $1(2)$ & $1(1)$ \\
\hline $\operatorname{Si}(2)$ & $38(2)$ & $40(2)$ & $39(2)$ & $1(2)$ & $-8(1)$ & $-1(1)$ \\
\hline $\mathrm{O}(3)$ & $69(6)$ & $67(6)$ & $54(5)$ & $8(4)$ & $17(5)$ & $-15(5)$ \\
\hline $\mathrm{N}(1)$ & $45(5)$ & $33(5)$ & $46(6)$ & $10(4)$ & $4(5)$ & $-12(4)$ \\
\hline $\mathrm{O}(5)$ & $36(4)$ & $83(6)$ & $64(5)$ & $-25(5)$ & $11(4)$ & $3(4)$ \\
\hline$C(26)$ & $38(6)$ & $47(7)$ & $41(7)$ & $3(6)$ & $2(5)$ & $-1(5)$ \\
\hline$C(11)$ & $45(6)$ & $26(6)$ & $45(7)$ & $3(6)$ & $-15(5)$ & $8(5)$ \\
\hline$C(10)$ & $54(7)$ & $44(7)$ & $49(7)$ & $0(6)$ & $-2(6)$ & $7(6)$ \\
\hline $\mathrm{N}(2)$ & $34(5)$ & $35(5)$ & $27(4)$ & $4(4)$ & $1(4)$ & $3(4)$ \\
\hline$C(30)$ & $41(6)$ & $50(7)$ & $18(5)$ & $-11(5)$ & $-14(5)$ & $-16(6)$ \\
\hline$C(24)$ & $32(6)$ & $56(7)$ & $50(7)$ & $9(6)$ & $-2(5)$ & $-15(6)$ \\
\hline $\mathrm{O}(2)$ & $87(6)$ & $69(5)$ & $61(5)$ & $-12(5)$ & $-20(5)$ & $13(5)$ \\
\hline$C(32)$ & $47(6)$ & $66(7)$ & $36(6)$ & $8(5)$ & $-7(6)$ & $-8(6)$ \\
\hline $\mathrm{O}(6)$ & $77(6)$ & $38(5)$ & $59(5)$ & $-4(4)$ & $-15(4)$ & $-19(4)$ \\
\hline$C(23)$ & $52(7)$ & $46(7)$ & $38(6)$ & $-6(6)$ & $6(6)$ & $-7(6)$ \\
\hline $\mathrm{B}(2)$ & $41(8)$ & $38(8)$ & $51(8)$ & $15(7)$ & $-5(7)$ & $0(7)$ \\
\hline$C(14)$ & $64(7)$ & $24(6)$ & $80(9)$ & $0(6)$ & $-7(7)$ & $-10(5)$ \\
\hline$C(25)$ & $35(6)$ & $41(6)$ & $38(6)$ & $3(5)$ & $-3(5)$ & $-7(5)$ \\
\hline$C(33)$ & $69(8)$ & $74(8)$ & $28(6)$ & $18(6)$ & $-10(6)$ & $-7(7)$ \\
\hline$C(5)$ & $61(9)$ & $41(7)$ & $70(8)$ & $18(7)$ & $-14(8)$ & $-17(7)$ \\
\hline$C(6)$ & $69(8)$ & $27(6)$ & $48(7)$ & $-6(5)$ & $15(6)$ & $7(6)$ \\
\hline$C(28)$ & $67(8)$ & $64(8)$ & $42(7)$ & $-24(6)$ & $-5(6)$ & $7(7)$ \\
\hline$C(8)$ & $105(12)$ & $48(8)$ & $97(10)$ & $4(8)$ & $2(9)$ & $-10(8)$ \\
\hline$C(13)$ & $74(9)$ & $34(6)$ & $95(9)$ & $-1(7)$ & $-13(8)$ & $3(7)$ \\
\hline$C(12)$ & $73(9)$ & $55(7)$ & $71(8)$ & $-12(7)$ & $8(7)$ & $9(7)$ \\
\hline$C(3)$ & $54(8)$ & $34(7)$ & $128(13)$ & $13(8)$ & $38(9)$ & $-7(6)$ \\
\hline$C(27)$ & $81(9)$ & $65(8)$ & $56(8)$ & $18(7)$ & $-21(7)$ & $-12(8)$ \\
\hline$C(31)$ & $33(6)$ & $68(8)$ & $42(7)$ & $1(6)$ & $-6(5)$ & $-8(6)$ \\
\hline$C(4)$ & $36(7)$ & $51(9)$ & $137(14)$ & $21(9)$ & $-29(8)$ & $-23(6)$ \\
\hline$C(34)$ & $51(7)$ & $46(7)$ & $64(8)$ & $-3(6)$ & $-10(6)$ & $22(6)$ \\
\hline$C(7)$ & $250(20)$ & $66(9)$ & $66(10)$ & $0(8)$ & $86(12)$ & $38(12)$ \\
\hline $\mathrm{B}(1)$ & $64(10)$ & $37(8)$ & $76(11)$ & $16(8)$ & $24(9)$ & $-16(7)$ \\
\hline$C(29)$ & $38(7)$ & $88(9)$ & $59(8)$ & $-16(7)$ & $-7(6)$ & $2(6)$ \\
\hline $\mathrm{O}(1)$ & 149(9) & $41(5)$ & $54(5)$ & $6(4)$ & $28(6)$ & $-5(5)$ \\
\hline$C(9)$ & $62(8)$ & $92(10)$ & $148(14)$ & $-42(11)$ & $-7(10)$ & $24(8)$ \\
\hline $\mathrm{O}(4)$ & $73(6)$ & $37(4)$ & $51(5)$ & $-12(4)$ & $-3(4)$ & $14(4)$ \\
\hline $\mathrm{O}(7)$ & $510(50)$ & 181(17) & 162(17) & $-24(14)$ & $-90(20)$ & $-10(20)$ \\
\hline$C(51)$ & $280(50)$ & $560(100)$ & $690(90)$ & $70(80)$ & $210(60)$ & $-180(60)$ \\
\hline$C(53)$ & $640(100)$ & $270(40)$ & 101(19) & $-60(20)$ & $-70(40)$ & $0(50)$ \\
\hline$C(50)$ & $790(130)$ & $26(14)$ & $640(80)$ & $-30(30)$ & $-240(80)$ & $50(40)$ \\
\hline
\end{tabular}


$\begin{array}{llllll}\mathrm{C}(52) & 290(50) & 420(60) & 410(70) & 340(60) & -10(50)\end{array}$ 
Table 5. Hydrogen coordinates $\left(x 10^{4}\right)$ and isotropic displacement parameters $\left(\AA^{2} \times 10^{3}\right)$ for 03199SL0.

\begin{tabular}{|c|c|c|c|c|}
\hline & $x$ & $\mathrm{y}$ & $\mathrm{z}$ & $\mathrm{U}(\mathrm{eq})$ \\
\hline $\mathrm{H}(24)$ & 7792 & 4078 & 5924 & 55 \\
\hline $\mathrm{H}(32 \mathrm{~A})$ & 6666 & 3733 & 7158 & 75 \\
\hline $\mathrm{H}(32 \mathrm{~B})$ & 4949 & 4310 & 6994 & 75 \\
\hline $\mathrm{H}(32 \mathrm{C})$ & 7066 & 4313 & 6771 & 75 \\
\hline $\mathrm{H}(23)$ & 5504 & 5188 & 6191 & 55 \\
\hline $\mathrm{H}(14 \mathrm{~A})$ & 9518 & 6020 & 8386 & 84 \\
\hline $\mathrm{H}(14 \mathrm{~B})$ & 10746 & 5347 & 8145 & 84 \\
\hline $\mathrm{H}(14 \mathrm{C})$ & 10594 & 5328 & 8643 & 84 \\
\hline $\mathrm{H}(25)$ & 6322 & 3424 & 5287 & 45 \\
\hline $\mathrm{H}(33 \mathrm{~A})$ & 2639 & 2220 & 6669 & 85 \\
\hline $\mathrm{H}(33 \mathrm{~B})$ & 2422 & 2947 & 6989 & 85 \\
\hline $\mathrm{H}(33 \mathrm{C})$ & 4056 & 2274 & 7068 & 85 \\
\hline $\mathrm{H}(5)$ & 6497 & 2686 & 7656 & 68 \\
\hline $\mathrm{H}(28 \mathrm{~A})$ & 4683 & 3263 & 4669 & 86 \\
\hline $\mathrm{H}(28 \mathrm{~B})$ & 2425 & 3100 & 4545 & 86 \\
\hline $\mathrm{H}(28 \mathrm{C})$ & 3221 & 2853 & 4997 & 86 \\
\hline $\mathrm{H}(8 \mathrm{~A})$ & 8451 & 348 & 7951 & 126 \\
\hline $\mathrm{H}(8 \mathrm{~B})$ & 6502 & 863 & 7863 & 126 \\
\hline $\mathrm{H}(8 \mathrm{C})$ & 7168 & 684 & 8333 & 126 \\
\hline $\mathrm{H}(13 \mathrm{~A})$ & 5114 & 5554 & 7976 & 102 \\
\hline $\mathrm{H}(13 \mathrm{~B})$ & 5654 & 4761 & 7723 & 102 \\
\hline $\mathrm{H}(13 \mathrm{C})$ & 7099 & 5516 & 7705 & 102 \\
\hline $\mathrm{H}(12 \mathrm{~A})$ & 5919 & 5667 & 8815 & 100 \\
\hline $\mathrm{H}(12 \mathrm{~B})$ & 6598 & 4871 & 9050 & 100 \\
\hline $\mathrm{H}(12 \mathrm{C})$ & 4689 & 4860 & 8754 & 100 \\
\hline $\mathrm{H}(3)$ & 4971 & 3364 & 8869 & 86 \\
\hline $\mathrm{H}(27 \mathrm{~A})$ & 2807 & 5216 & 4726 & 101 \\
\hline $\mathrm{H}(27 \mathrm{~B})$ & 2594 & 4573 & 4360 & 101 \\
\hline $\mathrm{H}(27 \mathrm{C})$ & 4689 & 4707 & 4582 & 101 \\
\hline $\mathrm{H}(4)$ & 4338 & 3582 & 8061 & 90 \\
\hline $\mathrm{H}(34 \mathrm{~A})$ & 7991 & 2343 & 6687 & 80 \\
\hline $\mathrm{H}(34 \mathrm{~B})$ & 8241 & 2734 & 6235 & 80 \\
\hline $\mathrm{H}(34 \mathrm{C})$ & 6794 & 1989 & 6298 & 80 \\
\hline $\mathrm{H}(7 \mathrm{~A})$ & 10495 & 2167 & 7605 & 190 \\
\hline $\mathrm{H}(7 \mathrm{~B})$ & 8276 & 1976 & 7462 & 190 \\
\hline $\mathrm{H}(7 \mathrm{C})$ & 9901 & 1283 & 7467 & 190 \\
\hline $\mathrm{H}(29 \mathrm{~A})$ & 285 & 3625 & 5245 & 93 \\
\hline $\mathrm{H}(29 B)$ & -234 & 4009 & 4801 & 93 \\
\hline $\mathrm{H}(29 \mathrm{C})$ & 225 & 4572 & 5192 & 93 \\
\hline $\mathrm{H}(1 \mathrm{~A})$ & 7180 & 2116 & 9212 & 98 \\
\hline $\mathrm{H}(9 \mathrm{~A})$ & 11458 & 1026 & 8216 & 151 \\
\hline
\end{tabular}




$\begin{array}{lllll}\mathrm{H}(9 \mathrm{~B}) & 10280 & 1391 & 8604 & 151 \\ \mathrm{H}(9 \mathrm{C}) & 11484 & 1966 & 8298 & 151 \\ \mathrm{H}(4 \mathrm{~A}) & 2184 & 5818 & 5765 & 64 \\ \mathrm{H}(51 \mathrm{~A}) & 8543 & 5832 & 5395 & 613 \\ \mathrm{H}(51 \mathrm{~B}) & 6210 & 5895 & 5290 & 613 \\ \mathrm{H}(53 \mathrm{~A}) & 6553 & 7993 & 5153 & 404 \\ \mathrm{H}(53 \mathrm{~B}) & 8891 & 7979 & 5252 & 404 \\ \mathrm{H}(50 \mathrm{~A}) & 6955 & 6149 & 4641 & 580 \\ \mathrm{H}(50 \mathrm{~B}) & 9264 & 6125 & 4751 & 580 \\ \mathrm{H}(52 \mathrm{~A}) & 8481 & 6962 & 5666 & 446 \\ \mathrm{H}(52 \mathrm{~B}) & 6156 & 7004 & 5587 & 446 \\ -\end{array}$


Table 6. Torsion angles [ $\left.{ }^{\circ}\right]$ for 03199SL0.

\begin{tabular}{|c|c|}
\hline $\mathrm{C}(10)-\mathrm{Fe}(1)-\mathrm{Si}(1)-\mathrm{C}(12)$ & $-76.3(6)$ \\
\hline$C(11)-F e(1)-S i(1)-C(12)$ & $-172.5(6)$ \\
\hline$C(5)-\mathrm{Fe}(1)-\mathrm{Si}(1)-\mathrm{C}(12)$ & $92.6(6)$ \\
\hline$C(4)-F e(1)-S i(1)-C(12)$ & $67.3(7)$ \\
\hline$N(1)-F e(1)-S i(1)-C(12)$ & $68.3(8)$ \\
\hline $\mathrm{C}(3)-\mathrm{Fe}(1)-\mathrm{Si}(1)-\mathrm{C}(12)$ & $26.8(7)$ \\
\hline $\mathrm{B}(1)-\mathrm{Fe}(1)-\mathrm{Si}(1)-\mathrm{C}(12)$ & $12.1(7)$ \\
\hline$C(10)-F e(1)-S i(1)-C(13)$ & $165.0(6)$ \\
\hline$C(11)-F e(1)-S i(1)-C(13)$ & $68.8(6)$ \\
\hline $\mathrm{C}(5)-\mathrm{Fe}(1)-\mathrm{Si}(1)-\mathrm{C}(13)$ & $-26.1(6)$ \\
\hline$C(4)-F e(1)-S i(1)-C(13)$ & $-51.4(7)$ \\
\hline $\mathrm{N}(1)-\mathrm{Fe}(1)-\mathrm{Si}(1)-\mathrm{C}(13)$ & $-50.3(8)$ \\
\hline$C(3)-F e(1)-S i(1)-C(13)$ & $-91.8(7)$ \\
\hline $\mathrm{B}(1)-\mathrm{Fe}(1)-\mathrm{Si}(1)-\mathrm{C}(13)$ & $-106.5(7)$ \\
\hline$C(10)-F e(1)-S i(1)-C(14)$ & $44.9(5)$ \\
\hline$C(11)-F e(1)-S i(1)-C(14)$ & $-51.3(5)$ \\
\hline $\mathrm{C}(5)-\mathrm{Fe}(1)-\mathrm{Si}(1)-\mathrm{C}(14)$ & $-146.2(6)$ \\
\hline$C(4)-F e(1)-S i(1)-C(14)$ & $-171.5(6)$ \\
\hline $\mathrm{N}(1)-\mathrm{Fe}(1)-\mathrm{Si}(1)-\mathrm{C}(14)$ & $-170.5(7)$ \\
\hline $\mathrm{C}(3)-\mathrm{Fe}(1)-\mathrm{Si}(1)-\mathrm{C}(14)$ & $148.0(6)$ \\
\hline $\mathrm{B}(1)-\mathrm{Fe}(1)-\mathrm{Si}(1)-\mathrm{C}(14)$ & 133.3(7) \\
\hline$C(31)-F e(2)-S i(2)-C(34)$ & $63.6(5)$ \\
\hline$C(30)-F e(2)-S i(2)-C(34)$ & $164.4(5)$ \\
\hline$C(25)-F e(2)-S i(2)-C(34)$ & $-31.1(5)$ \\
\hline$C(24)-F e(2)-S i(2)-C(34)$ & $-61.5(5)$ \\
\hline $\mathrm{N}(2)-\mathrm{Fe}(2)-\mathrm{Si}(2)-\mathrm{C}(34)$ & $-45.5(7)$ \\
\hline$C(23)-F e(2)-S i(2)-C(34)$ & $-99.6(5)$ \\
\hline $\mathrm{B}(2)-\mathrm{Fe}(2)-\mathrm{Si}(2)-\mathrm{C}(34)$ & $-112.4(6)$ \\
\hline$C(31)-F e(2)-S i(2)-C(33)$ & $-55.9(5)$ \\
\hline$C(30)-F e(2)-S i(2)-C(33)$ & $44.8(5)$ \\
\hline$C(25)-F e(2)-S i(2)-C(33)$ & $-150.6(5)$ \\
\hline$C(24)-F e(2)-S i(2)-C(33)$ & $179.0(5)$ \\
\hline $\mathrm{N}(2)-\mathrm{Fe}(2)-\mathrm{Si}(2)-\mathrm{C}(33)$ & $-165.1(6)$ \\
\hline$C(23)-F e(2)-S i(2)-C(33)$ & $140.9(5)$ \\
\hline $\mathrm{B}(2)-\mathrm{Fe}(2)-\mathrm{Si}(2)-\mathrm{C}(33)$ & $128.1(6)$ \\
\hline$C(31)-F e(2)-S i(2)-C(32)$ & $-172.9(5)$ \\
\hline$C(30)-F e(2)-S i(2)-C(32)$ & $-72.1(5)$ \\
\hline$C(25)-F e(2)-S i(2)-C(32)$ & $92.4(5)$ \\
\hline$C(24)-F e(2)-S i(2)-C(32)$ & $62.0(5)$ \\
\hline $\mathrm{N}(2)-\mathrm{Fe}(2)-\mathrm{Si}(2)-\mathrm{C}(32)$ & $78.0(7)$ \\
\hline$C(23)-F e(2)-S i(2)-C(32)$ & $23.9(5)$ \\
\hline $\mathrm{B}(2)-\mathrm{Fe}(2)-\mathrm{Si}(2)-\mathrm{C}(32)$ & $11.1(6)$ \\
\hline $\mathrm{C}(10)-\mathrm{Fe}(1)-\mathrm{N}(1)-\mathrm{C}(5)$ & $175.4(7)$ \\
\hline$C(11)-F e(1)-N(1)-C(5)$ & $-80.2(8)$ \\
\hline $\mathrm{C}(4)-\mathrm{Fe}(1)-\mathrm{N}(1)-\mathrm{C}(5)$ & $35.3(7)$ \\
\hline
\end{tabular}




\begin{tabular}{|c|c|}
\hline $\mathrm{C}(3)-\mathrm{Fe}(1)-\mathrm{N}(1)-\mathrm{C}(5)$ & $79.9(8)$ \\
\hline $\mathrm{B}(1)-\mathrm{Fe}(1)-\mathrm{N}(1)-\mathrm{C}(5)$ & $115.0(10)$ \\
\hline $\mathrm{Si}(1)-\mathrm{Fe}(1)-\mathrm{N}(1)-\mathrm{C}(5)$ & $34.2(10)$ \\
\hline $\mathrm{C}(10)-\mathrm{Fe}(1)-\mathrm{N}(1)-\mathrm{B}(1)$ & $60.4(8)$ \\
\hline $\mathrm{C}(11)-\mathrm{Fe}(1)-\mathrm{N}(1)-\mathrm{B}(1)$ & $164.8(8)$ \\
\hline $\mathrm{C}(5)-\mathrm{Fe}(1)-\mathrm{N}(1)-\mathrm{B}(1)$ & $-115.0(10)$ \\
\hline $\mathrm{C}(4)-\mathrm{Fe}(1)-\mathrm{N}(1)-\mathrm{B}(1)$ & $-79.7(9)$ \\
\hline $\mathrm{C}(3)-\mathrm{Fe}(1)-\mathrm{N}(1)-\mathrm{B}(1)$ & $-35.2(8)$ \\
\hline $\mathrm{Si}(1)-\mathrm{Fe}(1)-\mathrm{N}(1)-\mathrm{B}(1)$ & $-80.8(9)$ \\
\hline $\mathrm{C}(10)-\mathrm{Fe}(1)-\mathrm{N}(1)-\mathrm{C}(6)$ & $-72.2(9)$ \\
\hline $\mathrm{C}(11)-\mathrm{Fe}(1)-\mathrm{N}(1)-\mathrm{C}(6)$ & $32.2(9)$ \\
\hline $\mathrm{C}(5)-\mathrm{Fe}(1)-\mathrm{N}(1)-\mathrm{C}(6)$ & $112.4(11)$ \\
\hline $\mathrm{C}(4)-\mathrm{Fe}(1)-\mathrm{N}(1)-\mathrm{C}(6)$ & $147.7(10)$ \\
\hline$C(3)-F e(1)-N(1)-C(6)$ & $-167.7(10)$ \\
\hline $\mathrm{B}(1)-\mathrm{Fe}(1)-\mathrm{N}(1)-\mathrm{C}(6)$ & $-132.6(12)$ \\
\hline $\mathrm{Si}(1)-\mathrm{Fe}(1)-\mathrm{N}(1)-\mathrm{C}(6)$ & $146.6(7)$ \\
\hline $\mathrm{C}(10)-\mathrm{Fe}(1)-\mathrm{C}(11)-\mathrm{O}(3)$ & $-75(28)$ \\
\hline $\mathrm{C}(5)-\mathrm{Fe}(1)-\mathrm{C}(11)-\mathrm{O}(3)$ & $130(28)$ \\
\hline $\mathrm{C}(4)-\mathrm{Fe}(1)-\mathrm{C}(11)-\mathrm{O}(3)$ & $98(28)$ \\
\hline $\mathrm{N}(1)-\mathrm{Fe}(1)-\mathrm{C}(11)-\mathrm{O}(3)$ & $169(100)$ \\
\hline $\mathrm{C}(3)-\mathrm{Fe}(1)-\mathrm{C}(11)-\mathrm{O}(3)$ & $96(28)$ \\
\hline $\mathrm{B}(1)-\mathrm{Fe}(1)-\mathrm{C}(11)-\mathrm{O}(3)$ & $-176(100)$ \\
\hline $\mathrm{Si}(1)-\mathrm{Fe}(1)-\mathrm{C}(11)-\mathrm{O}(3)$ & $10(28)$ \\
\hline $\mathrm{C}(11)-\mathrm{Fe}(1)-\mathrm{C}(10)-\mathrm{O}(2)$ & $147(84)$ \\
\hline $\mathrm{C}(5)-\mathrm{Fe}(1)-\mathrm{C}(10)-\mathrm{O}(2)$ & $-95(84)$ \\
\hline $\mathrm{C}(4)-\mathrm{Fe}(1)-\mathrm{C}(10)-\mathrm{O}(2)$ & $-22(84)$ \\
\hline $\mathrm{N}(1)-\mathrm{Fe}(1)-\mathrm{C}(10)-\mathrm{O}(2)$ & $-101(84)$ \\
\hline $\mathrm{C}(3)-\mathrm{Fe}(1)-\mathrm{C}(10)-\mathrm{O}(2)$ & $-30(84)$ \\
\hline $\mathrm{B}(1)-\mathrm{Fe}(1)-\mathrm{C}(10)-\mathrm{O}(2)$ & $-69(84)$ \\
\hline $\mathrm{Si}(1)-\mathrm{Fe}(1)-\mathrm{C}(10)-\mathrm{O}(2)$ & $64(84)$ \\
\hline $\mathrm{C}(27)-\mathrm{C}(26)-\mathrm{N}(2)-\mathrm{C}(25)$ & $-98.5(11)$ \\
\hline $\mathrm{C}(29)-\mathrm{C}(26)-\mathrm{N}(2)-\mathrm{C}(25)$ & $140.3(9)$ \\
\hline $\mathrm{C}(28)-\mathrm{C}(26)-\mathrm{N}(2)-\mathrm{C}(25)$ & $21.8(12)$ \\
\hline $\mathrm{C}(27)-\mathrm{C}(26)-\mathrm{N}(2)-\mathrm{B}(2)$ & $67.0(13)$ \\
\hline$C(29)-C(26)-N(2)-B(2)$ & $-54.2(13)$ \\
\hline $\mathrm{C}(28)-\mathrm{C}(26)-\mathrm{N}(2)-\mathrm{B}(2)$ & $-172.7(9)$ \\
\hline $\mathrm{C}(27)-\mathrm{C}(26)-\mathrm{N}(2)-\mathrm{Fe}(2)$ & $174.7(7)$ \\
\hline $\mathrm{C}(29)-\mathrm{C}(26)-\mathrm{N}(2)-\mathrm{Fe}(2)$ & $53.5(12)$ \\
\hline $\mathrm{C}(28)-\mathrm{C}(26)-\mathrm{N}(2)-\mathrm{Fe}(2)$ & $-65.1(11)$ \\
\hline $\mathrm{C}(31)-\mathrm{Fe}(2)-\mathrm{N}(2)-\mathrm{C}(25)$ & $-83.5(6)$ \\
\hline $\mathrm{C}(30)-\mathrm{Fe}(2)-\mathrm{N}(2)-\mathrm{C}(25)$ & $166.7(6)$ \\
\hline $\mathrm{C}(24)-\mathrm{Fe}(2)-\mathrm{N}(2)-\mathrm{C}(25)$ & $37.8(6)$ \\
\hline $\mathrm{C}(23)-\mathrm{Fe}(2)-\mathrm{N}(2)-\mathrm{C}(25)$ & $80.4(6)$ \\
\hline $\mathrm{B}(2)-\mathrm{Fe}(2)-\mathrm{N}(2)-\mathrm{C}(25)$ & $116.3(8)$ \\
\hline $\mathrm{Si}(2)-\mathrm{Fe}(2)-\mathrm{N}(2)-\mathrm{C}(25)$ & $20.4(8)$ \\
\hline $\mathrm{C}(31)-\mathrm{Fe}(2)-\mathrm{N}(2)-\mathrm{B}(2)$ & $160.2(7)$ \\
\hline $\mathrm{C}(30)-\mathrm{Fe}(2)-\mathrm{N}(2)-\mathrm{B}(2)$ & $50.4(7)$ \\
\hline
\end{tabular}




\begin{tabular}{|c|c|}
\hline$C(25)-\mathrm{Fe}(2)-\mathrm{N}(2)-\mathrm{B}(2)$ & $-116.3(8)$ \\
\hline $\mathrm{C}(24)-\mathrm{Fe}(2)-\mathrm{N}(2)-\mathrm{B}(2)$ & $-78.5(7)$ \\
\hline $\mathrm{C}(23)-\mathrm{Fe}(2)-\mathrm{N}(2)-\mathrm{B}(2)$ & $-36.0(6)$ \\
\hline $\mathrm{Si}(2)-\mathrm{Fe}(2)-\mathrm{N}(2)-\mathrm{B}(2)$ & $-96.0(7)$ \\
\hline $\mathrm{C}(31)-\mathrm{Fe}(2)-\mathrm{N}(2)-\mathrm{C}(26)$ & $30.7(9)$ \\
\hline $\mathrm{C}(30)-\mathrm{Fe}(2)-\mathrm{N}(2)-\mathrm{C}(26)$ & $-79.0(9)$ \\
\hline $\mathrm{C}(25)-\mathrm{Fe}(2)-\mathrm{N}(2)-\mathrm{C}(26)$ & $114.2(10)$ \\
\hline $\mathrm{C}(24)-\mathrm{Fe}(2)-\mathrm{N}(2)-\mathrm{C}(26)$ & $152.0(9)$ \\
\hline $\mathrm{C}(23)-\mathrm{Fe}(2)-\mathrm{N}(2)-\mathrm{C}(26)$ & $-165.4(9)$ \\
\hline $\mathrm{B}(2)-\mathrm{Fe}(2)-\mathrm{N}(2)-\mathrm{C}(26)$ & $-129.4(11)$ \\
\hline $\mathrm{Si}(2)-\mathrm{Fe}(2)-\mathrm{N}(2)-\mathrm{C}(26)$ & $134.6(7)$ \\
\hline $\mathrm{C}(31)-\mathrm{Fe}(2)-\mathrm{C}(30)-\mathrm{O}(5)$ & $-127(38)$ \\
\hline $\mathrm{C}(25)-\mathrm{Fe}(2)-\mathrm{C}(30)-\mathrm{O}(5)$ & $6(39)$ \\
\hline $\mathrm{C}(24)-\mathrm{Fe}(2)-\mathrm{C}(30)-\mathrm{O}(5)$ & $68(38)$ \\
\hline $\mathrm{N}(2)-\mathrm{Fe}(2)-\mathrm{C}(30)-\mathrm{O}(5)$ & $-14(38)$ \\
\hline $\mathrm{C}(23)-\mathrm{Fe}(2)-\mathrm{C}(30)-\mathrm{O}(5)$ & $55(38)$ \\
\hline $\mathrm{B}(2)-\mathrm{Fe}(2)-\mathrm{C}(30)-\mathrm{O}(5)$ & $15(38)$ \\
\hline $\mathrm{Si}(2)-\mathrm{Fe}(2)-\mathrm{C}(30)-\mathrm{O}(5)$ & $153(38)$ \\
\hline $\mathrm{C}(31)-\mathrm{Fe}(2)-\mathrm{C}(24)-\mathrm{C}(25)$ & $55.2(8)$ \\
\hline$C(30)-F e(2)-C(24)-C(25)$ & $-143.1(7)$ \\
\hline $\mathrm{N}(2)-\mathrm{Fe}(2)-\mathrm{C}(24)-\mathrm{C}(25)$ & $-38.4(6)$ \\
\hline$C(23)-F e(2)-C(24)-C(25)$ & $-121.3(9)$ \\
\hline $\mathrm{B}(2)-\mathrm{Fe}(2)-\mathrm{C}(24)-\mathrm{C}(25)$ & $-81.3(7)$ \\
\hline $\operatorname{Si}(2)-F e(2)-C(24)-C(25)$ & $134.3(6)$ \\
\hline$C(31)-F e(2)-C(24)-C(23)$ & $176.5(6)$ \\
\hline$C(30)-F e(2)-C(24)-C(23)$ & $-21.8(9)$ \\
\hline$C(25)-F e(2)-C(24)-C(23)$ & $121.3(9)$ \\
\hline $\mathrm{N}(2)-\mathrm{Fe}(2)-\mathrm{C}(24)-\mathrm{C}(23)$ & $82.9(6)$ \\
\hline $\mathrm{B}(2)-\mathrm{Fe}(2)-\mathrm{C}(24)-\mathrm{C}(23)$ & $40.0(6)$ \\
\hline $\operatorname{Si}(2)-F e(2)-C(24)-C(23)$ & $-104.4(6)$ \\
\hline $\mathrm{C}(25)-\mathrm{C}(24)-\mathrm{C}(23)-\mathrm{B}(2)$ & $-7.2(12)$ \\
\hline $\mathrm{Fe}(2)-\mathrm{C}(24)-\mathrm{C}(23)-\mathrm{B}(2)$ & $-65.8(7)$ \\
\hline$C(25)-C(24)-C(23)-F e(2)$ & $58.6(7)$ \\
\hline$C(31)-F e(2)-C(23)-C(24)$ & $-10.2(18)$ \\
\hline$C(30)-F e(2)-C(23)-C(24)$ & $164.4(6)$ \\
\hline$C(25)-\mathrm{Fe}(2)-\mathrm{C}(23)-\mathrm{C}(24)$ & $-36.2(6)$ \\
\hline $\mathrm{N}(2)-\mathrm{Fe}(2)-\mathrm{C}(23)-\mathrm{C}(24)$ & $-79.9(6)$ \\
\hline $\mathrm{B}(2)-\mathrm{Fe}(2)-\mathrm{C}(23)-\mathrm{C}(24)$ & $-115.0(9)$ \\
\hline $\operatorname{Si}(2)-F e(2)-C(23)-C(24)$ & $78.6(6)$ \\
\hline $\mathrm{C}(31)-\mathrm{Fe}(2)-\mathrm{C}(23)-\mathrm{B}(2)$ & $104.8(16)$ \\
\hline$C(30)-F e(2)-C(23)-B(2)$ & $-80.6(7)$ \\
\hline $\mathrm{C}(25)-\mathrm{Fe}(2)-\mathrm{C}(23)-\mathrm{B}(2)$ & $78.8(7)$ \\
\hline$C(24)-F e(2)-C(23)-B(2)$ & $115.0(9)$ \\
\hline $\mathrm{N}(2)-\mathrm{Fe}(2)-\mathrm{C}(23)-\mathrm{B}(2)$ & $35.1(6)$ \\
\hline $\operatorname{Si}(2)-F e(2)-C(23)-B(2)$ & $-166.5(6)$ \\
\hline $\mathrm{C}(25)-\mathrm{N}(2)-\mathrm{B}(2)-\mathrm{O}(4)$ & $172.9(9)$ \\
\hline $\mathrm{C}(26)-\mathrm{N}(2)-\mathrm{B}(2)-\mathrm{O}(4)$ & $5.6(16)$ \\
\hline
\end{tabular}




\begin{tabular}{|c|c|}
\hline $\mathrm{Fe}(2)-\mathrm{N}(2)-\mathrm{B}(2)-\mathrm{O}(4)$ & $-127.4(10)$ \\
\hline $\mathrm{C}(25)-\mathrm{N}(2)-\mathrm{B}(2)-\mathrm{C}(23)$ & $-7.2(10)$ \\
\hline $\mathrm{C}(26)-\mathrm{N}(2)-\mathrm{B}(2)-\mathrm{C}(23)$ & $-174.5(8)$ \\
\hline $\mathrm{Fe}(2)-\mathrm{N}(2)-\mathrm{B}(2)-\mathrm{C}(23)$ & $52.4(7)$ \\
\hline $\mathrm{C}(25)-\mathrm{N}(2)-\mathrm{B}(2)-\mathrm{Fe}(2)$ & $-59.7(6)$ \\
\hline $\mathrm{C}(26)-\mathrm{N}(2)-\mathrm{B}(2)-\mathrm{Fe}(2)$ & $133.1(9)$ \\
\hline $\mathrm{C}(24)-\mathrm{C}(23)-\mathrm{B}(2)-\mathrm{O}(4)$ & $-171.4(12)$ \\
\hline $\mathrm{Fe}(2)-\mathrm{C}(23)-\mathrm{B}(2)-\mathrm{O}(4)$ & $127.7(13)$ \\
\hline $\mathrm{C}(24)-\mathrm{C}(23)-\mathrm{B}(2)-\mathrm{N}(2)$ & $8.7(11)$ \\
\hline $\mathrm{Fe}(2)-\mathrm{C}(23)-\mathrm{B}(2)-\mathrm{N}(2)$ & $-52.1(7)$ \\
\hline$C(24)-C(23)-B(2)-F e(2)$ & $60.8(7)$ \\
\hline $\mathrm{C}(31)-\mathrm{Fe}(2)-\mathrm{B}(2)-\mathrm{O}(4)$ & $79.5(14)$ \\
\hline $\mathrm{C}(30)-\mathrm{Fe}(2)-\mathrm{B}(2)-\mathrm{O}(4)$ & $-25.2(12)$ \\
\hline $\mathrm{C}(25)-\mathrm{Fe}(2)-\mathrm{B}(2)-\mathrm{O}(4)$ & $150.5(13)$ \\
\hline $\mathrm{C}(24)-\mathrm{Fe}(2)-\mathrm{B}(2)-\mathrm{O}(4)$ & $-165.8(14)$ \\
\hline $\mathrm{N}(2)-\mathrm{Fe}(2)-\mathrm{B}(2)-\mathrm{O}(4)$ & $111.1(14)$ \\
\hline $\mathrm{C}(23)-\mathrm{Fe}(2)-\mathrm{B}(2)-\mathrm{O}(4)$ & $-126.7(15)$ \\
\hline $\mathrm{Si}(2)-\mathrm{Fe}(2)-\mathrm{B}(2)-\mathrm{O}(4)$ & $-106.8(11)$ \\
\hline $\mathrm{C}(31)-\mathrm{Fe}(2)-\mathrm{B}(2)-\mathrm{N}(2)$ & $-31.6(10)$ \\
\hline $\mathrm{C}(30)-\mathrm{Fe}(2)-\mathrm{B}(2)-\mathrm{N}(2)$ & $-136.3(6)$ \\
\hline $\mathrm{C}(25)-\mathrm{Fe}(2)-\mathrm{B}(2)-\mathrm{N}(2)$ & $39.4(5)$ \\
\hline $\mathrm{C}(24)-\mathrm{Fe}(2)-\mathrm{B}(2)-\mathrm{N}(2)$ & $83.1(6)$ \\
\hline $\mathrm{C}(23)-\mathrm{Fe}(2)-\mathrm{B}(2)-\mathrm{N}(2)$ & $122.2(9)$ \\
\hline $\mathrm{Si}(2)-\mathrm{Fe}(2)-\mathrm{B}(2)-\mathrm{N}(2)$ & $142.1(4)$ \\
\hline $\mathrm{C}(31)-\mathrm{Fe}(2)-\mathrm{B}(2)-\mathrm{C}(23)$ & $-153.8(7)$ \\
\hline$C(30)-F e(2)-B(2)-C(23)$ & $101.5(7)$ \\
\hline $\mathrm{C}(25)-\mathrm{Fe}(2)-\mathrm{B}(2)-\mathrm{C}(23)$ & $-82.8(7)$ \\
\hline $\mathrm{C}(24)-\mathrm{Fe}(2)-\mathrm{B}(2)-\mathrm{C}(23)$ & $-39.1(6)$ \\
\hline $\mathrm{N}(2)-\mathrm{Fe}(2)-\mathrm{B}(2)-\mathrm{C}(23)$ & $-122.2(9)$ \\
\hline $\operatorname{Si}(2)-F e(2)-B(2)-C(23)$ & $19.9(9)$ \\
\hline $\mathrm{C}(23)-\mathrm{C}(24)-\mathrm{C}(25)-\mathrm{N}(2)$ & $2.7(11)$ \\
\hline $\mathrm{Fe}(2)-\mathrm{C}(24)-\mathrm{C}(25)-\mathrm{N}(2)$ & $64.0(6)$ \\
\hline$C(23)-C(24)-C(25)-F e(2)$ & $-61.3(7)$ \\
\hline $\mathrm{B}(2)-\mathrm{N}(2)-\mathrm{C}(25)-\mathrm{C}(24)$ & $3.2(10)$ \\
\hline $\mathrm{C}(26)-\mathrm{N}(2)-\mathrm{C}(25)-\mathrm{C}(24)$ & $171.2(8)$ \\
\hline $\mathrm{Fe}(2)-\mathrm{N}(2)-\mathrm{C}(25)-\mathrm{C}(24)$ & $-63.0(7)$ \\
\hline $\mathrm{B}(2)-\mathrm{N}(2)-\mathrm{C}(25)-\mathrm{Fe}(2)$ & $66.2(6)$ \\
\hline $\mathrm{C}(26)-\mathrm{N}(2)-\mathrm{C}(25)-\mathrm{Fe}(2)$ & $-125.8(8)$ \\
\hline$C(31)-F e(2)-C(25)-C(24)$ & $-136.9(7)$ \\
\hline$C(30)-F e(2)-C(25)-C(24)$ & $89.5(10)$ \\
\hline $\mathrm{N}(2)-\mathrm{Fe}(2)-\mathrm{C}(25)-\mathrm{C}(24)$ & $117.8(8)$ \\
\hline$C(23)-F e(2)-C(25)-C(24)$ & $35.8(6)$ \\
\hline $\mathrm{B}(2)-\mathrm{Fe}(2)-\mathrm{C}(25)-\mathrm{C}(24)$ & $79.6(7)$ \\
\hline $\mathrm{Si}(2)-\mathrm{Fe}(2)-\mathrm{C}(25)-\mathrm{C}(24)$ & $-52.8(7)$ \\
\hline $\mathrm{C}(31)-\mathrm{Fe}(2)-\mathrm{C}(25)-\mathrm{N}(2)$ & $105.2(6)$ \\
\hline $\mathrm{C}(30)-\mathrm{Fe}(2)-\mathrm{C}(25)-\mathrm{N}(2)$ & $-28.4(11)$ \\
\hline $\mathrm{C}(24)-\mathrm{Fe}(2)-\mathrm{C}(25)-\mathrm{N}(2)$ & $-117.8(8)$ \\
\hline
\end{tabular}




\begin{tabular}{|c|c|}
\hline $\mathrm{C}(23)-\mathrm{Fe}(2)-\mathrm{C}(25)-\mathrm{N}(2)$ & $-82.0(5)$ \\
\hline $\mathrm{B}(2)-\mathrm{Fe}(2)-\mathrm{C}(25)-\mathrm{N}(2)$ & $-38.2(5)$ \\
\hline $\mathrm{Si}(2)-\mathrm{Fe}(2)-\mathrm{C}(25)-\mathrm{N}(2)$ & $-170.6(4)$ \\
\hline $\mathrm{B}(1)-\mathrm{N}(1)-\mathrm{C}(5)-\mathrm{C}(4)$ & $5.4(12)$ \\
\hline$C(6)-N(1)-C(5)-C(4)$ & 173.2(9) \\
\hline $\mathrm{Fe}(1)-\mathrm{N}(1)-\mathrm{C}(5)-\mathrm{C}(4)$ & $-62.1(8)$ \\
\hline $\mathrm{B}(1)-\mathrm{N}(1)-\mathrm{C}(5)-\mathrm{Fe}(1)$ & $67.5(7)$ \\
\hline$C(6)-N(1)-C(5)-F e(1)$ & $-124.7(8)$ \\
\hline$C(10)-\mathrm{Fe}(1)-\mathrm{C}(5)-\mathrm{C}(4)$ & $111.3(13)$ \\
\hline$C(11)-F e(1)-C(5)-C(4)$ & $-130.7(9)$ \\
\hline $\mathrm{N}(1)-\mathrm{Fe}(1)-\mathrm{C}(5)-\mathrm{C}(4)$ & $120.4(11)$ \\
\hline $\mathrm{C}(3)-\mathrm{Fe}(1)-\mathrm{C}(5)-\mathrm{C}(4)$ & $37.8(8)$ \\
\hline $\mathrm{B}(1)-\mathrm{Fe}(1)-\mathrm{C}(5)-\mathrm{C}(4)$ & $82.0(9)$ \\
\hline $\operatorname{Si}(1)-\mathrm{Fe}(1)-\mathrm{C}(5)-\mathrm{C}(4)$ & $-44.6(9)$ \\
\hline $\mathrm{C}(10)-\mathrm{Fe}(1)-\mathrm{C}(5)-\mathrm{N}(1)$ & $-9.0(15)$ \\
\hline $\mathrm{C}(11)-\mathrm{Fe}(1)-\mathrm{C}(5)-\mathrm{N}(1)$ & $108.9(7)$ \\
\hline $\mathrm{C}(4)-\mathrm{Fe}(1)-\mathrm{C}(5)-\mathrm{N}(1)$ & $-120.4(11)$ \\
\hline$C(3)-\mathrm{Fe}(1)-\mathrm{C}(5)-\mathrm{N}(1)$ & $-82.5(6)$ \\
\hline $\mathrm{B}(1)-\mathrm{Fe}(1)-\mathrm{C}(5)-\mathrm{N}(1)$ & $-38.4(6)$ \\
\hline $\operatorname{Si}(1)-\mathrm{Fe}(1)-\mathrm{C}(5)-\mathrm{N}(1)$ & $-165.0(5)$ \\
\hline $\mathrm{C}(5)-\mathrm{N}(1)-\mathrm{C}(6)-\mathrm{C}(9)$ & $147.9(10)$ \\
\hline $\mathrm{B}(1)-\mathrm{N}(1)-\mathrm{C}(6)-\mathrm{C}(9)$ & $-47.5(15)$ \\
\hline $\mathrm{Fe}(1)-\mathrm{N}(1)-\mathrm{C}(6)-\mathrm{C}(9)$ & $61.8(12)$ \\
\hline$C(5)-N(1)-C(6)-C(7)$ & $25.7(15)$ \\
\hline $\mathrm{B}(1)-\mathrm{N}(1)-\mathrm{C}(6)-\mathrm{C}(7)$ & $-169.7(13)$ \\
\hline $\mathrm{Fe}(1)-\mathrm{N}(1)-\mathrm{C}(6)-\mathrm{C}(7)$ & $-60.4(14)$ \\
\hline $\mathrm{C}(5)-\mathrm{N}(1)-\mathrm{C}(6)-\mathrm{C}(8)$ & $-91.6(11)$ \\
\hline $\mathrm{B}(1)-\mathrm{N}(1)-\mathrm{C}(6)-\mathrm{C}(8)$ & $73.0(14)$ \\
\hline $\mathrm{Fe}(1)-\mathrm{N}(1)-\mathrm{C}(6)-\mathrm{C}(8)$ & $-177.7(7)$ \\
\hline $\mathrm{C}(10)-\mathrm{Fe}(1)-\mathrm{C}(3)-\mathrm{C}(4)$ & $172.6(8)$ \\
\hline $\mathrm{C}(11)-\mathrm{Fe}(1)-\mathrm{C}(3)-\mathrm{C}(4)$ & $1.7(17)$ \\
\hline$C(5)-F e(1)-C(3)-C(4)$ & $-35.0(7)$ \\
\hline $\mathrm{N}(1)-\mathrm{Fe}(1)-\mathrm{C}(3)-\mathrm{C}(4)$ & $-77.8(8)$ \\
\hline $\mathrm{B}(1)-\mathrm{Fe}(1)-\mathrm{C}(3)-\mathrm{C}(4)$ & $-111.2(11)$ \\
\hline $\operatorname{Si}(1)-\mathrm{Fe}(1)-\mathrm{C}(3)-\mathrm{C}(4)$ & $85.5(7)$ \\
\hline $\mathrm{C}(10)-\mathrm{Fe}(1)-\mathrm{C}(3)-\mathrm{B}(1)$ & $-76.2(10)$ \\
\hline $\mathrm{C}(11)-\mathrm{Fe}(1)-\mathrm{C}(3)-\mathrm{B}(1)$ & $112.8(15)$ \\
\hline $\mathrm{C}(5)-\mathrm{Fe}(1)-\mathrm{C}(3)-\mathrm{B}(1)$ & $76.2(8)$ \\
\hline $\mathrm{C}(4)-\mathrm{Fe}(1)-\mathrm{C}(3)-\mathrm{B}(1)$ & $111.2(11)$ \\
\hline $\mathrm{N}(1)-\mathrm{Fe}(1)-\mathrm{C}(3)-\mathrm{B}(1)$ & $33.4(8)$ \\
\hline $\operatorname{Si}(1)-\mathrm{Fe}(1)-\mathrm{C}(3)-\mathrm{B}(1)$ & $-163.3(8)$ \\
\hline $\mathrm{C}(30)-\mathrm{Fe}(2)-\mathrm{C}(31)-\mathrm{O}(6)$ & $32(18)$ \\
\hline $\mathrm{C}(25)-\mathrm{Fe}(2)-\mathrm{C}(31)-\mathrm{O}(6)$ & $-129(17)$ \\
\hline $\mathrm{C}(24)-\mathrm{Fe}(2)-\mathrm{C}(31)-\mathrm{O}(6)$ & $-161(17)$ \\
\hline $\mathrm{N}(2)-\mathrm{Fe}(2)-\mathrm{C}(31)-\mathrm{O}(6)$ & $-89(18)$ \\
\hline $\mathrm{C}(23)-\mathrm{Fe}(2)-\mathrm{C}(31)-\mathrm{O}(6)$ & $-153(17)$ \\
\hline $\mathrm{B}(2)-\mathrm{Fe}(2)-\mathrm{C}(31)-\mathrm{O}(6)$ & $-69(18)$ \\
\hline
\end{tabular}




\begin{tabular}{|c|c|}
\hline $\mathrm{Si}(2)-\mathrm{Fe}(2)-\mathrm{C}(31)-\mathrm{O}(6)$ & 116(18) \\
\hline$N(1)-C(5)-C(4)-C(3)$ & $2.0(14)$ \\
\hline $\mathrm{Fe}(1)-\mathrm{C}(5)-\mathrm{C}(4)-\mathrm{C}(3)$ & $-60.4(8)$ \\
\hline$N(1)-C(5)-C(4)-F e(1)$ & $62.5(8)$ \\
\hline$B(1)-C(3)-C(4)-C(5)$ & $-8.1(13)$ \\
\hline $\mathrm{Fe}(1)-\mathrm{C}(3)-\mathrm{C}(4)-\mathrm{C}(5)$ & $59.9(9)$ \\
\hline $\mathrm{B}(1)-\mathrm{C}(3)-\mathrm{C}(4)-\mathrm{Fe}(1)$ & $-68.0(8)$ \\
\hline$C(10)-F e(1)-C(4)-C(5)$ & $-132.6(10)$ \\
\hline$C(11)-F e(1)-C(4)-C(5)$ & $60.3(9)$ \\
\hline $\mathrm{N}(1)-\mathrm{Fe}(1)-\mathrm{C}(4)-\mathrm{C}(5)$ & $-36.9(7)$ \\
\hline$C(3)-F e(1)-C(4)-C(5)$ & $-120.4(11)$ \\
\hline $\mathrm{B}(1)-\mathrm{Fe}(1)-\mathrm{C}(4)-\mathrm{C}(5)$ & $-78.8(8)$ \\
\hline $\operatorname{Si}(1)-\mathrm{Fe}(1)-\mathrm{C}(4)-\mathrm{C}(5)$ & $142.6(8)$ \\
\hline $\mathrm{C}(10)-\mathrm{Fe}(1)-\mathrm{C}(4)-\mathrm{C}(3)$ & $-12.2(13)$ \\
\hline $\mathrm{C}(11)-\mathrm{Fe}(1)-\mathrm{C}(4)-\mathrm{C}(3)$ & $-179.4(7)$ \\
\hline $\mathrm{C}(5)-\mathrm{Fe}(1)-\mathrm{C}(4)-\mathrm{C}(3)$ & $120.4(11)$ \\
\hline $\mathrm{N}(1)-\mathrm{Fe}(1)-\mathrm{C}(4)-\mathrm{C}(3)$ & $83.4(7)$ \\
\hline $\mathrm{B}(1)-\mathrm{Fe}(1)-\mathrm{C}(4)-\mathrm{C}(3)$ & $41.5(7)$ \\
\hline $\operatorname{Si}(1)-\mathrm{Fe}(1)-\mathrm{C}(4)-\mathrm{C}(3)$ & $-97.0(7)$ \\
\hline $\mathrm{C}(5)-\mathrm{N}(1)-\mathrm{B}(1)-\mathrm{O}(1)$ & $174.3(11)$ \\
\hline $\mathrm{C}(6)-\mathrm{N}(1)-\mathrm{B}(1)-\mathrm{O}(1)$ & $8.1(19)$ \\
\hline $\mathrm{Fe}(1)-\mathrm{N}(1)-\mathrm{B}(1)-\mathrm{O}(1)$ & $-124.0(12)$ \\
\hline $\mathrm{C}(5)-\mathrm{N}(1)-\mathrm{B}(1)-\mathrm{C}(3)$ & $-10.2(12)$ \\
\hline $\mathrm{C}(6)-\mathrm{N}(1)-\mathrm{B}(1)-\mathrm{C}(3)$ & $-176.4(10)$ \\
\hline $\mathrm{Fe}(1)-\mathrm{N}(1)-\mathrm{B}(1)-\mathrm{C}(3)$ & $51.4(8)$ \\
\hline $\mathrm{C}(5)-\mathrm{N}(1)-\mathrm{B}(1)-\mathrm{Fe}(1)$ & $-61.6(6)$ \\
\hline $\mathrm{C}(6)-\mathrm{N}(1)-\mathrm{B}(1)-\mathrm{Fe}(1)$ & $132.2(11)$ \\
\hline $\mathrm{C}(4)-\mathrm{C}(3)-\mathrm{B}(1)-\mathrm{O}(1)$ & $-174.0(13)$ \\
\hline $\mathrm{Fe}(1)-\mathrm{C}(3)-\mathrm{B}(1)-\mathrm{O}(1)$ & $123.2(14)$ \\
\hline $\mathrm{C}(4)-\mathrm{C}(3)-\mathrm{B}(1)-\mathrm{N}(1)$ & $11.0(13)$ \\
\hline $\mathrm{Fe}(1)-\mathrm{C}(3)-\mathrm{B}(1)-\mathrm{N}(1)$ & $-51.8(8)$ \\
\hline $\mathrm{C}(4)-\mathrm{C}(3)-\mathrm{B}(1)-\mathrm{Fe}(1)$ & $62.8(7)$ \\
\hline $\mathrm{C}(10)-\mathrm{Fe}(1)-\mathrm{B}(1)-\mathrm{O}(1)$ & $-13.3(12)$ \\
\hline $\mathrm{C}(11)-\mathrm{Fe}(1)-\mathrm{B}(1)-\mathrm{O}(1)$ & $88.6(15)$ \\
\hline $\mathrm{C}(5)-\mathrm{Fe}(1)-\mathrm{B}(1)-\mathrm{O}(1)$ & $153.4(14)$ \\
\hline $\mathrm{C}(4)-\mathrm{Fe}(1)-\mathrm{B}(1)-\mathrm{O}(1)$ & $-164.7(15)$ \\
\hline $\mathrm{N}(1)-\mathrm{Fe}(1)-\mathrm{B}(1)-\mathrm{O}(1)$ & $113.6(15)$ \\
\hline $\mathrm{C}(3)-\mathrm{Fe}(1)-\mathrm{B}(1)-\mathrm{O}(1)$ & $-122.4(17)$ \\
\hline $\mathrm{Si}(1)-\mathrm{Fe}(1)-\mathrm{B}(1)-\mathrm{O}(1)$ & $-99.2(12)$ \\
\hline $\mathrm{C}(10)-\mathrm{Fe}(1)-\mathrm{B}(1)-\mathrm{N}(1)$ & $-126.8(7)$ \\
\hline $\mathrm{C}(11)-\mathrm{Fe}(1)-\mathrm{B}(1)-\mathrm{N}(1)$ & $-24.9(12)$ \\
\hline $\mathrm{C}(5)-\mathrm{Fe}(1)-\mathrm{B}(1)-\mathrm{N}(1)$ & $39.9(7)$ \\
\hline $\mathrm{C}(4)-\mathrm{Fe}(1)-\mathrm{B}(1)-\mathrm{N}(1)$ & $81.8(8)$ \\
\hline $\mathrm{C}(3)-\mathrm{Fe}(1)-\mathrm{B}(1)-\mathrm{N}(1)$ & $124.1(12)$ \\
\hline $\operatorname{Si}(1)-\mathrm{Fe}(1)-\mathrm{B}(1)-\mathrm{N}(1)$ & $147.3(5)$ \\
\hline $\mathrm{C}(10)-\mathrm{Fe}(1)-\mathrm{B}(1)-\mathrm{C}(3)$ & 109.1(9) \\
\hline$C(11)-F e(1)-B(1)-C(3)$ & $-149.0(9)$ \\
\hline
\end{tabular}


$\mathrm{C}(5)-\mathrm{Fe}(1)-\mathrm{B}(1)-\mathrm{C}(3)$

$\mathrm{C}(4)-\mathrm{Fe}(1)-\mathrm{B}(1)-\mathrm{C}(3)$

$\mathrm{N}(1)-\mathrm{Fe}(1)-\mathrm{B}(1)-\mathrm{C}(3)$

$\mathrm{Si}(1)-\mathrm{Fe}(1)-\mathrm{B}(1)-\mathrm{C}(3)$

$\mathrm{C}(50)-\mathrm{O}(7)-\mathrm{C}(53)-\mathrm{C}(52)$

$\mathrm{C}(52)-\mathrm{C}(51)-\mathrm{C}(50)-\mathrm{O}(7)$

$\mathrm{C}(53)-\mathrm{O}(7)-\mathrm{C}(50)-\mathrm{C}(51)$

$\mathrm{C}(50)-\mathrm{C}(51)-\mathrm{C}(52)-\mathrm{C}(53)$

$\mathrm{O}(7)-\mathrm{C}(53)-\mathrm{C}(52)-\mathrm{C}(51)$
$-84.2(9)$

$-42.3(8)$

$-124.1(12)$

23.2(11)

1(5)

$-2(8)$

1(7)

$3(8)$

$-3(7)$ 


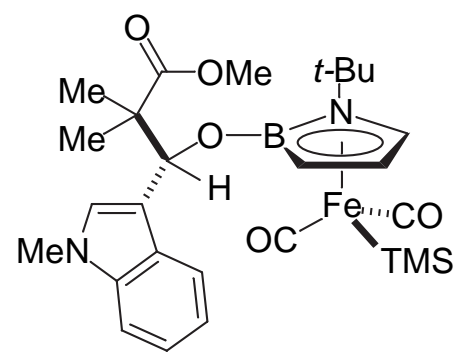

A yellow pentane/benzene solution was prepared. Crystals suitable for X-ray structural analysis were obtained by solvent evaporation at $-30^{\circ} \mathrm{C}$.

A yellow needle of dimensions $0.4 \times 0.21 \times 0.19 \mathrm{~mm}^{3}$ was mounted under STP and transferred to a Bruker AXS/CCD three-circle diffractometer equipped with a cold stream of $\mathrm{N}_{2}$ gas. An initial unit cell was determined with monochromated $\mathrm{Mo} \mathrm{K}_{\alpha}$ radiation $(\lambda=0.71073 \AA)$. The cell thus determined was triclinic.

A hemisphere of data was then collected using $\omega$ scans of $0.30^{\circ}$ and 30 -s frames. The raw data frames were integrated using the Bruker program SAINT+ for NT version 6.01. The data that were collected ( 6475 total reflections, 4531 unique, $R_{\text {int }}=0.0303$ ) had the following Miller index ranges: -11 to 11 in $h,-6$ to 12 in $k$, and -15 to 15 in 1 . A SADABS absorption correction was performed, with $\mathrm{T}_{\min }=0.700093$ and $\mathrm{T}_{\max }=$ 0.926385 .

All aspects of the solution and refinement were handled by SHELXTL NT version 5.10. The structure was solved by direct methods in the triclinic space group $\mathrm{P}-1, \mathrm{a}=$ 10.5502(9) $\AA$; $b=11.3979(9) \AA ; c=14.2293(12) \AA ; \alpha=96.9350(10)^{\circ} ; \beta=92.9950(10)^{\circ} ; \gamma=$ $107.6400(10)^{\circ}$, and refined using standard difference Fourier techniques. Final, fullmatrix least-squares refinement (4531 data for 345 parameters) on F2 yielded residuals of R1 and wR2 of 0.0676 and 0.1767 for data $\mathrm{I}>2 \sigma(\mathrm{I})$, and 0.0728 and 0.1802 , respectively, for all data. During the final refinement all non-hydrogen atoms were treated anisotropically. Hydrogen atoms were included in calculated positions and refined isotropically on a riding model. No extinction coefficient was used in the refinement. Residual electron density amounted to a maximum of $0.704 \mathrm{e} / \AA^{-3}$ and a minimum of $-0.760 \mathrm{e} / \AA^{-3}$.

Tables 1-6 provide the full crystallographic data for the X-ray structure. 


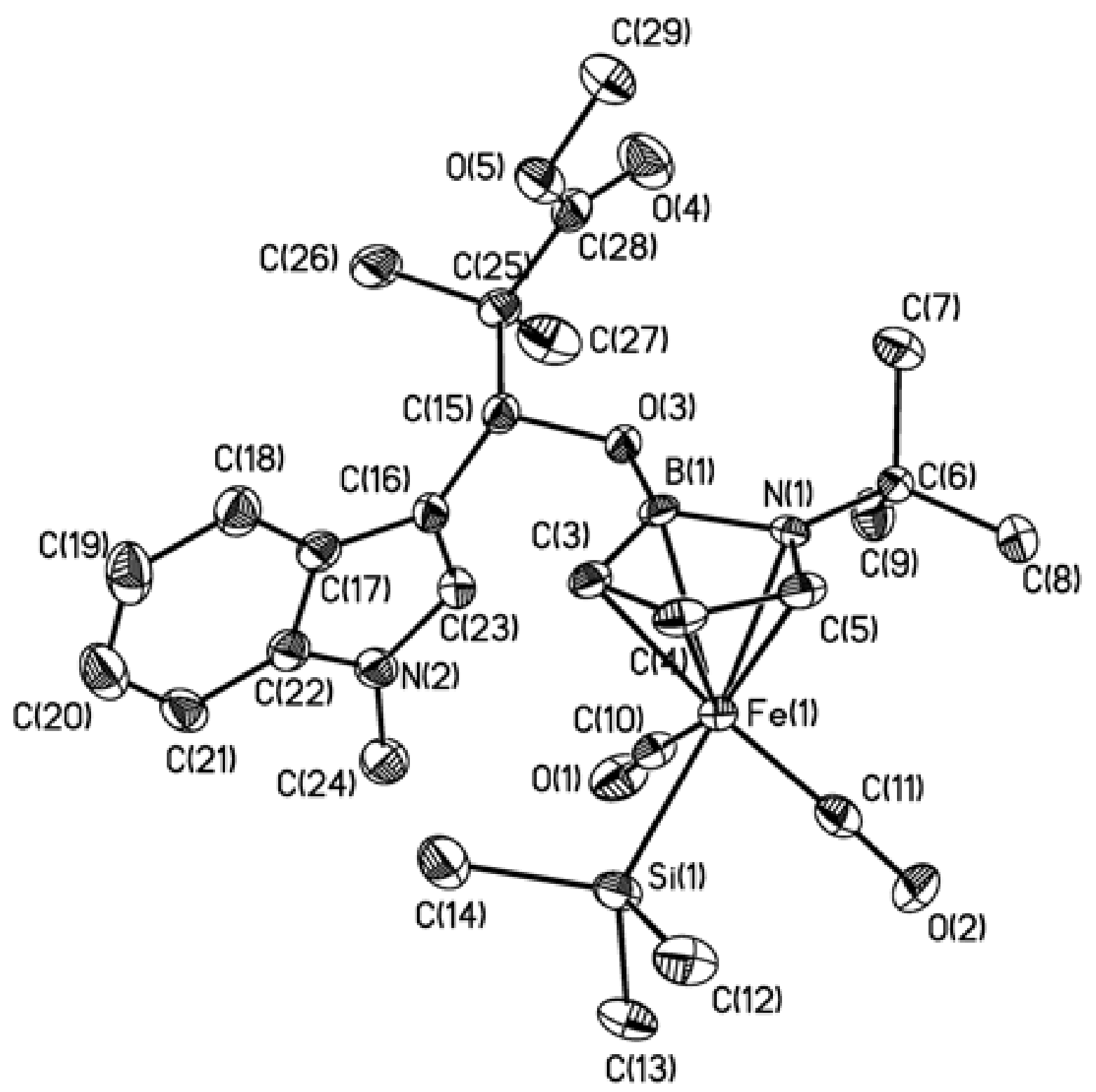


Table 1. Crystal data and structure refinement for 02145SLS.

Identification code

Empirical formula

Formula weight

Temperature

Wavelength

Crystal system

Space group

Unit cell dimensions

Volume

Z

Density (calculated)

Absorption coefficient

$\mathrm{F}(000)$

Crystal size

Theta range for data collection

Index ranges

Reflections collected

Independent reflections

Completeness to theta $=23.26^{\circ}$

Refinement method

Data / restraints / parameters

Goodness-of-fit on $\mathrm{F}^{2}$

Final $R$ indices [I>2sigma(I)]

$\mathrm{R}$ indices (all data)

Extinction coefficient

Largest diff. peak and hole 02145sls

C27 H39 B Fe N2 O5 Si

566.35

193(2) K

$0.71073 \AA$

Triclinic

P-1

$\mathrm{a}=10.5502(9) \AA \quad \mathrm{a}=96.9350(10)^{\circ}$.

$\mathrm{b}=11.3979(9) \AA \quad \mathrm{b}=92.9950(10)^{\circ}$.

$\mathrm{c}=14.2293(12) \AA \quad \mathrm{g}=107.6400(10)^{\circ}$.

$1611.5(2) \AA^{3}$

2

$1.167 \mathrm{Mg} / \mathrm{m}^{3}$

$0.539 \mathrm{~mm}^{-1}$

600

$0.4 \times 0.21 \times 0.19 \mathrm{~mm}^{3}$

2.38 to $23.26^{\circ}$.

$-11<=\mathrm{h}<=11,-6<=\mathrm{k}<=12,-15<=1<=15$

6475

$4531[\mathrm{R}(\mathrm{int})=0.0303]$

$97.8 \%$

Full-matrix least-squares on $\mathrm{F}^{2}$

4531 / 0 / 345

1.145

$\mathrm{R} 1=0.0676, \mathrm{wR} 2=0.1767$

$\mathrm{R} 1=0.0728, \mathrm{wR} 2=0.1802$

$0.0011(12)$

0.704 and -0.760 e. $\AA^{-3}$ 
Table 2. Atomic coordinates ( x 104) and equivalent isotropic displacement parameters $\left(\AA^{2} \times 10^{3}\right)$ for 02145SLS. U(eq) is defined as one third of the trace of the orthogonalized $\mathrm{U}^{\mathrm{ij}}$ tensor.

\begin{tabular}{|c|c|c|c|c|}
\hline & $x$ & $\mathrm{y}$ & $\mathrm{z}$ & $\mathrm{U}(\mathrm{eq})$ \\
\hline $\mathrm{Fe}(1)$ & $4121(1)$ & $934(1)$ & $2169(1)$ & $26(1)$ \\
\hline $\operatorname{Si}(1)$ & $4036(1)$ & $-750(1)$ & 2964(1) & $35(1)$ \\
\hline $\mathrm{O}(1)$ & $5823(4)$ & $-18(3)$ & $946(3)$ & $53(1)$ \\
\hline $\mathrm{O}(2)$ & $1724(4)$ & $-854(4)$ & 1151(3) & $60(1)$ \\
\hline $\mathrm{O}(3)$ & 6593(3) & $3640(3)$ & $1904(2)$ & $29(1)$ \\
\hline $\mathrm{O}(4)$ & $7872(5)$ & $6701(4)$ & $1522(3)$ & $65(1)$ \\
\hline $\mathrm{O}(5)$ & $7930(4)$ & $6372(3)$ & $3023(3)$ & $47(1)$ \\
\hline $\mathrm{N}(1)$ & $4135(4)$ & $2777(3)$ & 2024(3) & $27(1)$ \\
\hline $\mathrm{N}(2)$ & $8899(4)$ & $1126(4)$ & $1763(3)$ & $39(1)$ \\
\hline $\mathrm{B}(1)$ & $5544(5)$ & $2973(4)$ & $2361(4)$ & $28(1)$ \\
\hline$C(5)$ & $3335(5)$ & $2260(4)$ & $2725(3)$ & $31(1)$ \\
\hline$C(4)$ & $4119(5)$ & $2026(4)$ & $3455(3)$ & $34(1)$ \\
\hline$C(3)$ & $5451(5)$ & $2325(4)$ & $3234(3)$ & $29(1)$ \\
\hline$C(6)$ & $3606(4)$ & $3237(4)$ & 1185(3) & $32(1)$ \\
\hline$C(7)$ & $4005(6)$ & $4646(5)$ & $1423(4)$ & $50(1)$ \\
\hline$C(8)$ & 2098(5) & $2697(5)$ & $1004(4)$ & $47(1)$ \\
\hline$C(9)$ & $4243(5)$ & $2869(5)$ & $317(4)$ & $45(1)$ \\
\hline$C(10)$ & $5180(5)$ & $387(4)$ & $1438(4)$ & $36(1)$ \\
\hline$C(11)$ & $2675(5)$ & $-115(5)$ & $1563(4)$ & $39(1)$ \\
\hline$C(12)$ & $2576(6)$ & $-1153(5)$ & $3683(4)$ & $50(1)$ \\
\hline$C(13)$ & $3897(6)$ & $-2224(5)$ & $2147(4)$ & $51(1)$ \\
\hline$C(14)$ & $5583(6)$ & $-441(5)$ & $3790(4)$ & $51(1)$ \\
\hline$C(15)$ & $7923(4)$ & $3963(4)$ & $2374(3)$ & $31(1)$ \\
\hline$C(16)$ & $8443(4)$ & $2881(4)$ & $2304(3)$ & $31(1)$ \\
\hline$C(17)$ & $9075(5)$ & $2495(4)$ & $3080(4)$ & $35(1)$ \\
\hline$C(18)$ & $9433(5)$ & $2954(5)$ & $4047(4)$ & $44(1)$ \\
\hline$C(19)$ & $10042(6)$ & $2333(6)$ & $4597(4)$ & $54(2)$ \\
\hline$C(20)$ & $10316(6)$ & $1269(6)$ & $4217(5)$ & $56(2)$ \\
\hline$C(21)$ & $9972(5)$ & $769(5)$ & $3272(4)$ & $48(1)$ \\
\hline$C(22)$ & $9347(5)$ & $1397(5)$ & $2716(4)$ & $37(1)$ \\
\hline$C(23)$ & $8372(5)$ & $2020(4)$ & $1537(3)$ & $35(1)$ \\
\hline$C(24)$ & $8857(6)$ & $-7(5)$ & $1132(4)$ & $53(2)$ \\
\hline$C(25)$ & $8773(5)$ & $5098(4)$ & $1947(4)$ & $36(1)$ \\
\hline$C(26)$ & $10180(5)$ & $5558(5)$ & $2474(5)$ & $58(2)$ \\
\hline$C(27)$ & $8822(7)$ & $4784(6)$ & $881(5)$ & $61(2)$ \\
\hline$C(28)$ & $8142(5)$ & $6138(5)$ & $2112(4)$ & $40(1)$ \\
\hline$C(29)$ & $7344(6)$ & $7354(6)$ & $3253(5)$ & $59(2)$ \\
\hline
\end{tabular}


Table 3. Bond lengths $[\AA]$ and angles $\left[^{\circ}\right]$ for 02145 SLS.

\begin{tabular}{|c|c|}
\hline $\mathrm{Fe}(1)-\mathrm{C}(11)$ & $1.734(5)$ \\
\hline $\mathrm{Fe}(1)-\mathrm{C}(10)$ & $1.762(5)$ \\
\hline $\mathrm{Fe}(1)-\mathrm{C}(5)$ & $2.034(4)$ \\
\hline $\mathrm{Fe}(1)-\mathrm{C}(4)$ & $2.087(5)$ \\
\hline $\mathrm{Fe}(1)-\mathrm{N}(1)$ & $2.131(3)$ \\
\hline $\mathrm{Fe}(1)-\mathrm{C}(3)$ & $2.152(4)$ \\
\hline $\mathrm{Fe}(1)-\mathrm{Si}(1)$ & $2.3244(14)$ \\
\hline $\mathrm{Fe}(1)-\mathrm{B}(1)$ & $2.328(5)$ \\
\hline $\operatorname{Si}(1)-C(12)$ & $1.865(6)$ \\
\hline $\operatorname{Si}(1)-C(14)$ & $1.876(6)$ \\
\hline $\operatorname{Si}(1)-C(13)$ & $1.886(5)$ \\
\hline $\mathrm{O}(1)-\mathrm{C}(10)$ & $1.151(6)$ \\
\hline $\mathrm{O}(2)-\mathrm{C}(11)$ & $1.170(6)$ \\
\hline $\mathrm{O}(3)-\mathrm{B}(1)$ & $1.383(6)$ \\
\hline $\mathrm{O}(3)-\mathrm{C}(15)$ & $1.442(5)$ \\
\hline $\mathrm{O}(4)-\mathrm{C}(28)$ & $1.189(6)$ \\
\hline $\mathrm{O}(5)-\mathrm{C}(28)$ & $1.337(7)$ \\
\hline $\mathrm{O}(5)-\mathrm{C}(29)$ & $1.447(6)$ \\
\hline $\mathrm{N}(1)-\mathrm{C}(5)$ & $1.408(6)$ \\
\hline $\mathrm{N}(1)-\mathrm{B}(1)$ & $1.480(6)$ \\
\hline $\mathrm{N}(1)-\mathrm{C}(6)$ & $1.507(6)$ \\
\hline $\mathrm{N}(2)-\mathrm{C}(23)$ & $1.361(6)$ \\
\hline $\mathrm{N}(2)-\mathrm{C}(22)$ & $1.379(7)$ \\
\hline $\mathrm{N}(2)-\mathrm{C}(24)$ & $1.469(7)$ \\
\hline $\mathrm{B}(1)-\mathrm{C}(3)$ & $1.513(7)$ \\
\hline$C(5)-C(4)$ & $1.394(7)$ \\
\hline$C(4)-C(3)$ & $1.404(7)$ \\
\hline$C(6)-C(9)$ & $1.512(7)$ \\
\hline$C(6)-C(8)$ & $1.517(7)$ \\
\hline$C(6)-C(7)$ & $1.521(7)$ \\
\hline$C(15)-C(16)$ & $1.489(7)$ \\
\hline$C(15)-C(25)$ & $1.546(6)$ \\
\hline$C(16)-C(23)$ & $1.359(7)$ \\
\hline$C(16)-C(17)$ & $1.441(7)$ \\
\hline C(17)-C(18) & $1.400(7)$ \\
\hline$C(17)-C(22)$ & $1.411(7)$ \\
\hline $\mathrm{C}(18)-\mathrm{C}(19)$ & $1.373(8)$ \\
\hline$C(19)-C(20)$ & $1.384(9)$ \\
\hline$C(20)-C(21)$ & $1.379(9)$ \\
\hline$C(21)-C(22)$ & $1.394(7)$ \\
\hline$C(25)-C(27)$ & $1.523(8)$ \\
\hline$C(25)-C(28)$ & $1.527(7)$ \\
\hline$C(25)-C(26)$ & $1.536(7)$ \\
\hline $\mathrm{C}(11)-\mathrm{Fe}(1)-\mathrm{C}(10)$ & $93.6(2)$ \\
\hline
\end{tabular}




$\begin{array}{ll}\mathrm{C}(11)-\mathrm{Fe}(1)-\mathrm{C}(5) & 98.7(2) \\ \mathrm{C}(10)-\mathrm{Fe}(1)-\mathrm{C}(5) & 153.3(2) \\ \mathrm{C}(11)-\mathrm{Fe}(1)-\mathrm{C}(4) & 122.6(2) \\ \mathrm{C}(10)-\mathrm{Fe}(1)-\mathrm{C}(4) & 142.5(2) \\ \mathrm{C}(5)-\mathrm{Fe}(1)-\mathrm{C}(4) & 39.53(19) \\ \mathrm{C}(11)-\mathrm{Fe}(1)-\mathrm{N}(1) & 109.08(19) \\ \mathrm{C}(10)-\mathrm{Fe}(1)-\mathrm{N}(1) & 113.99(19) \\ \mathrm{C}(5)-\mathrm{Fe}(1)-\mathrm{N}(1) & 39.44(16) \\ \mathrm{C}(4)-\mathrm{Fe}(1)-\mathrm{N}(1) & 66.12(17) \\ \mathrm{C}(11)-\mathrm{Fe}(1)-\mathrm{C}(3) & 161.3(2) \\ \mathrm{C}(10)-\mathrm{Fe}(1)-\mathrm{C}(3) & 104.76(19) \\ \mathrm{C}(5)-\mathrm{Fe}(1)-\mathrm{C}(3) & 66.16(18) \\ \mathrm{C}(4)-\mathrm{Fe}(1)-\mathrm{C}(3) & 38.64(18) \\ \mathrm{N}(1)-\mathrm{Fe}(1)-\mathrm{C}(3) & 66.79(16) \\ \mathrm{C}(11)-\mathrm{Fe}(1)-\mathrm{Si}(1) & 81.66(17) \\ \mathrm{C}(10)-\mathrm{Fe}(1)-\mathrm{Si}(1) & 85.41(16) \\ \mathrm{C}(5)-\mathrm{Fe}(1)-\mathrm{Si}(1) & 119.69(14) \\ \mathrm{C}(4)-\mathrm{Fe}(1)-\mathrm{Si}(1) & 90.27(14) \\ \mathrm{N}(1)-\mathrm{Fe}(1)-\mathrm{Si}(1) & 156.33(11) \\ \mathrm{C}(3)-\mathrm{Fe}(1)-\mathrm{Si}(1) & 96.11(13) \\ \mathrm{C}(11)-\mathrm{Fe}(1)-\mathrm{B}(1) & 145.2(2) \\ \mathrm{C}(10)-\mathrm{Fe}(1)-\mathrm{B}(1) & 92.5(2) \\ \mathrm{C}(5)-\mathrm{Fe}(1)-\mathrm{B}(1) & 64.23(18) \\ \mathrm{C}(4)-\mathrm{Fe}(1)-\mathrm{B}(1) & 63.99(18) \\ \mathrm{N}(1)-\mathrm{Fe}(1)-\mathrm{B}(1) & 38.44(16) \\ \mathrm{C}(3)-\mathrm{Fe}(1)-\mathrm{B}(1) & 39.23(18) \\ \mathrm{Si}(1)-\mathrm{Fe}(1)-\mathrm{B}(1) & 132.97(14) \\ \mathrm{C}(12)-\mathrm{Si}(1)-\mathrm{C}(14) & 107.5(3) \\ \mathrm{C}(12)-\mathrm{Si}(1)-\mathrm{C}(13) & 105.5(3) \\ \mathrm{C}(14)-\mathrm{Si}(1)-\mathrm{C}(13) & 105.7(3) \\ \mathrm{C}(12)-\mathrm{Si}(1)-\mathrm{Fe}(1) & 112.55(19) \\ \mathrm{C}(14)-\mathrm{Si}(1)-\mathrm{Fe}(1) & 111.54(19) \\ \mathrm{C}(13)-\mathrm{Si}(1)-\mathrm{Fe}(1) & 113.61(19) \\ \mathrm{B}(1)-\mathrm{O}(3)-\mathrm{C}(15) & 117.7(3) \\ \mathrm{C}(28)-\mathrm{O}(5)-\mathrm{C}(29) & 116.0(4) \\ \mathrm{C}(5)-\mathrm{N}(1)-\mathrm{B}(1) & 107.7(4) \\ \mathrm{C}(5)-\mathrm{N}(1)-\mathrm{C}(6) & 123.4(4) \\ \mathrm{B}(1)-\mathrm{N}(1)-\mathrm{C}(6) & 128.1(4)-\mathrm{N}(1)-\mathrm{C}(3) \\ \mathrm{C}(5)-\mathrm{N}(1)-\mathrm{Fe}(1) & 66.6(2) \\ \mathrm{B}(1)-\mathrm{N}(1)-\mathrm{Fe}(1) & 78.0(2) \\ \mathrm{C}(6)-\mathrm{N}(1)-\mathrm{Fe}(1) & 128.6(3) \\ \mathrm{C}(23)-\mathrm{N}(2)-\mathrm{C}(22) & 108.1(4) \\ & 125.9(4) \\ & 125.6(4) \\ \mathrm{C}(23) & 121.9(4) \\ & \end{array}$




$\begin{array}{ll}\mathrm{O}(3)-\mathrm{B}(1)-\mathrm{Fe}(1) & 136.1(3) \\ \mathrm{N}(1)-\mathrm{B}(1)-\mathrm{Fe}(1) & 63.5(2) \\ \mathrm{C}(3)-\mathrm{B}(1)-\mathrm{Fe}(1) & 64.1(2) \\ \mathrm{C}(4)-\mathrm{C}(5)-\mathrm{N}(1) & 110.4(4) \\ \mathrm{C}(4)-\mathrm{C}(5)-\mathrm{Fe}(1) & 72.3(3) \\ \mathrm{N}(1)-\mathrm{C}(5)-\mathrm{Fe}(1) & 74.0(2) \\ \mathrm{C}(5)-\mathrm{C}(4)-\mathrm{C}(3) & 109.7(4) \\ \mathrm{C}(5)-\mathrm{C}(4)-\mathrm{Fe}(1) & 68.2(3) \\ \mathrm{C}(3)-\mathrm{C}(4)-\mathrm{Fe}(1) & 73.2(3) \\ \mathrm{C}(4)-\mathrm{C}(3)-\mathrm{B}(1) & 107.2(4) \\ \mathrm{C}(4)-\mathrm{C}(3)-\mathrm{Fe}(1) & 68.1(3) \\ \mathrm{B}(1)-\mathrm{C}(3)-\mathrm{Fe}(1) & 76.7(3) \\ \mathrm{N}(1)-\mathrm{C}(6)-\mathrm{C}(9) & 108.5(4) \\ \mathrm{N}(1)-\mathrm{C}(6)-\mathrm{C}(8) & 111.6(4) \\ \mathrm{C}(9)-\mathrm{C}(6)-\mathrm{C}(8) & 109.5(4) \\ \mathrm{N}(1)-\mathrm{C}(6)-\mathrm{C}(7) & 106.6(4) \\ \mathrm{C}(9)-\mathrm{C}(6)-\mathrm{C}(7) & 110.7(4) \\ \mathrm{C}(8)-\mathrm{C}(6)-\mathrm{C}(7) & 109.9(4) \\ \mathrm{O}(1)-\mathrm{C}(10)-\mathrm{Fe}(1) & 176.9(4) \\ \mathrm{O}(2)-\mathrm{C}(11)-\mathrm{Fe}(1) & 177.6(4) \\ \mathrm{O}(3)-\mathrm{C}(15)-\mathrm{C}(16) & 112.0(4) \\ \mathrm{O}(3)-\mathrm{C}(15)-\mathrm{C}(25) & 105.9(4) \\ \mathrm{C}(16)-\mathrm{C}(15)-\mathrm{C}(25) & 115.1(4) \\ \mathrm{C}(23)-\mathrm{C}(16)-\mathrm{C}(17) & 105.4(4) \\ \mathrm{C}(23)-\mathrm{C}(16)-\mathrm{C}(15) & 128.9(4) \\ \mathrm{C}(17)-\mathrm{C}(16)-\mathrm{C}(15) & 125.7(4) \\ \mathrm{C}(18)-\mathrm{C}(17)-\mathrm{C}(22) & 118.1(5) \\ \mathrm{C}(18)-\mathrm{C}(17)-\mathrm{C}(16) & 134.7(5) \\ \mathrm{C}(22)-\mathrm{C}(17)-\mathrm{C}(16) & 107.2(4) \\ \mathrm{C}(19)-\mathrm{C}(18)-\mathrm{C}(17) & 119.1(5) \\ \mathrm{C}(18)-\mathrm{C}(19)-\mathrm{C}(20) & 121.5(6) \\ \mathrm{C}(21)-\mathrm{C}(20)-\mathrm{C}(19) & 121.8(5) \\ \mathrm{C}(20)-\mathrm{C}(21)-\mathrm{C}(22) & 116.6(5) \\ \mathrm{N}(2)-\mathrm{C}(22)-\mathrm{C}(21) & 129.6(5) \\ \mathrm{N}(2)-\mathrm{C}(22)-\mathrm{C}(17) & 107.5(4) \\ \mathrm{C}(21)-\mathrm{C}(22)-\mathrm{C}(17) & 122.9(5) \\ \mathrm{C}(16)-\mathrm{C}(23)-\mathrm{N}(2) & 111.8(4) \\ \mathrm{C}(27)-\mathrm{C}(25)-\mathrm{C}(28) & 108.3(4) \\ \mathrm{C}(27)-\mathrm{C}(25)-\mathrm{C}(26) & 111.3(5) \\ \mathrm{C}(28)-\mathrm{C}(25)-\mathrm{C}(26) & 108.2(4) \\ \mathrm{C}(27)-\mathrm{C}(25)-\mathrm{C}(15) & 111.5(4) \\ \mathrm{C}(28)-\mathrm{C}(25)-\mathrm{C}(15) & 108.6(4) \\ \mathrm{C}(26)-\mathrm{C}(25)-\mathrm{C}(15) & 108.7(4) \\ \mathrm{O}(4)-\mathrm{C}(28)-\mathrm{O}(5) & 122.4(5) \\ \mathrm{O}(4)-\mathrm{C}(28)-\mathrm{C}(25) & 125.8(5) \\ \mathrm{O}(5)-\mathrm{C}(28)-\mathrm{C}(25) & 11.8(4) \\ & \end{array}$


Table 4. Anisotropic displacement parameters $\left(\AA^{2} \times 10^{3}\right)$ for 02145SLS. The anisotropic displacement factor exponent takes the form: $-2 p^{2}\left[h^{2} a^{* 2} U^{11}+\ldots+2 h k a^{*} b^{*} U^{12}\right]$

\begin{tabular}{|c|c|c|c|c|c|c|}
\hline & $\mathrm{U}^{11}$ & $\mathrm{U}^{22}$ & $\mathrm{U}^{33}$ & $\mathrm{U}^{23}$ & $\mathrm{U}^{13}$ & $\mathrm{U}^{12}$ \\
\hline $\mathrm{Fe}(1)$ & $26(1)$ & $20(1)$ & $31(1)$ & $3(1)$ & $1(1)$ & $6(1)$ \\
\hline $\operatorname{Si}(1)$ & $41(1)$ & $25(1)$ & $42(1)$ & $9(1)$ & $6(1)$ & $12(1)$ \\
\hline $\mathrm{O}(1)$ & $47(2)$ & $41(2)$ & $64(3)$ & $-15(2)$ & $17(2)$ & $11(2)$ \\
\hline $\mathrm{O}(2)$ & $41(2)$ & $39(2)$ & $88(3)$ & $-4(2)$ & $-21(2)$ & $4(2)$ \\
\hline $\mathrm{O}(3)$ & $24(2)$ & $27(2)$ & $33(2)$ & $7(1)$ & $-1(1)$ & $4(1)$ \\
\hline $\mathrm{O}(4)$ & $78(3)$ & $60(3)$ & $66(3)$ & $26(2)$ & $2(2)$ & $30(2)$ \\
\hline $\mathrm{O}(5)$ & $53(2)$ & $39(2)$ & $53(2)$ & $6(2)$ & $1(2)$ & $23(2)$ \\
\hline $\mathrm{N}(1)$ & $28(2)$ & $17(2)$ & $36(2)$ & $5(2)$ & $2(2)$ & $8(2)$ \\
\hline $\mathrm{N}(2)$ & $34(2)$ & $32(2)$ & $52(3)$ & $3(2)$ & $5(2)$ & $14(2)$ \\
\hline $\mathrm{B}(1)$ & $25(3)$ & $18(2)$ & $36(3)$ & $-4(2)$ & $-1(2)$ & $3(2)$ \\
\hline$C(5)$ & $28(2)$ & $21(2)$ & $40(3)$ & $0(2)$ & $10(2)$ & $4(2)$ \\
\hline$C(4)$ & $39(3)$ & $23(2)$ & $35(3)$ & $-2(2)$ & $10(2)$ & $5(2)$ \\
\hline$C(3)$ & $32(2)$ & $21(2)$ & $30(2)$ & $-1(2)$ & $-2(2)$ & $3(2)$ \\
\hline$C(6)$ & $29(2)$ & $24(2)$ & $40(3)$ & $9(2)$ & $-7(2)$ & $5(2)$ \\
\hline$C(7)$ & $58(4)$ & $32(3)$ & $62(4)$ & $12(3)$ & $-6(3)$ & $17(3)$ \\
\hline$C(8)$ & $37(3)$ & $41(3)$ & $65(4)$ & $14(3)$ & $-8(3)$ & $14(2)$ \\
\hline$C(9)$ & $47(3)$ & $56(3)$ & $36(3)$ & $7(2)$ & $-2(2)$ & $20(3)$ \\
\hline$C(10)$ & $34(3)$ & $26(3)$ & $41(3)$ & $2(2)$ & $-3(2)$ & $1(2)$ \\
\hline$C(11)$ & $44(3)$ & $31(3)$ & $46(3)$ & $5(2)$ & $4(2)$ & $19(3)$ \\
\hline$C(12)$ & $58(4)$ & $39(3)$ & $57(3)$ & $13(3)$ & $14(3)$ & $15(3)$ \\
\hline$C(13)$ & $67(4)$ & $29(3)$ & $61(4)$ & $9(3)$ & $9(3)$ & $22(3)$ \\
\hline$C(14)$ & $59(4)$ & $47(3)$ & $51(3)$ & $11(3)$ & $-4(3)$ & $22(3)$ \\
\hline$C(15)$ & $25(2)$ & $31(3)$ & $33(2)$ & $7(2)$ & $0(2)$ & $5(2)$ \\
\hline$C(16)$ & $24(2)$ & $32(3)$ & $36(3)$ & $8(2)$ & $0(2)$ & $7(2)$ \\
\hline$C(17)$ & $26(2)$ & $32(3)$ & $43(3)$ & $9(2)$ & $7(2)$ & $2(2)$ \\
\hline$C(18)$ & $38(3)$ & $47(3)$ & $45(3)$ & $9(2)$ & $1(2)$ & $9(2)$ \\
\hline$C(19)$ & $47(3)$ & $72(4)$ & $45(3)$ & $25(3)$ & $0(3)$ & $15(3)$ \\
\hline$C(20)$ & $41(3)$ & $64(4)$ & $71(4)$ & $36(3)$ & $7(3)$ & $17(3)$ \\
\hline$C(21)$ & $32(3)$ & $40(3)$ & $75(4)$ & $25(3)$ & $7(3)$ & $11(2)$ \\
\hline$C(22)$ & $27(2)$ & $35(3)$ & $48(3)$ & $6(2)$ & $4(2)$ & $8(2)$ \\
\hline$C(23)$ & $31(3)$ & $34(3)$ & $39(3)$ & $7(2)$ & $-2(2)$ & $10(2)$ \\
\hline$C(24)$ & $46(3)$ & $44(3)$ & $71(4)$ & $-2(3)$ & $4(3)$ & $22(3)$ \\
\hline$C(25)$ & $28(2)$ & $30(3)$ & $46(3)$ & $11(2)$ & $6(2)$ & $2(2)$ \\
\hline$C(26)$ & $32(3)$ & $36(3)$ & $99(5)$ & $14(3)$ & $2(3)$ & $0(2)$ \\
\hline$C(27)$ & $71(4)$ & $54(4)$ & $68(4)$ & $27(3)$ & $36(3)$ & $20(3)$ \\
\hline$C(28)$ & $25(3)$ & $35(3)$ & $55(3)$ & $12(3)$ & $-3(2)$ & $1(2)$ \\
\hline$C(29)$ & $61(4)$ & $46(3)$ & $76(4)$ & $3(3)$ & $1(3)$ & $31(3)$ \\
\hline
\end{tabular}


Table 5. Hydrogen coordinates $\left(x 10^{4}\right)$ and isotropic displacement parameters $\left(\AA^{2} \times 10^{3}\right)$ for 02145SLS.

\begin{tabular}{|c|c|c|c|c|}
\hline & $\mathrm{x}$ & $\mathrm{y}$ & z & $\mathrm{U}(\mathrm{eq})$ \\
\hline $\mathrm{H}(5)$ & 2358 & 2148 & 2728 & 37 \\
\hline $\mathrm{H}(4)$ & 3785 & 1700 & 4047 & 40 \\
\hline $\mathrm{H}(3)$ & 6216 & 2226 & 3628 & 35 \\
\hline $\mathrm{H}(7 \mathrm{~A})$ & 3580 & 4867 & 1985 & 75 \\
\hline $\mathrm{H}(7 \mathrm{~B})$ & 3714 & 4991 & 884 & 75 \\
\hline $\mathrm{H}(7 \mathrm{C})$ & 4978 & 4988 & 1555 & 75 \\
\hline $\mathrm{H}(8 \mathrm{~A})$ & 1840 & 1787 & 933 & 71 \\
\hline $\mathrm{H}(8 \mathrm{~B})$ & 1797 & 2952 & 421 & 71 \\
\hline $\mathrm{H}(8 \mathrm{C})$ & 1684 & 3003 & 1542 & 71 \\
\hline $\mathrm{H}(9 \mathrm{~A})$ & 5217 & 3177 & 448 & 68 \\
\hline $\mathrm{H}(9 \mathrm{~B})$ & 3974 & 3230 & -221 & 68 \\
\hline $\mathrm{H}(9 \mathrm{C})$ & 3949 & 1960 & 162 & 68 \\
\hline $\mathrm{H}(12 \mathrm{~A})$ & 2598 & -1851 & 4017 & 75 \\
\hline $\mathrm{H}(12 \mathrm{~B})$ & 1747 & -1389 & 3262 & 75 \\
\hline $\mathrm{H}(12 \mathrm{C})$ & 2616 & -431 & 4147 & 75 \\
\hline $\mathrm{H}(13 \mathrm{~A})$ & 3849 & -2898 & 2525 & 76 \\
\hline $\mathrm{H}(13 \mathrm{~B})$ & 4682 & -2093 & 1786 & 76 \\
\hline $\mathrm{H}(13 \mathrm{C})$ & 3088 & -2448 & 1707 & 76 \\
\hline $\mathrm{H}(14 \mathrm{~A})$ & 5670 & 282 & 4268 & 77 \\
\hline $\mathrm{H}(14 \mathrm{~B})$ & 6367 & -272 & 3427 & 77 \\
\hline $\mathrm{H}(14 \mathrm{C})$ & 5520 & -1170 & 4108 & 77 \\
\hline $\mathrm{H}(15)$ & 7881 & 4238 & 3063 & 37 \\
\hline $\mathrm{H}(18)$ & 9256 & 3685 & 4318 & 53 \\
\hline $\mathrm{H}(19)$ & 10282 & 2640 & 5253 & 65 \\
\hline $\mathrm{H}(20)$ & 10752 & 872 & 4617 & 67 \\
\hline $\mathrm{H}(21)$ & 10152 & 34 & 3014 & 57 \\
\hline $\mathrm{H}(23)$ & 8000 & 2039 & 919 & 42 \\
\hline $\mathrm{H}(24 \mathrm{~A})$ & 8238 & -732 & 1349 & 79 \\
\hline $\mathrm{H}(24 \mathrm{~B})$ & 9752 & -97 & 1143 & 79 \\
\hline $\mathrm{H}(24 \mathrm{C})$ & 8552 & 51 & 482 & 79 \\
\hline $\mathrm{H}(26 \mathrm{~A})$ & 10689 & 6345 & 2273 & 87 \\
\hline $\mathrm{H}(26 \mathrm{~B})$ & 10637 & 4936 & 2324 & 87 \\
\hline $\mathrm{H}(26 \mathrm{C})$ & 10113 & 5686 & 3161 & 87 \\
\hline $\mathrm{H}(27 \mathrm{~A})$ & 7911 & 4417 & 573 & 92 \\
\hline $\mathrm{H}(27 \mathrm{~B})$ & 9319 & 4188 & 766 & 92 \\
\hline $\mathrm{H}(27 \mathrm{C})$ & 9269 & 5544 & 620 & 92 \\
\hline$H(29 A)$ & 7918 & 8130 & 3073 & 88 \\
\hline $\mathrm{H}(29 B)$ & 7259 & 7469 & 3938 & 88 \\
\hline $\mathrm{H}(29 \mathrm{C})$ & 6458 & 7128 & 2905 & 88 \\
\hline
\end{tabular}


Table 6. Torsion angles $\left[{ }^{\circ}\right]$ for $02145 S L S$.

\begin{tabular}{|c|c|}
\hline$C(11)-F e(1)-S i(1)-C(12)$ & $-66.5(3)$ \\
\hline$C(10)-F e(1)-S i(1)-C(12)$ & $-160.9(3)$ \\
\hline $\mathrm{C}(5)-\mathrm{Fe}(1)-\mathrm{Si}(1)-\mathrm{C}(12)$ & $28.7(3)$ \\
\hline$C(4)-F e(1)-S i(1)-C(12)$ & $56.5(3)$ \\
\hline $\mathrm{N}(1)-\mathrm{Fe}(1)-\mathrm{Si}(1)-\mathrm{C}(12)$ & $52.7(3)$ \\
\hline$C(3)-F e(1)-S i(1)-C(12)$ & $94.7(2)$ \\
\hline $\mathrm{B}(1)-\mathrm{Fe}(1)-\mathrm{Si}(1)-\mathrm{C}(12)$ & $110.0(3)$ \\
\hline$C(11)-F e(1)-S i(1)-C(14)$ & $172.6(3)$ \\
\hline$C(10)-F e(1)-S i(1)-C(14)$ & $78.2(3)$ \\
\hline $\mathrm{C}(5)-\mathrm{Fe}(1)-\mathrm{Si}(1)-\mathrm{C}(14)$ & $-92.2(3)$ \\
\hline$C(4)-F e(1)-S i(1)-C(14)$ & $-64.5(2)$ \\
\hline $\mathrm{N}(1)-\mathrm{Fe}(1)-\mathrm{Si}(1)-\mathrm{C}(14)$ & $-68.2(3)$ \\
\hline$C(3)-F e(1)-S i(1)-C(14)$ & $-26.2(2)$ \\
\hline $\mathrm{B}(1)-\mathrm{Fe}(1)-\mathrm{Si}(1)-\mathrm{C}(14)$ & $-11.0(3)$ \\
\hline$C(11)-F e(1)-S i(1)-C(13)$ & $53.3(3)$ \\
\hline$C(10)-F e(1)-S i(1)-C(13)$ & $-41.1(3)$ \\
\hline$C(5)-F e(1)-S i(1)-C(13)$ & $148.5(3)$ \\
\hline$C(4)-F e(1)-S i(1)-C(13)$ & 176.2(3) \\
\hline $\mathrm{N}(1)-\mathrm{Fe}(1)-\mathrm{Si}(1)-\mathrm{C}(13)$ & $172.5(3)$ \\
\hline$C(3)-F e(1)-S i(1)-C(13)$ & $-145.5(2)$ \\
\hline $\mathrm{B}(1)-\mathrm{Fe}(1)-\mathrm{Si}(1)-\mathrm{C}(13)$ & $-130.3(3)$ \\
\hline $\mathrm{C}(11)-\mathrm{Fe}(1)-\mathrm{N}(1)-\mathrm{C}(5)$ & $80.2(3)$ \\
\hline$C(10)-F e(1)-N(1)-C(5)$ & $-176.6(3)$ \\
\hline $\mathrm{C}(4)-\mathrm{Fe}(1)-\mathrm{N}(1)-\mathrm{C}(5)$ & $-37.9(3)$ \\
\hline $\mathrm{C}(3)-\mathrm{Fe}(1)-\mathrm{N}(1)-\mathrm{C}(5)$ & $-80.2(3)$ \\
\hline $\operatorname{Si}(1)-\mathrm{Fe}(1)-\mathrm{N}(1)-\mathrm{C}(5)$ & $-33.8(4)$ \\
\hline $\mathrm{B}(1)-\mathrm{Fe}(1)-\mathrm{N}(1)-\mathrm{C}(5)$ & $-115.5(4)$ \\
\hline $\mathrm{C}(11)-\mathrm{Fe}(1)-\mathrm{N}(1)-\mathrm{B}(1)$ & $-164.2(3)$ \\
\hline $\mathrm{C}(10)-\mathrm{Fe}(1)-\mathrm{N}(1)-\mathrm{B}(1)$ & $-61.1(3)$ \\
\hline $\mathrm{C}(5)-\mathrm{Fe}(1)-\mathrm{N}(1)-\mathrm{B}(1)$ & $115.5(4)$ \\
\hline $\mathrm{C}(4)-\mathrm{Fe}(1)-\mathrm{N}(1)-\mathrm{B}(1)$ & $77.7(3)$ \\
\hline $\mathrm{C}(3)-\mathrm{Fe}(1)-\mathrm{N}(1)-\mathrm{B}(1)$ & $35.4(3)$ \\
\hline $\mathrm{Si}(1)-\mathrm{Fe}(1)-\mathrm{N}(1)-\mathrm{B}(1)$ & $81.8(4)$ \\
\hline$C(11)-F e(1)-N(1)-C(6)$ & $-34.7(4)$ \\
\hline$C(10)-F e(1)-N(1)-C(6)$ & $68.5(4)$ \\
\hline$C(5)-F e(1)-N(1)-C(6)$ & $-114.9(4)$ \\
\hline$C(4)-\mathrm{Fe}(1)-\mathrm{N}(1)-\mathrm{C}(6)$ & $-152.7(4)$ \\
\hline$C(3)-F e(1)-N(1)-C(6)$ & $165.0(4)$ \\
\hline $\mathrm{Si}(1)-\mathrm{Fe}(1)-\mathrm{N}(1)-\mathrm{C}(6)$ & $-148.7(3)$ \\
\hline $\mathrm{B}(1)-\mathrm{Fe}(1)-\mathrm{N}(1)-\mathrm{C}(6)$ & $129.6(5)$ \\
\hline $\mathrm{C}(15)-\mathrm{O}(3)-\mathrm{B}(1)-\mathrm{N}(1)$ & $169.6(4)$ \\
\hline $\mathrm{C}(15)-\mathrm{O}(3)-\mathrm{B}(1)-\mathrm{C}(3)$ & $-9.2(7)$ \\
\hline $\mathrm{C}(15)-\mathrm{O}(3)-\mathrm{B}(1)-\mathrm{Fe}(1)$ & $-106.7(5)$ \\
\hline $\mathrm{C}(5)-\mathrm{N}(1)-\mathrm{B}(1)-\mathrm{O}(3)$ & $-170.0(4)$ \\
\hline $\mathrm{C}(6)-\mathrm{N}(1)-\mathrm{B}(1)-\mathrm{O}(3)$ & $-0.5(7)$ \\
\hline
\end{tabular}




\begin{tabular}{|c|c|}
\hline $\mathrm{Fe}(1)-\mathrm{N}(1)-\mathrm{B}(1)-\mathrm{O}(3)$ & $129.6(4)$ \\
\hline$C(5)-N(1)-B(1)-C(3)$ & $9.1(4)$ \\
\hline $\mathrm{C}(6)-\mathrm{N}(1)-\mathrm{B}(1)-\mathrm{C}(3)$ & $178.7(4)$ \\
\hline $\mathrm{Fe}(1)-\mathrm{N}(1)-\mathrm{B}(1)-\mathrm{C}(3)$ & $-51.2(3)$ \\
\hline $\mathrm{C}(5)-\mathrm{N}(1)-\mathrm{B}(1)-\mathrm{Fe}(1)$ & $60.4(3)$ \\
\hline $\mathrm{C}(6)-\mathrm{N}(1)-\mathrm{B}(1)-\mathrm{Fe}(1)$ & $-130.1(4)$ \\
\hline $\mathrm{C}(11)-\mathrm{Fe}(1)-\mathrm{B}(1)-\mathrm{O}(3)$ & $-82.8(6)$ \\
\hline $\mathrm{C}(10)-\mathrm{Fe}(1)-\mathrm{B}(1)-\mathrm{O}(3)$ & $17.2(5)$ \\
\hline $\mathrm{C}(5)-\mathrm{Fe}(1)-\mathrm{B}(1)-\mathrm{O}(3)$ & $-149.1(5)$ \\
\hline $\mathrm{C}(4)-\mathrm{Fe}(1)-\mathrm{B}(1)-\mathrm{O}(3)$ & $166.7(6)$ \\
\hline $\mathrm{N}(1)-\mathrm{Fe}(1)-\mathrm{B}(1)-\mathrm{O}(3)$ & $-109.6(6)$ \\
\hline $\mathrm{C}(3)-\mathrm{Fe}(1)-\mathrm{B}(1)-\mathrm{O}(3)$ & $127.7(6)$ \\
\hline $\mathrm{Si}(1)-\mathrm{Fe}(1)-\mathrm{B}(1)-\mathrm{O}(3)$ & $103.3(5)$ \\
\hline $\mathrm{C}(11)-\mathrm{Fe}(1)-\mathrm{B}(1)-\mathrm{N}(1)$ & $26.7(5)$ \\
\hline $\mathrm{C}(10)-\mathrm{Fe}(1)-\mathrm{B}(1)-\mathrm{N}(1)$ & $126.8(3)$ \\
\hline $\mathrm{C}(5)-\mathrm{Fe}(1)-\mathrm{B}(1)-\mathrm{N}(1)$ & $-39.5(2)$ \\
\hline $\mathrm{C}(4)-\mathrm{Fe}(1)-\mathrm{B}(1)-\mathrm{N}(1)$ & $-83.7(3)$ \\
\hline $\mathrm{C}(3)-\mathrm{Fe}(1)-\mathrm{B}(1)-\mathrm{N}(1)$ & $-122.7(4)$ \\
\hline $\mathrm{Si}(1)-\mathrm{Fe}(1)-\mathrm{B}(1)-\mathrm{N}(1)$ & $-147.12(19)$ \\
\hline $\mathrm{C}(11)-\mathrm{Fe}(1)-\mathrm{B}(1)-\mathrm{C}(3)$ & $149.5(4)$ \\
\hline $\mathrm{C}(10)-\mathrm{Fe}(1)-\mathrm{B}(1)-\mathrm{C}(3)$ & $-110.5(3)$ \\
\hline $\mathrm{C}(5)-\mathrm{Fe}(1)-\mathrm{B}(1)-\mathrm{C}(3)$ & $83.2(3)$ \\
\hline $\mathrm{C}(4)-\mathrm{Fe}(1)-\mathrm{B}(1)-\mathrm{C}(3)$ & $39.0(3)$ \\
\hline $\mathrm{N}(1)-\mathrm{Fe}(1)-\mathrm{B}(1)-\mathrm{C}(3)$ & $122.7(4)$ \\
\hline $\operatorname{Si}(1)-\mathrm{Fe}(1)-\mathrm{B}(1)-\mathrm{C}(3)$ & $-24.4(3)$ \\
\hline $\mathrm{B}(1)-\mathrm{N}(1)-\mathrm{C}(5)-\mathrm{C}(4)$ & $-4.3(5)$ \\
\hline $\mathrm{C}(6)-\mathrm{N}(1)-\mathrm{C}(5)-\mathrm{C}(4)$ & $-174.5(4)$ \\
\hline $\mathrm{Fe}(1)-\mathrm{N}(1)-\mathrm{C}(5)-\mathrm{C}(4)$ & $63.6(3)$ \\
\hline $\mathrm{B}(1)-\mathrm{N}(1)-\mathrm{C}(5)-\mathrm{Fe}(1)$ & $-67.9(3)$ \\
\hline $\mathrm{C}(6)-\mathrm{N}(1)-\mathrm{C}(5)-\mathrm{Fe}(1)$ & $121.9(4)$ \\
\hline$C(11)-F e(1)-C(5)-C(4)$ & $132.2(3)$ \\
\hline$C(10)-F e(1)-C(5)-C(4)$ & $-111.3(5)$ \\
\hline $\mathrm{N}(1)-\mathrm{Fe}(1)-\mathrm{C}(5)-\mathrm{C}(4)$ & $-118.2(4)$ \\
\hline $\mathrm{C}(3)-\mathrm{Fe}(1)-\mathrm{C}(5)-\mathrm{C}(4)$ & $-36.3(3)$ \\
\hline $\operatorname{Si}(1)-\mathrm{Fe}(1)-\mathrm{C}(5)-\mathrm{C}(4)$ & $47.0(3)$ \\
\hline B(1)-Fe(1)-C(5)-C(4) & $-79.6(3)$ \\
\hline $\mathrm{C}(11)-\mathrm{Fe}(1)-\mathrm{C}(5)-\mathrm{N}(1)$ & $-109.6(3)$ \\
\hline $\mathrm{C}(10)-\mathrm{Fe}(1)-\mathrm{C}(5)-\mathrm{N}(1)$ & $6.9(6)$ \\
\hline $\mathrm{C}(4)-\mathrm{Fe}(1)-\mathrm{C}(5)-\mathrm{N}(1)$ & $118.2(4)$ \\
\hline $\mathrm{C}(3)-\mathrm{Fe}(1)-\mathrm{C}(5)-\mathrm{N}(1)$ & $81.9(3)$ \\
\hline $\operatorname{Si}(1)-\mathrm{Fe}(1)-\mathrm{C}(5)-\mathrm{N}(1)$ & 165.12(19) \\
\hline $\mathrm{B}(1)-\mathrm{Fe}(1)-\mathrm{C}(5)-\mathrm{N}(1)$ & $38.5(2)$ \\
\hline $\mathrm{N}(1)-\mathrm{C}(5)-\mathrm{C}(4)-\mathrm{C}(3)$ & $-2.9(5)$ \\
\hline $\mathrm{Fe}(1)-\mathrm{C}(5)-\mathrm{C}(4)-\mathrm{C}(3)$ & $61.8(3)$ \\
\hline$N(1)-C(5)-C(4)-F e(1)$ & $-64.7(3)$ \\
\hline$C(11)-F e(1)-C(4)-C(5)$ & $-60.3(3)$ \\
\hline$C(10)-F e(1)-C(4)-C(5)$ & $136.6(4)$ \\
\hline
\end{tabular}




\begin{tabular}{|c|c|}
\hline $\mathrm{N}(1)-\mathrm{Fe}(1)-\mathrm{C}(4)-\mathrm{C}(5)$ & $37.8(3)$ \\
\hline$C(3)-F e(1)-C(4)-C(5)$ & $119.9(4)$ \\
\hline $\operatorname{Si}(1)-\mathrm{Fe}(1)-\mathrm{C}(4)-\mathrm{C}(5)$ & $-140.6(3)$ \\
\hline $\mathrm{B}(1)-\mathrm{Fe}(1)-\mathrm{C}(4)-\mathrm{C}(5)$ & $80.3(3)$ \\
\hline$C(11)-F e(1)-C(4)-C(3)$ & 179.7(3) \\
\hline $\mathrm{C}(10)-\mathrm{Fe}(1)-\mathrm{C}(4)-\mathrm{C}(3)$ & $16.7(5)$ \\
\hline $\mathrm{C}(5)-\mathrm{Fe}(1)-\mathrm{C}(4)-\mathrm{C}(3)$ & $-119.9(4)$ \\
\hline $\mathrm{N}(1)-\mathrm{Fe}(1)-\mathrm{C}(4)-\mathrm{C}(3)$ & $-82.2(3)$ \\
\hline $\operatorname{Si}(1)-\mathrm{Fe}(1)-\mathrm{C}(4)-\mathrm{C}(3)$ & $99.5(3)$ \\
\hline $\mathrm{B}(1)-\mathrm{Fe}(1)-\mathrm{C}(4)-\mathrm{C}(3)$ & $-39.6(3)$ \\
\hline$C(5)-C(4)-C(3)-B(1)$ & $8.7(5)$ \\
\hline $\mathrm{Fe}(1)-\mathrm{C}(4)-\mathrm{C}(3)-\mathrm{B}(1)$ & $67.4(3)$ \\
\hline$C(5)-C(4)-C(3)-F e(1)$ & $-58.7(3)$ \\
\hline $\mathrm{O}(3)-\mathrm{B}(1)-\mathrm{C}(3)-\mathrm{C}(4)$ & $168.1(5)$ \\
\hline $\mathrm{N}(1)-\mathrm{B}(1)-\mathrm{C}(3)-\mathrm{C}(4)$ & $-10.8(5)$ \\
\hline $\mathrm{Fe}(1)-\mathrm{B}(1)-\mathrm{C}(3)-\mathrm{C}(4)$ & $-61.7(3)$ \\
\hline $\mathrm{O}(3)-\mathrm{B}(1)-\mathrm{C}(3)-\mathrm{Fe}(1)$ & $-130.1(5)$ \\
\hline $\mathrm{N}(1)-\mathrm{B}(1)-\mathrm{C}(3)-\mathrm{Fe}(1)$ & $50.9(3)$ \\
\hline $\mathrm{C}(11)-\mathrm{Fe}(1)-\mathrm{C}(3)-\mathrm{C}(4)$ & $-0.7(7)$ \\
\hline $\mathrm{C}(10)-\mathrm{Fe}(1)-\mathrm{C}(3)-\mathrm{C}(4)$ & $-169.6(3)$ \\
\hline $\mathrm{C}(5)-\mathrm{Fe}(1)-\mathrm{C}(3)-\mathrm{C}(4)$ & $37.1(3)$ \\
\hline $\mathrm{N}(1)-\mathrm{Fe}(1)-\mathrm{C}(3)-\mathrm{C}(4)$ & $80.3(3)$ \\
\hline $\operatorname{Si}(1)-\mathrm{Fe}(1)-\mathrm{C}(3)-\mathrm{C}(4)$ & $-82.7(3)$ \\
\hline $\mathrm{B}(1)-\mathrm{Fe}(1)-\mathrm{C}(3)-\mathrm{C}(4)$ & $114.9(4)$ \\
\hline $\mathrm{C}(11)-\mathrm{Fe}(1)-\mathrm{C}(3)-\mathrm{B}(1)$ & $-115.6(6)$ \\
\hline $\mathrm{C}(10)-\mathrm{Fe}(1)-\mathrm{C}(3)-\mathrm{B}(1)$ & $75.5(3)$ \\
\hline $\mathrm{C}(5)-\mathrm{Fe}(1)-\mathrm{C}(3)-\mathrm{B}(1)$ & $-77.9(3)$ \\
\hline $\mathrm{C}(4)-\mathrm{Fe}(1)-\mathrm{C}(3)-\mathrm{B}(1)$ & $-114.9(4)$ \\
\hline $\mathrm{N}(1)-\mathrm{Fe}(1)-\mathrm{C}(3)-\mathrm{B}(1)$ & $-34.7(3)$ \\
\hline $\operatorname{Si}(1)-\mathrm{Fe}(1)-\mathrm{C}(3)-\mathrm{B}(1)$ & $162.3(2)$ \\
\hline $\mathrm{C}(5)-\mathrm{N}(1)-\mathrm{C}(6)-\mathrm{C}(9)$ & $-140.1(4)$ \\
\hline $\mathrm{B}(1)-\mathrm{N}(1)-\mathrm{C}(6)-\mathrm{C}(9)$ & $51.9(6)$ \\
\hline $\mathrm{Fe}(1)-\mathrm{N}(1)-\mathrm{C}(6)-\mathrm{C}(9)$ & $-54.9(5)$ \\
\hline $\mathrm{C}(5)-\mathrm{N}(1)-\mathrm{C}(6)-\mathrm{C}(8)$ & $-19.3(6)$ \\
\hline $\mathrm{B}(1)-\mathrm{N}(1)-\mathrm{C}(6)-\mathrm{C}(8)$ & $172.7(4)$ \\
\hline $\mathrm{Fe}(1)-\mathrm{N}(1)-\mathrm{C}(6)-\mathrm{C}(8)$ & $65.9(5)$ \\
\hline $\mathrm{C}(5)-\mathrm{N}(1)-\mathrm{C}(6)-\mathrm{C}(7)$ & $100.6(5)$ \\
\hline $\mathrm{B}(1)-\mathrm{N}(1)-\mathrm{C}(6)-\mathrm{C}(7)$ & $-67.4(6)$ \\
\hline $\mathrm{Fe}(1)-\mathrm{N}(1)-\mathrm{C}(6)-\mathrm{C}(7)$ & $-174.1(3)$ \\
\hline $\mathrm{C}(11)-\mathrm{Fe}(1)-\mathrm{C}(10)-\mathrm{O}(1)$ & $-15(9)$ \\
\hline $\mathrm{C}(5)-\mathrm{Fe}(1)-\mathrm{C}(10)-\mathrm{O}(1)$ & $-132(8)$ \\
\hline $\mathrm{C}(4)-\mathrm{Fe}(1)-\mathrm{C}(10)-\mathrm{O}(1)$ & $151(8)$ \\
\hline $\mathrm{N}(1)-\mathrm{Fe}(1)-\mathrm{C}(10)-\mathrm{O}(1)$ & $-128(9)$ \\
\hline $\mathrm{C}(3)-\mathrm{Fe}(1)-\mathrm{C}(10)-\mathrm{O}(1)$ & $162(9)$ \\
\hline $\mathrm{Si}(1)-\mathrm{Fe}(1)-\mathrm{C}(10)-\mathrm{O}(1)$ & $66(9)$ \\
\hline $\mathrm{B}(1)-\mathrm{Fe}(1)-\mathrm{C}(10)-\mathrm{O}(1)$ & $-161(9)$ \\
\hline $\mathrm{C}(10)-\mathrm{Fe}(1)-\mathrm{C}(11)-\mathrm{O}(2)$ & $38(12)$ \\
\hline
\end{tabular}




\begin{tabular}{|c|c|}
\hline$C(5)-F e(1)-C(11)-O(2)$ & $-166(12)$ \\
\hline $\mathrm{C}(4)-\mathrm{Fe}(1)-\mathrm{C}(11)-\mathrm{O}(2)$ & $-132(12)$ \\
\hline $\mathrm{N}(1)-\mathrm{Fe}(1)-\mathrm{C}(11)-\mathrm{O}(2)$ & $155(12)$ \\
\hline $\mathrm{C}(3)-\mathrm{Fe}(1)-\mathrm{C}(11)-\mathrm{O}(2)$ & $-131(11)$ \\
\hline $\mathrm{Si}(1)-\mathrm{Fe}(1)-\mathrm{C}(11)-\mathrm{O}(2)$ & $-47(12)$ \\
\hline $\mathrm{B}(1)-\mathrm{Fe}(1)-\mathrm{C}(11)-\mathrm{O}(2)$ & $138(12)$ \\
\hline $\mathrm{B}(1)-\mathrm{O}(3)-\mathrm{C}(15)-\mathrm{C}(16)$ & $76.3(5)$ \\
\hline $\mathrm{B}(1)-\mathrm{O}(3)-\mathrm{C}(15)-\mathrm{C}(25)$ & $-157.5(4)$ \\
\hline $\mathrm{O}(3)-\mathrm{C}(15)-\mathrm{C}(16)-\mathrm{C}(23)$ & $43.8(6)$ \\
\hline$C(25)-C(15)-C(16)-C(23)$ & $-77.3(6)$ \\
\hline $\mathrm{O}(3)-\mathrm{C}(15)-\mathrm{C}(16)-\mathrm{C}(17)$ & $-132.3(4)$ \\
\hline$C(25)-C(15)-C(16)-C(17)$ & $106.6(5)$ \\
\hline$C(23)-C(16)-C(17)-C(18)$ & $-179.4(5)$ \\
\hline$C(15)-C(16)-C(17)-C(18)$ & $-2.6(8)$ \\
\hline$C(23)-C(16)-C(17)-C(22)$ & $-0.1(5)$ \\
\hline$C(15)-C(16)-C(17)-C(22)$ & $176.8(4)$ \\
\hline$C(22)-C(17)-C(18)-C(19)$ & $0.8(7)$ \\
\hline$C(16)-C(17)-C(18)-C(19)$ & $-179.9(5)$ \\
\hline$C(17)-C(18)-C(19)-C(20)$ & $0.3(8)$ \\
\hline$C(18)-C(19)-C(20)-C(21)$ & $-1.0(9)$ \\
\hline$C(19)-C(20)-C(21)-C(22)$ & $0.6(8)$ \\
\hline $\mathrm{C}(23)-\mathrm{N}(2)-\mathrm{C}(22)-\mathrm{C}(21)$ & $-179.2(5)$ \\
\hline $\mathrm{C}(24)-\mathrm{N}(2)-\mathrm{C}(22)-\mathrm{C}(21)$ & $7.3(8)$ \\
\hline$C(23)-N(2)-C(22)-C(17)$ & $0.2(5)$ \\
\hline $\mathrm{C}(24)-\mathrm{N}(2)-\mathrm{C}(22)-\mathrm{C}(17)$ & $-173.3(4)$ \\
\hline $\mathrm{C}(20)-\mathrm{C}(21)-\mathrm{C}(22)-\mathrm{N}(2)$ & $179.8(5)$ \\
\hline$C(20)-C(21)-C(22)-C(17)$ & $0.4(7)$ \\
\hline $\mathrm{C}(18)-\mathrm{C}(17)-\mathrm{C}(22)-\mathrm{N}(2)$ & $179.4(4)$ \\
\hline $\mathrm{C}(16)-\mathrm{C}(17)-\mathrm{C}(22)-\mathrm{N}(2)$ & $-0.1(5)$ \\
\hline$C(18)-C(17)-C(22)-C(21)$ & $-1.2(7)$ \\
\hline$C(16)-C(17)-C(22)-C(21)$ & $179.4(4)$ \\
\hline $\mathrm{C}(17)-\mathrm{C}(16)-\mathrm{C}(23)-\mathrm{N}(2)$ & $0.2(5)$ \\
\hline$C(15)-C(16)-C(23)-N(2)$ & $-176.5(4)$ \\
\hline$C(22)-N(2)-C(23)-C(16)$ & $-0.3(6)$ \\
\hline$C(24)-N(2)-C(23)-C(16)$ & $173.2(5)$ \\
\hline $\mathrm{O}(3)-\mathrm{C}(15)-\mathrm{C}(25)-\mathrm{C}(27)$ & $-61.3(5)$ \\
\hline$C(16)-C(15)-C(25)-C(27)$ & $63.0(6)$ \\
\hline $\mathrm{O}(3)-\mathrm{C}(15)-\mathrm{C}(25)-\mathrm{C}(28)$ & $58.0(5)$ \\
\hline$C(16)-C(15)-C(25)-C(28)$ & $-177.7(4)$ \\
\hline $\mathrm{O}(3)-\mathrm{C}(15)-\mathrm{C}(25)-\mathrm{C}(26)$ & $175.5(4)$ \\
\hline$C(16)-C(15)-C(25)-C(26)$ & $-60.1(6)$ \\
\hline $\mathrm{C}(29)-\mathrm{O}(5)-\mathrm{C}(28)-\mathrm{O}(4)$ & $0.2(7)$ \\
\hline $\mathrm{C}(29)-\mathrm{O}(5)-\mathrm{C}(28)-\mathrm{C}(25)$ & $179.5(4)$ \\
\hline $\mathrm{C}(27)-\mathrm{C}(25)-\mathrm{C}(28)-\mathrm{O}(4)$ & $-7.6(7)$ \\
\hline $\mathrm{C}(26)-\mathrm{C}(25)-\mathrm{C}(28)-\mathrm{O}(4)$ & $113.2(6)$ \\
\hline $\mathrm{C}(15)-\mathrm{C}(25)-\mathrm{C}(28)-\mathrm{O}(4)$ & $-128.9(5)$ \\
\hline $\mathrm{C}(27)-\mathrm{C}(25)-\mathrm{C}(28)-\mathrm{O}(5)$ & $173.2(4)$ \\
\hline
\end{tabular}


$\mathrm{C}(26)-\mathrm{C}(25)-\mathrm{C}(28)-\mathrm{O}(5)$

$-66.0(5)$

$\mathrm{C}(15)-\mathrm{C}(25)-\mathrm{C}(28)-\mathrm{O}(5)$

$51.9(5)$ 


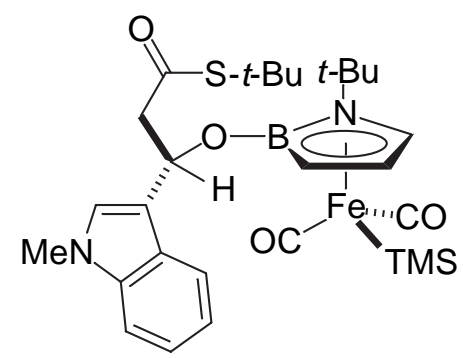

A yellow pentane/benzene $/ \mathrm{CH}_{2} \mathrm{Cl}_{2}$ solution was prepared. Crystals suitable for Xray structural analysis were obtained by solvent evaporation at $-30^{\circ} \mathrm{C}$.

A yellow block of dimensions $0.25 \times 0.2 \times 0.19 \mathrm{~mm}^{3}$ was mounted under STP and transferred to a Bruker AXS/CCD three-circle diffractometer equipped with a cold stream of $\mathrm{N}_{2}$ gas. An initial unit cell was determined with monochromated $\mathrm{Mo} \mathrm{K}_{\alpha}$ radiation $(\lambda=0.71073 \AA)$. The cell thus determined was monoclinic.

A hemisphere of data was then collected using $\omega$ scans of $0.30^{\circ}$ and $30-\mathrm{s}$ frames. The raw data frames were integrated using the Bruker program SAINT+ for NT version 6.01. The data that were collected (14741 total reflections, 4616 unique, $R_{\text {int }}=0.1062$ ) had the following Miller index ranges: -11 to 11 in h, -27 to 27 in $k$, and -10 to 13 in 1 . No absorption correction was performed.

All aspects of the solution and refinement were handled by SHELXTL NT version 5.10. The structure was solved by direct methods in the chiral monoclinic space group P2(1) $/ \mathrm{c}, \mathrm{a}=10.4994(11) \AA ; \mathrm{b}=24.850(3) \AA ; \mathrm{c}=12.3176(14) \AA ; \alpha=90^{\circ} ; \beta=92.295^{\circ} ; \gamma=90^{\circ}$, and refined using standard difference Fourier techniques. Final, full-matrix leastsquares refinement (4616 data for 353 parameters) on F2 yielded residuals of R1 and $\mathrm{wR} 2$ of 0.0439 and 0.1007 for data I > $2 \sigma(\mathrm{I})$, and 0.0737 and 0.1121 , respectively, for all data. During the final refinement all non-hydrogen atoms were treated anisotropically. Hydrogen atoms were included in calculated positions and refined isotropically on a riding model. No extinction coefficient was used in the refinement. Residual electron density amounted to a maximum of $0.443 \mathrm{e} / \AA^{-3}$ and a minimum of $-0.477 \mathrm{e} / \AA^{-3}$.

Tables 1-6 provide the full crystallographic data for the $\mathrm{X}$-ray structure. 


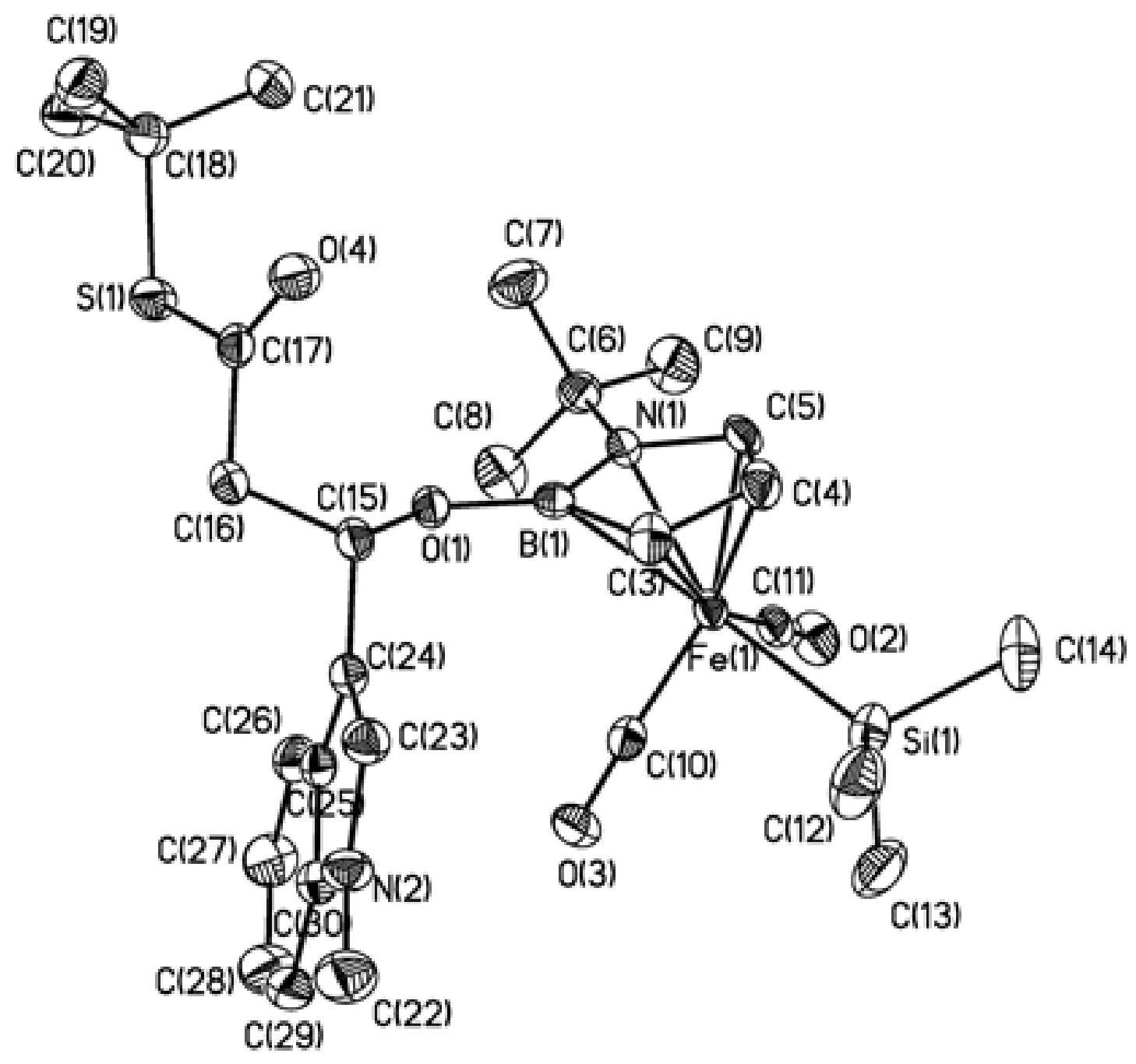


Table 1. Crystal data and structure refinement for 03211slm.

Identification code

Empirical formula

Formula weight

Temperature

Wavelength

Crystal system

Space group

Unit cell dimensions

Volume

Z

Density (calculated)

Absorption coefficient

$\mathrm{F}(000)$

Crystal size

Theta range for data collection

Index ranges

Reflections collected

Independent reflections

Completeness to theta $=23.28^{\circ}$

Refinement method

Data / restraints / parameters

Goodness-of-fit on $\mathrm{F}^{2}$

Final $\mathrm{R}$ indices [I>2sigma(I)]

$\mathrm{R}$ indices (all data)

Largest diff. peak and hole 03211slm

C28 H41 B Fe N2 O4 S Si

596.44

193(2) K

$0.71073 \AA$

Monoclinic

P2(1)/c

$\mathrm{a}=10.4994(11) \AA \quad \mathrm{a}=90^{\circ}$.

$\mathrm{b}=24.850(3) \AA$

$\mathrm{b}=92.295(2)^{\circ}$.

$\mathrm{c}=12.3176(14) \AA$

3211.2(6) $\AA^{3}$

4

$1.234 \mathrm{Mg} / \mathrm{m}^{3}$

$0.605 \mathrm{~mm}^{-1}$

1264

$0.25 \times 0.2 \times 0.19 \mathrm{~mm}^{3}$

1.64 to $23.28^{\circ}$.

$-11<=\mathrm{h}<=11,-27<=\mathrm{k}<=27,-10<=\mathrm{l}<=13$

14741

$4616[\mathrm{R}(\mathrm{int})=0.1062]$

$99.7 \%$

Full-matrix least-squares on $\mathrm{F}^{2}$

4616 / 0 / 353

0.924

$\mathrm{R} 1=0.0439, \mathrm{wR} 2=0.1007$

$\mathrm{R} 1=0.0737, \mathrm{wR} 2=0.1121$

0.443 and -0.477 e. $\AA^{-3}$ 
Table 2. Atomic coordinates $\left(x 10^{4}\right)$ and equivalent isotropic displacement parameters $\left(\AA^{2} \times 10^{3}\right)$ for $03211 \mathrm{slm}$. $\mathrm{U}(\mathrm{eq})$ is defined as one third of the trace of the orthogonalized $\mathrm{U}^{\mathrm{ij}}$ tensor.

\begin{tabular}{|c|c|c|c|c|}
\hline & $\mathrm{x}$ & $\mathrm{y}$ & $\mathrm{z}$ & $\mathrm{U}(\mathrm{eq})$ \\
\hline $\mathrm{Fe}(1)$ & $3858(1)$ & $6649(1)$ & $8178(1)$ & $28(1)$ \\
\hline$S(1)$ & $9834(1)$ & $5931(1)$ & $10939(1)$ & $39(1)$ \\
\hline $\operatorname{Si}(1)$ & $1845(1)$ & $6488(1)$ & $7387(1)$ & $43(1)$ \\
\hline $\mathrm{N}(1)$ & $5114(2)$ & 6795(1) & $9535(2)$ & $31(1)$ \\
\hline $\mathrm{N}(2)$ & $5643(3)$ & $4565(1)$ & $7016(2)$ & $37(1)$ \\
\hline $\mathrm{O}(1)$ & $6527(2)$ & $6013(1)$ & $9257(2)$ & $31(1)$ \\
\hline $\mathrm{O}(2)$ & $3493(2)$ & $7698(1)$ & $7189(2)$ & $53(1)$ \\
\hline $\mathrm{O}(3)$ & $4783(2)$ & 6113(1) & $6265(2)$ & $48(1)$ \\
\hline $\mathrm{O}(4)$ & $7600(2)$ & $5554(1)$ & $11539(2)$ & $44(1)$ \\
\hline $\mathrm{B}(1)$ & $5317(3)$ & $6221(2)$ & $9343(3)$ & $30(1)$ \\
\hline$C(3)$ & $3971(3)$ & $5984(2)$ & $9291(3)$ & $36(1)$ \\
\hline$C(4)$ & $3159(3)$ & $6393(2)$ & $9644(3)$ & $40(1)$ \\
\hline$C(5)$ & $3812(3)$ & $6874(2)$ & $9752(3)$ & $38(1)$ \\
\hline$C(6)$ & $6113(3)$ & $7226(2)$ & $9729(3)$ & $39(1)$ \\
\hline$C(7)$ & $6785(4)$ & $7096(2)$ & 10812(3) & $65(1)$ \\
\hline$C(8)$ & $7033(3)$ & $7198(2)$ & $8810(3)$ & $55(1)$ \\
\hline$C(9)$ & $5522(4)$ & $7783(2)$ & $9772(4)$ & $64(1)$ \\
\hline$C(10)$ & $4445(3)$ & $6323(2)$ & $7037(3)$ & $33(1)$ \\
\hline$C(11)$ & $3634(3)$ & $7277(2)$ & $7595(3)$ & $36(1)$ \\
\hline$C(12)$ & 1391(4) & $5767(2)$ & $7535(4)$ & $69(1)$ \\
\hline$C(13)$ & $1723(4)$ & $6641(2)$ & $5892(4)$ & $73(2)$ \\
\hline$C(14)$ & $572(3)$ & $6910(2)$ & $8017(4)$ & $69(1)$ \\
\hline$C(15)$ & $6711(3)$ & $5434(1)$ & $9296(3)$ & $30(1)$ \\
\hline$C(16)$ & 8099(3) & $5365(2)$ & $9699(3)$ & $35(1)$ \\
\hline$C(17)$ & $8346(3)$ & $5598(1)$ & $10814(3)$ & $31(1)$ \\
\hline$C(18)$ & 10130(3) & $6090(2)$ & 12391(3) & $37(1)$ \\
\hline$C(19)$ & 10163(3) & $5575(2)$ & $13056(3)$ & $48(1)$ \\
\hline$C(20)$ & $11457(3)$ & $6348(2)$ & $12422(3)$ & $61(1)$ \\
\hline$C(21)$ & $9150(3)$ & $6484(2)$ & 12791(3) & $54(1)$ \\
\hline$C(22)$ & $5029(4)$ & $4079(2)$ & $6589(3)$ & $52(1)$ \\
\hline$C(23)$ & $5659(3)$ & $4725(1)$ & $8077(3)$ & $36(1)$ \\
\hline$C(24)$ & $6424(3)$ & $5171(1)$ & $8237(3)$ & $29(1)$ \\
\hline$C(25)$ & $6916(3)$ & $5295(1)$ & $7196(3)$ & $31(1)$ \\
\hline$C(26)$ & $7724(3)$ & $5697(2)$ & $6814(3)$ & $42(1)$ \\
\hline$C(27)$ & $8011(4)$ & $5690(2)$ & $5724(3)$ & $54(1)$ \\
\hline$C(28)$ & $7520(4)$ & $5306(2)$ & $5020(3)$ & $60(1)$ \\
\hline$C(29)$ & $6715(3)$ & 4904(2) & $5363(3)$ & $48(1)$ \\
\hline$C(30)$ & 6424(3) & $4909(2)$ & 6458(3) & $34(1)$ \\
\hline
\end{tabular}


Table 3. Bond lengths $[\AA]$ and angles $\left[{ }^{\circ}\right]$ for 03211 slm.

\begin{tabular}{|c|c|}
\hline $\mathrm{Fe}(1)-\mathrm{C}(11)$ & $1.731(4)$ \\
\hline $\mathrm{Fe}(1)-\mathrm{C}(10)$ & $1.755(4)$ \\
\hline $\mathrm{Fe}(1)-\mathrm{C}(5)$ & $2.021(3)$ \\
\hline $\mathrm{Fe}(1)-\mathrm{C}(4)$ & $2.077(3)$ \\
\hline $\mathrm{Fe}(1)-\mathrm{N}(1)$ & $2.119(3)$ \\
\hline $\mathrm{Fe}(1)-\mathrm{C}(3)$ & $2.147(3)$ \\
\hline $\mathrm{Fe}(1)-\mathrm{B}(1)$ & $2.314(4)$ \\
\hline $\mathrm{Fe}(1)-\mathrm{Si}(1)$ & $2.3264(11)$ \\
\hline$S(1)-C(17)$ & $1.768(3)$ \\
\hline$S(1)-C(18)$ & $1.846(4)$ \\
\hline $\operatorname{Si}(1)-C(12)$ & $1.865(4)$ \\
\hline $\operatorname{Si}(1)-C(13)$ & $1.879(4)$ \\
\hline $\operatorname{Si}(1)-C(14)$ & $1.890(4)$ \\
\hline $\mathrm{N}(1)-\mathrm{C}(5)$ & $1.417(4)$ \\
\hline $\mathrm{N}(1)-\mathrm{B}(1)$ & $1.462(5)$ \\
\hline $\mathrm{N}(1)-\mathrm{C}(6)$ & $1.511(4)$ \\
\hline $\mathrm{N}(2)-\mathrm{C}(23)$ & $1.366(4)$ \\
\hline $\mathrm{N}(2)-\mathrm{C}(30)$ & $1.385(4)$ \\
\hline $\mathrm{N}(2)-\mathrm{C}(22)$ & $1.458(4)$ \\
\hline $\mathrm{O}(1)-\mathrm{B}(1)$ & $1.380(4)$ \\
\hline $\mathrm{O}(1)-\mathrm{C}(15)$ & $1.451(4)$ \\
\hline $\mathrm{O}(2)-\mathrm{C}(11)$ & $1.166(4)$ \\
\hline $\mathrm{O}(3)-\mathrm{C}(10)$ & $1.153(4)$ \\
\hline $\mathrm{O}(4)-\mathrm{C}(17)$ & $1.217(4)$ \\
\hline $\mathrm{B}(1)-\mathrm{C}(3)$ & $1.530(5)$ \\
\hline$C(3)-C(4)$ & $1.407(5)$ \\
\hline$C(4)-C(5)$ & $1.382(5)$ \\
\hline$C(6)-C(7)$ & $1.519(5)$ \\
\hline$C(6)-C(8)$ & $1.519(5)$ \\
\hline$C(6)-C(9)$ & $1.518(5)$ \\
\hline$C(15)-C(24)$ & $1.479(5)$ \\
\hline$C(15)-C(16)$ & $1.531(4)$ \\
\hline$C(16)-C(17)$ & $1.504(5)$ \\
\hline $\mathrm{C}(18)-\mathrm{C}(19)$ & $1.517(5)$ \\
\hline$C(18)-C(21)$ & $1.518(5)$ \\
\hline$C(18)-C(20)$ & $1.533(5)$ \\
\hline$C(23)-C(24)$ & $1.377(5)$ \\
\hline$C(24)-C(25)$ & $1.436(4)$ \\
\hline$C(25)-C(26)$ & $1.404(5)$ \\
\hline$C(25)-C(30)$ & $1.405(5)$ \\
\hline$C(26)-C(27)$ & $1.388(5)$ \\
\hline$C(27)-C(28)$ & $1.377(6)$ \\
\hline$C(28)-C(29)$ & $1.385(5)$ \\
\hline C(29)-C(30) & $1.395(5)$ \\
\hline
\end{tabular}




\begin{tabular}{|c|c|}
\hline $\mathrm{C}(11)-\mathrm{Fe}(1)-\mathrm{C}(10)$ & $97.48(16)$ \\
\hline$C(11)-F e(1)-C(5)$ & $98.04(16)$ \\
\hline$C(10)-F e(1)-C(5)$ & $156.77(14)$ \\
\hline $\mathrm{C}(11)-\mathrm{Fe}(1)-\mathrm{C}(4)$ & $126.16(15)$ \\
\hline $\mathrm{C}(10)-\mathrm{Fe}(1)-\mathrm{C}(4)$ & $134.69(16)$ \\
\hline$C(5)-F e(1)-C(4)$ & $39.39(14)$ \\
\hline $\mathrm{C}(11)-\mathrm{Fe}(1)-\mathrm{N}(1)$ & $104.00(14)$ \\
\hline $\mathrm{C}(10)-\mathrm{Fe}(1)-\mathrm{N}(1)$ & $118.93(13)$ \\
\hline $\mathrm{C}(5)-\mathrm{Fe}(1)-\mathrm{N}(1)$ & $39.94(11)$ \\
\hline $\mathrm{C}(4)-\mathrm{Fe}(1)-\mathrm{N}(1)$ & $66.21(13)$ \\
\hline $\mathrm{C}(11)-\mathrm{Fe}(1)-\mathrm{C}(3)$ & $164.18(15)$ \\
\hline$C(10)-F e(1)-C(3)$ & $98.30(15)$ \\
\hline$C(5)-F e(1)-C(3)$ & $66.62(15)$ \\
\hline $\mathrm{C}(4)-\mathrm{Fe}(1)-\mathrm{C}(3)$ & $38.87(13)$ \\
\hline $\mathrm{N}(1)-\mathrm{Fe}(1)-\mathrm{C}(3)$ & $67.14(13)$ \\
\hline $\mathrm{C}(11)-\mathrm{Fe}(1)-\mathrm{B}(1)$ & $138.28(16)$ \\
\hline $\mathrm{C}(10)-\mathrm{Fe}(1)-\mathrm{B}(1)$ & $92.29(14)$ \\
\hline $\mathrm{C}(5)-\mathrm{Fe}(1)-\mathrm{B}(1)$ & $64.71(14)$ \\
\hline $\mathrm{C}(4)-\mathrm{Fe}(1)-\mathrm{B}(1)$ & $64.44(13)$ \\
\hline $\mathrm{N}(1)-\mathrm{Fe}(1)-\mathrm{B}(1)$ & $38.20(13)$ \\
\hline $\mathrm{C}(3)-\mathrm{Fe}(1)-\mathrm{B}(1)$ & $39.89(13)$ \\
\hline$C(11)-\mathrm{Fe}(1)-\mathrm{Si}(1)$ & $82.84(12)$ \\
\hline$C(10)-F e(1)-S i(1)$ & $85.86(11)$ \\
\hline $\mathrm{C}(5)-\mathrm{Fe}(1)-\mathrm{Si}(1)$ & $113.16(10)$ \\
\hline $\mathrm{C}(4)-\mathrm{Fe}(1)-\mathrm{Si}(1)$ & $87.99(10)$ \\
\hline $\mathrm{N}(1)-\mathrm{Fe}(1)-\mathrm{Si}(1)$ & $152.40(8)$ \\
\hline $\mathrm{C}(3)-\mathrm{Fe}(1)-\mathrm{Si}(1)$ & $99.30(10)$ \\
\hline $\mathrm{B}(1)-\mathrm{Fe}(1)-\mathrm{Si}(1)$ & $138.47(11)$ \\
\hline$C(17)-S(1)-C(18)$ & $107.36(16)$ \\
\hline$C(12)-S i(1)-C(13)$ & $106.4(2)$ \\
\hline$C(12)-S i(1)-C(14)$ & $107.7(2)$ \\
\hline$C(13)-S i(1)-C(14)$ & $105.6(2)$ \\
\hline$C(12)-S i(1)-F e(1)$ & $110.82(14)$ \\
\hline $\mathrm{C}(13)-\mathrm{Si}(1)-\mathrm{Fe}(1)$ & $113.61(14)$ \\
\hline $\mathrm{C}(14)-\mathrm{Si}(1)-\mathrm{Fe}(1)$ & $112.23(16)$ \\
\hline$C(5)-N(1)-B(1)$ & $108.3(3)$ \\
\hline$C(5)-N(1)-C(6)$ & $122.8(3)$ \\
\hline $\mathrm{B}(1)-\mathrm{N}(1)-\mathrm{C}(6)$ & $127.7(3)$ \\
\hline $\mathrm{C}(5)-\mathrm{N}(1)-\mathrm{Fe}(1)$ & $66.32(18)$ \\
\hline $\mathrm{B}(1)-\mathrm{N}(1)-\mathrm{Fe}(1)$ & 78.16(19) \\
\hline $\mathrm{C}(6)-\mathrm{N}(1)-\mathrm{Fe}(1)$ & $130.7(2)$ \\
\hline $\mathrm{C}(23)-\mathrm{N}(2)-\mathrm{C}(30)$ & 108.1(3) \\
\hline $\mathrm{C}(23)-\mathrm{N}(2)-\mathrm{C}(22)$ & $125.1(3)$ \\
\hline $\mathrm{C}(30)-\mathrm{N}(2)-\mathrm{C}(22)$ & $126.5(3)$ \\
\hline $\mathrm{B}(1)-\mathrm{O}(1)-\mathrm{C}(15)$ & 119.5(3) \\
\hline $\mathrm{O}(1)-\mathrm{B}(1)-\mathrm{N}(1)$ & 121.3(3) \\
\hline $\mathrm{O}(1)-\mathrm{B}(1)-\mathrm{C}(3)$ & $134.7(3)$ \\
\hline
\end{tabular}




$\begin{array}{ll}\mathrm{N}(1)-\mathrm{B}(1)-\mathrm{C}(3) & 104.0(3) \\ \mathrm{O}(1)-\mathrm{B}(1)-\mathrm{Fe}(1) & 135.5(2) \\ \mathrm{N}(1)-\mathrm{B}(1)-\mathrm{Fe}(1) & 63.64(18) \\ \mathrm{C}(3)-\mathrm{B}(1)-\mathrm{Fe}(1) & 64.15(18) \\ \mathrm{C}(4)-\mathrm{C}(3)-\mathrm{B}(1) & 106.2(3) \\ \mathrm{C}(4)-\mathrm{C}(3)-\mathrm{Fe}(1) & 67.9(2) \\ \mathrm{B}(1)-\mathrm{C}(3)-\mathrm{Fe}(1) & 76.0(2) \\ \mathrm{C}(5)-\mathrm{C}(4)-\mathrm{C}(3) & 110.5(3) \\ \mathrm{C}(5)-\mathrm{C}(4)-\mathrm{Fe}(1) & 68.1(2) \\ \mathrm{C}(3)-\mathrm{C}(4)-\mathrm{Fe}(1) & 73.26(19) \\ \mathrm{C}(4)-\mathrm{C}(5)-\mathrm{N}(1) & 109.9(3) \\ \mathrm{C}(4)-\mathrm{C}(5)-\mathrm{Fe}(1) & 72.5(2) \\ \mathrm{N}(1)-\mathrm{C}(5)-\mathrm{Fe}(1) & 73.74(18) \\ \mathrm{N}(1)-\mathrm{C}(6)-\mathrm{C}(7) & 106.5(3) \\ \mathrm{N}(1)-\mathrm{C}(6)-\mathrm{C}(8) & 108.0(3) \\ \mathrm{C}(7)-\mathrm{C}(6)-\mathrm{C}(8) & 111.0(3) \\ \mathrm{N}(1)-\mathrm{C}(6)-\mathrm{C}(9) & 111.7(3) \\ \mathrm{C}(7)-\mathrm{C}(6)-\mathrm{C}(9) & 109.8(3) \\ \mathrm{C}(8)-\mathrm{C}(6)-\mathrm{C}(9) & 109.9(3) \\ \mathrm{O}(3)-\mathrm{C}(10)-\mathrm{Fe}(1) & 177.2(3) \\ \mathrm{O}(2)-\mathrm{C}(11)-\mathrm{Fe}(1) & 179.0(3) \\ \mathrm{O}(1)-\mathrm{C}(15)-\mathrm{C}(24) & 112.8(3) \\ \mathrm{O}(1)-\mathrm{C}(15)-\mathrm{C}(16) & 104.3(3) \\ \mathrm{C}(24)-\mathrm{C}(15)-\mathrm{C}(16) & 113.2(3) \\ \mathrm{C}(17)-\mathrm{C}(16)-\mathrm{C}(15) & 112.3(3) \\ \mathrm{O}(4)-\mathrm{C}(17)-\mathrm{C}(16) & 122.9(3) \\ \mathrm{O}(4)-\mathrm{C}(17)-\mathrm{S}(1) & 124.8(3) \\ \mathrm{C}(16)-\mathrm{C}(17)-\mathrm{S}(1) & 112.3(2) \\ \mathrm{C}(19)-\mathrm{C}(18)-\mathrm{C}(21) & 111.8(3) \\ \mathrm{C}(19)-\mathrm{C}(18)-\mathrm{C}(20) & 109.7(3) \\ \mathrm{C}(21)-\mathrm{C}(18)-\mathrm{C}(20) & 110.4(3) \\ \mathrm{C}(19)-\mathrm{C}(18)-\mathrm{S}(1) & 110.0(3) \\ \mathrm{C}(21)-\mathrm{C}(18)-\mathrm{S}(1) & 111.2(3) \\ \mathrm{C}(20)-\mathrm{C}(18)-\mathrm{S}(1) & 103.3(2) \\ \mathrm{N}(2)-\mathrm{C}(23)-\mathrm{C}(24) & 110.8(3) \\ \mathrm{C}(23)-\mathrm{C}(24)-\mathrm{C}(25) & 105.9(3) \\ \mathrm{C}(23)-\mathrm{C}(24)-\mathrm{C}(15) & 125.4(3) \\ \mathrm{C}(25)-\mathrm{C}(24)-\mathrm{C}(15) & 128.6(3) \\ \mathrm{C}(26)-\mathrm{C}(25)-\mathrm{C}(30) & 118.7(3) \\ \mathrm{C}(26)-\mathrm{C}(25)-\mathrm{C}(24) & 134.1(3) \\ \mathrm{C}(30)-\mathrm{C}(25)-\mathrm{C}(24) & 107.1(3) \\ \mathrm{C}(27)-\mathrm{C}(26)-\mathrm{C}(25) & 118.2(4) \\ \mathrm{C}(28)-\mathrm{C}(27)-\mathrm{C}(26) & 121.8(4) \\ \mathrm{C}(29)-\mathrm{C}(28)-\mathrm{C}(27) & 121.7(4) \\ \mathrm{C}(28)-\mathrm{C}(29)-\mathrm{C}(30) & 116.6(4) \\ \mathrm{N}(2)-\mathrm{C}(30)-\mathrm{C}(29) & 129.1(3) \\ \mathrm{N}(2)-\mathrm{C}(30)-\mathrm{C}(25) & 108.0(3) \\ & \end{array}$


$C(29)-C(30)-C(25)$

122.9(3) 
Table 4. Anisotropic displacement parameters $\left(\AA^{2} \times 10^{3}\right)$ for 03211 slm. The anisotropic displacement factor exponent takes the form: $-2 p^{2}\left[h^{2} a^{* 2} U^{11}+\ldots+2 h k a^{*} b^{*} U^{12}\right]$

\begin{tabular}{|c|c|c|c|c|c|c|}
\hline & $\mathrm{U}^{11}$ & $\mathrm{U}^{22}$ & $\mathrm{U}^{33}$ & $\mathrm{U}^{23}$ & $\mathrm{U}^{13}$ & $\mathrm{U}^{12}$ \\
\hline $\mathrm{Fe}(1)$ & $25(1)$ & $34(1)$ & $27(1)$ & $3(1)$ & $7(1)$ & $4(1)$ \\
\hline$S(1)$ & $31(1)$ & $52(1)$ & $35(1)$ & $-4(1)$ & $7(1)$ & $-6(1)$ \\
\hline $\operatorname{Si}(1)$ & $27(1)$ & $50(1)$ & $52(1)$ & $7(1)$ & $0(1)$ & $0(1)$ \\
\hline $\mathrm{N}(1)$ & $28(2)$ & $35(2)$ & $29(2)$ & $-2(1)$ & $4(1)$ & $8(1)$ \\
\hline $\mathrm{N}(2)$ & $38(2)$ & $37(2)$ & $36(2)$ & $-9(2)$ & $-1(1)$ & $-7(2)$ \\
\hline $\mathrm{O}(1)$ & $27(1)$ & $29(1)$ & $35(1)$ & $-3(1)$ & $1(1)$ & $3(1)$ \\
\hline $\mathrm{O}(2)$ & $64(2)$ & $43(2)$ & $52(2)$ & $14(2)$ & $11(1)$ & $15(1)$ \\
\hline $\mathrm{O}(3)$ & $55(2)$ & $54(2)$ & $35(2)$ & $-9(1)$ & $5(1)$ & $11(1)$ \\
\hline $\mathrm{O}(4)$ & $32(1)$ & $64(2)$ & $36(2)$ & $-3(1)$ & $5(1)$ & $-6(1)$ \\
\hline $\mathrm{B}(1)$ & $32(2)$ & $38(3)$ & $19(2)$ & $3(2)$ & $3(2)$ & $3(2)$ \\
\hline$C(3)$ & $33(2)$ & $40(2)$ & $36(2)$ & $16(2)$ & $8(2)$ & $4(2)$ \\
\hline$C(4)$ & $32(2)$ & $53(3)$ & $34(2)$ & $11(2)$ & $10(2)$ & $8(2)$ \\
\hline$C(5)$ & $38(2)$ & $52(3)$ & $26(2)$ & $1(2)$ & $10(2)$ & $19(2)$ \\
\hline$C(6)$ & $43(2)$ & $33(2)$ & $41(2)$ & $-6(2)$ & $-2(2)$ & $2(2)$ \\
\hline$C(7)$ & $71(3)$ & $64(3)$ & $58(3)$ & $-14(2)$ & $-19(2)$ & $2(2)$ \\
\hline$C(8)$ & $48(2)$ & $46(3)$ & $71(3)$ & $-3(2)$ & $13(2)$ & $-12(2)$ \\
\hline C(9) & $70(3)$ & $35(2)$ & $86(3)$ & $-13(2)$ & $-4(3)$ & $6(2)$ \\
\hline$C(10)$ & $27(2)$ & $39(2)$ & $34(2)$ & $6(2)$ & $1(2)$ & $1(2)$ \\
\hline$C(11)$ & $32(2)$ & $46(3)$ & $30(2)$ & $1(2)$ & $8(2)$ & $7(2)$ \\
\hline$C(12)$ & $41(2)$ & $58(3)$ & $106(4)$ & $-3(3)$ & $-11(2)$ & $-7(2)$ \\
\hline$C(13)$ & $47(2)$ & $113(4)$ & $58(3)$ & $5(3)$ & $-16(2)$ & $1(3)$ \\
\hline$C(14)$ & $35(2)$ & $80(3)$ & $93(4)$ & $16(3)$ & $14(2)$ & $17(2)$ \\
\hline$C(15)$ & $27(2)$ & $31(2)$ & $31(2)$ & $0(2)$ & $4(2)$ & $4(2)$ \\
\hline$C(16)$ & $32(2)$ & $35(2)$ & $37(2)$ & $-7(2)$ & $1(2)$ & $7(2)$ \\
\hline$C(17)$ & $29(2)$ & $29(2)$ & $36(2)$ & $-1(2)$ & $-1(2)$ & $6(2)$ \\
\hline$C(18)$ & $28(2)$ & $45(2)$ & $37(2)$ & $-3(2)$ & $2(2)$ & $-5(2)$ \\
\hline$C(19)$ & $42(2)$ & $55(3)$ & $46(3)$ & $1(2)$ & $-1(2)$ & $1(2)$ \\
\hline$C(20)$ & $49(2)$ & $81(3)$ & $52(3)$ & $-4(3)$ & $-3(2)$ & $-28(2)$ \\
\hline$C(21)$ & $54(2)$ & $58(3)$ & $48(3)$ & $-18(2)$ & $-2(2)$ & $16(2)$ \\
\hline$C(22)$ & $50(2)$ & $48(3)$ & $58(3)$ & $-14(2)$ & $-3(2)$ & $-10(2)$ \\
\hline$C(23)$ & $38(2)$ & $37(2)$ & $32(2)$ & $1(2)$ & $3(2)$ & $2(2)$ \\
\hline$C(24)$ & $26(2)$ & $31(2)$ & $30(2)$ & $-1(2)$ & $3(2)$ & $4(2)$ \\
\hline$C(25)$ & $21(2)$ & $38(2)$ & $35(2)$ & $-1(2)$ & $3(2)$ & $2(2)$ \\
\hline$C(26)$ & $30(2)$ & $52(3)$ & $43(2)$ & $-1(2)$ & $2(2)$ & $-3(2)$ \\
\hline$C(27)$ & $40(2)$ & $77(3)$ & $47(3)$ & $3(2)$ & $14(2)$ & $-14(2)$ \\
\hline$C(28)$ & $48(2)$ & $96(4)$ & $36(3)$ & $-3(3)$ & $16(2)$ & $-9(2)$ \\
\hline$C(29)$ & $39(2)$ & $67(3)$ & $38(2)$ & $-15(2)$ & $2(2)$ & $4(2)$ \\
\hline$C(30)$ & $26(2)$ & $41(2)$ & $34(2)$ & $-4(2)$ & $3(2)$ & $2(2)$ \\
\hline
\end{tabular}


Table 5. Hydrogen coordinates $\left(x 10^{4}\right)$ and isotropic displacement parameters $\left(\AA^{2} \times 10^{3}\right)$ for 03211slm.

\begin{tabular}{|c|c|c|c|c|}
\hline & $x$ & $\mathrm{y}$ & $\mathrm{z}$ & $\mathrm{U}(\mathrm{eq})$ \\
\hline $\mathrm{H}(3)$ & 3733 & 5602 & 9130 & 44 \\
\hline $\mathrm{H}(4)$ & 2235 & 6343 & 9788 & 48 \\
\hline $\mathrm{H}(5)$ & 3439 & 7218 & 10015 & 46 \\
\hline $\mathrm{H}(7 \mathrm{~A})$ & 6157 & 7078 & 11378 & 97 \\
\hline $\mathrm{H}(7 \mathrm{~B})$ & 7410 & 7378 & 10995 & 97 \\
\hline $\mathrm{H}(7 \mathrm{C})$ & 7222 & 6749 & 10761 & 97 \\
\hline $\mathrm{H}(8 \mathrm{~A})$ & 7395 & 6835 & 8775 & 82 \\
\hline $\mathrm{H}(8 \mathrm{~B})$ & 7721 & 7460 & 8941 & 82 \\
\hline $\mathrm{H}(8 \mathrm{C})$ & 6578 & 7282 & 8120 & 82 \\
\hline $\mathrm{H}(9 \mathrm{~A})$ & 4982 & 7843 & 9116 & 96 \\
\hline $\mathrm{H}(9 \mathrm{~B})$ & 6199 & 8054 & 9813 & 96 \\
\hline $\mathrm{H}(9 \mathrm{C})$ & 5005 & 7811 & 10415 & 96 \\
\hline $\mathrm{H}(12 \mathrm{~A})$ & 581 & 5700 & 7133 & 103 \\
\hline $\mathrm{H}(12 \mathrm{~B})$ & 1296 & 5684 & 8305 & 103 \\
\hline $\mathrm{H}(12 \mathrm{C})$ & 2056 & 5538 & 7244 & 103 \\
\hline $\mathrm{H}(13 \mathrm{~A})$ & 2386 & 6443 & 5521 & 110 \\
\hline $\mathrm{H}(13 \mathrm{~B})$ & 1838 & 7028 & 5780 & 110 \\
\hline $\mathrm{H}(13 \mathrm{C})$ & 882 & 6532 & 5596 & 110 \\
\hline $\mathrm{H}(14 \mathrm{~A})$ & -251 & 6842 & 7638 & 104 \\
\hline $\mathrm{H}(14 \mathrm{~B})$ & 790 & 7292 & 7955 & 104 \\
\hline $\mathrm{H}(14 \mathrm{C})$ & 516 & 6814 & 8786 & 104 \\
\hline $\mathrm{H}(15)$ & 6142 & 5279 & 9848 & 36 \\
\hline $\mathrm{H}(16 \mathrm{~A})$ & 8312 & 4977 & 9714 & 42 \\
\hline $\mathrm{H}(16 \mathrm{~B})$ & 8663 & 5543 & 9183 & 42 \\
\hline $\mathrm{H}(19 \mathrm{~A})$ & 10331 & 5663 & 13824 & 72 \\
\hline $\mathrm{H}(19 \mathrm{~B})$ & 10841 & 5340 & 12805 & 72 \\
\hline $\mathrm{H}(19 \mathrm{C})$ & 9341 & 5391 & 12969 & 72 \\
\hline $\mathrm{H}(20 \mathrm{~A})$ & 11718 & 6432 & 13175 & 91 \\
\hline $\mathrm{H}(20 \mathrm{~B})$ & 11431 & 6679 & 11991 & 91 \\
\hline $\mathrm{H}(20 \mathrm{C})$ & 12069 & 6097 & 12120 & 91 \\
\hline $\mathrm{H}(21 \mathrm{~A})$ & 8301 & 6320 & 12733 & 80 \\
\hline $\mathrm{H}(21 \mathrm{~B})$ & 9159 & 6812 & 12347 & 80 \\
\hline $\mathrm{H}(21 \mathrm{C})$ & 9357 & 6576 & 13552 & 80 \\
\hline $\mathrm{H}(22 \mathrm{~A})$ & 5657 & 3788 & 6567 & 78 \\
\hline $\mathrm{H}(22 \mathrm{~B})$ & 4677 & 4149 & 5853 & 78 \\
\hline $\mathrm{H}(22 \mathrm{C})$ & 4341 & 3974 & 7060 & 78 \\
\hline $\mathrm{H}(23)$ & 5204 & 4552 & 8630 & 43 \\
\hline $\mathrm{H}(26)$ & 8064 & 5967 & 7289 & 50 \\
\hline $\mathrm{H}(27)$ & 8563 & 5958 & 5457 & 65 \\
\hline $\mathrm{H}(28)$ & 7739 & 5316 & 4280 & 72 \\
\hline $\mathrm{H}(29)$ & 6378 & 4638 & 4878 & 58 \\
\hline
\end{tabular}


Table 6. Torsion angles $\left[{ }^{\circ}\right]$ for 03211slm.

\begin{tabular}{|c|c|}
\hline $\mathrm{C}(11)-\mathrm{Fe}(1)-\mathrm{Si}(1)-\mathrm{C}(12)$ & $173.2(2)$ \\
\hline$C(10)-F e(1)-S i(1)-C(12)$ & $75.1(2)$ \\
\hline$C(5)-\mathrm{Fe}(1)-\mathrm{Si}(1)-\mathrm{C}(12)$ & $-91.1(2)$ \\
\hline$C(4)-F e(1)-S i(1)-C(12)$ & $-60.0(2)$ \\
\hline$N(1)-F e(1)-S i(1)-C(12)$ & $-80.2(3)$ \\
\hline $\mathrm{C}(3)-\mathrm{Fe}(1)-\mathrm{Si}(1)-\mathrm{C}(12)$ & $-22.6(2)$ \\
\hline $\mathrm{B}(1)-\mathrm{Fe}(1)-\mathrm{Si}(1)-\mathrm{C}(12)$ & $-13.7(2)$ \\
\hline$C(11)-F e(1)-S i(1)-C(13)$ & $53.4(2)$ \\
\hline $\mathrm{C}(10)-\mathrm{Fe}(1)-\mathrm{Si}(1)-\mathrm{C}(13)$ & $-44.7(2)$ \\
\hline $\mathrm{C}(5)-\mathrm{Fe}(1)-\mathrm{Si}(1)-\mathrm{C}(13)$ & $149.1(2)$ \\
\hline $\mathrm{C}(4)-\mathrm{Fe}(1)-\mathrm{Si}(1)-\mathrm{C}(13)$ & $-179.7(2)$ \\
\hline $\mathrm{N}(1)-\mathrm{Fe}(1)-\mathrm{Si}(1)-\mathrm{C}(13)$ & $160.0(2)$ \\
\hline$C(3)-F e(1)-S i(1)-C(13)$ & $-142.4(2)$ \\
\hline $\mathrm{B}(1)-\mathrm{Fe}(1)-\mathrm{Si}(1)-\mathrm{C}(13)$ & $-133.4(2)$ \\
\hline$C(11)-F e(1)-S i(1)-C(14)$ & $-66.3(2)$ \\
\hline $\mathrm{C}(10)-\mathrm{Fe}(1)-\mathrm{Si}(1)-\mathrm{C}(14)$ & $-164.4(2)$ \\
\hline $\mathrm{C}(5)-\mathrm{Fe}(1)-\mathrm{Si}(1)-\mathrm{C}(14)$ & $29.4(2)$ \\
\hline $\mathrm{C}(4)-\mathrm{Fe}(1)-\mathrm{Si}(1)-\mathrm{C}(14)$ & $60.53(19)$ \\
\hline $\mathrm{N}(1)-\mathrm{Fe}(1)-\mathrm{Si}(1)-\mathrm{C}(14)$ & $40.3(2)$ \\
\hline $\mathrm{C}(3)-\mathrm{Fe}(1)-\mathrm{Si}(1)-\mathrm{C}(14)$ & 97.86(19) \\
\hline $\mathrm{B}(1)-\mathrm{Fe}(1)-\mathrm{Si}(1)-\mathrm{C}(14)$ & $106.8(2)$ \\
\hline $\mathrm{C}(11)-\mathrm{Fe}(1)-\mathrm{N}(1)-\mathrm{C}(5)$ & $85.8(2)$ \\
\hline $\mathrm{C}(10)-\mathrm{Fe}(1)-\mathrm{N}(1)-\mathrm{C}(5)$ & $-167.3(2)$ \\
\hline $\mathrm{C}(4)-\mathrm{Fe}(1)-\mathrm{N}(1)-\mathrm{C}(5)$ & $-37.9(2)$ \\
\hline $\mathrm{C}(3)-\mathrm{Fe}(1)-\mathrm{N}(1)-\mathrm{C}(5)$ & $-80.4(2)$ \\
\hline $\mathrm{B}(1)-\mathrm{Fe}(1)-\mathrm{N}(1)-\mathrm{C}(5)$ & $-116.2(3)$ \\
\hline $\mathrm{Si}(1)-\mathrm{Fe}(1)-\mathrm{N}(1)-\mathrm{C}(5)$ & $-15.7(3)$ \\
\hline $\mathrm{C}(11)-\mathrm{Fe}(1)-\mathrm{N}(1)-\mathrm{B}(1)$ & $-158.0(2)$ \\
\hline $\mathrm{C}(10)-\mathrm{Fe}(1)-\mathrm{N}(1)-\mathrm{B}(1)$ & $-51.1(2)$ \\
\hline $\mathrm{C}(5)-\mathrm{Fe}(1)-\mathrm{N}(1)-\mathrm{B}(1)$ & 116.2(3) \\
\hline $\mathrm{C}(4)-\mathrm{Fe}(1)-\mathrm{N}(1)-\mathrm{B}(1)$ & $78.3(2)$ \\
\hline $\mathrm{C}(3)-\mathrm{Fe}(1)-\mathrm{N}(1)-\mathrm{B}(1)$ & $35.85(19)$ \\
\hline $\operatorname{Si}(1)-\mathrm{Fe}(1)-\mathrm{N}(1)-\mathrm{B}(1)$ & $100.5(2)$ \\
\hline$C(11)-F e(1)-N(1)-C(6)$ & $-27.9(3)$ \\
\hline $\mathrm{C}(10)-\mathrm{Fe}(1)-\mathrm{N}(1)-\mathrm{C}(6)$ & $79.0(3)$ \\
\hline $\mathrm{C}(5)-\mathrm{Fe}(1)-\mathrm{N}(1)-\mathrm{C}(6)$ & $-113.7(4)$ \\
\hline $\mathrm{C}(4)-\mathrm{Fe}(1)-\mathrm{N}(1)-\mathrm{C}(6)$ & $-151.6(3)$ \\
\hline $\mathrm{C}(3)-\mathrm{Fe}(1)-\mathrm{N}(1)-\mathrm{C}(6)$ & $165.9(3)$ \\
\hline $\mathrm{B}(1)-\mathrm{Fe}(1)-\mathrm{N}(1)-\mathrm{C}(6)$ & $130.1(3)$ \\
\hline $\operatorname{Si}(1)-\mathrm{Fe}(1)-\mathrm{N}(1)-\mathrm{C}(6)$ & $-129.4(2)$ \\
\hline $\mathrm{C}(15)-\mathrm{O}(1)-\mathrm{B}(1)-\mathrm{N}(1)$ & $167.1(3)$ \\
\hline $\mathrm{C}(15)-\mathrm{O}(1)-\mathrm{B}(1)-\mathrm{C}(3)$ & $-12.3(5)$ \\
\hline $\mathrm{C}(15)-\mathrm{O}(1)-\mathrm{B}(1)-\mathrm{Fe}(1)$ & $-109.9(4)$ \\
\hline $\mathrm{C}(5)-\mathrm{N}(1)-\mathrm{B}(1)-\mathrm{O}(1)$ & $-171.0(3)$ \\
\hline $\mathrm{C}(6)-\mathrm{N}(1)-\mathrm{B}(1)-\mathrm{O}(1)$ & $-3.7(5)$ \\
\hline
\end{tabular}




\begin{tabular}{|c|c|}
\hline $\mathrm{Fe}(1)-\mathrm{N}(1)-\mathrm{B}(1)-\mathrm{O}(1)$ & 129.1(3) \\
\hline $\mathrm{C}(5)-\mathrm{N}(1)-\mathrm{B}(1)-\mathrm{C}(3)$ & $8.6(4)$ \\
\hline $\mathrm{C}(6)-\mathrm{N}(1)-\mathrm{B}(1)-\mathrm{C}(3)$ & $175.8(3)$ \\
\hline $\mathrm{Fe}(1)-\mathrm{N}(1)-\mathrm{B}(1)-\mathrm{C}(3)$ & $-51.3(2)$ \\
\hline $\mathrm{C}(5)-\mathrm{N}(1)-\mathrm{B}(1)-\mathrm{Fe}(1)$ & $59.9(2)$ \\
\hline $\mathrm{C}(6)-\mathrm{N}(1)-\mathrm{B}(1)-\mathrm{Fe}(1)$ & $-132.9(3)$ \\
\hline $\mathrm{C}(11)-\mathrm{Fe}(1)-\mathrm{B}(1)-\mathrm{O}(1)$ & $-75.7(4)$ \\
\hline $\mathrm{C}(10)-\mathrm{Fe}(1)-\mathrm{B}(1)-\mathrm{O}(1)$ & $28.2(4)$ \\
\hline $\mathrm{C}(5)-\mathrm{Fe}(1)-\mathrm{B}(1)-\mathrm{O}(1)$ & $-148.4(4)$ \\
\hline $\mathrm{C}(4)-\mathrm{Fe}(1)-\mathrm{B}(1)-\mathrm{O}(1)$ & $167.8(4)$ \\
\hline $\mathrm{N}(1)-\mathrm{Fe}(1)-\mathrm{B}(1)-\mathrm{O}(1)$ & $-108.8(4)$ \\
\hline $\mathrm{C}(3)-\mathrm{Fe}(1)-\mathrm{B}(1)-\mathrm{O}(1)$ & $128.4(5)$ \\
\hline $\mathrm{Si}(1)-\mathrm{Fe}(1)-\mathrm{B}(1)-\mathrm{O}(1)$ & $114.6(3)$ \\
\hline $\mathrm{C}(11)-\mathrm{Fe}(1)-\mathrm{B}(1)-\mathrm{N}(1)$ & $33.1(3)$ \\
\hline $\mathrm{C}(10)-\mathrm{Fe}(1)-\mathrm{B}(1)-\mathrm{N}(1)$ & $137.1(2)$ \\
\hline $\mathrm{C}(5)-\mathrm{Fe}(1)-\mathrm{B}(1)-\mathrm{N}(1)$ & $-39.56(19)$ \\
\hline $\mathrm{C}(4)-\mathrm{Fe}(1)-\mathrm{B}(1)-\mathrm{N}(1)$ & $-83.4(2)$ \\
\hline $\mathrm{C}(3)-\mathrm{Fe}(1)-\mathrm{B}(1)-\mathrm{N}(1)$ & $-122.7(3)$ \\
\hline $\mathrm{Si}(1)-\mathrm{Fe}(1)-\mathrm{B}(1)-\mathrm{N}(1)$ & $-136.61(16)$ \\
\hline $\mathrm{C}(11)-\mathrm{Fe}(1)-\mathrm{B}(1)-\mathrm{C}(3)$ & $155.8(2)$ \\
\hline $\mathrm{C}(10)-\mathrm{Fe}(1)-\mathrm{B}(1)-\mathrm{C}(3)$ & $-100.2(2)$ \\
\hline $\mathrm{C}(5)-\mathrm{Fe}(1)-\mathrm{B}(1)-\mathrm{C}(3)$ & $83.2(2)$ \\
\hline $\mathrm{C}(4)-\mathrm{Fe}(1)-\mathrm{B}(1)-\mathrm{C}(3)$ & $39.3(2)$ \\
\hline $\mathrm{N}(1)-\mathrm{Fe}(1)-\mathrm{B}(1)-\mathrm{C}(3)$ & $122.7(3)$ \\
\hline $\operatorname{Si}(1)-F e(1)-B(1)-C(3)$ & $-13.9(3)$ \\
\hline $\mathrm{O}(1)-\mathrm{B}(1)-\mathrm{C}(3)-\mathrm{C}(4)$ & $169.0(4)$ \\
\hline $\mathrm{N}(1)-\mathrm{B}(1)-\mathrm{C}(3)-\mathrm{C}(4)$ & $-10.5(4)$ \\
\hline $\mathrm{Fe}(1)-\mathrm{B}(1)-\mathrm{C}(3)-\mathrm{C}(4)$ & $-61.5(2)$ \\
\hline $\mathrm{O}(1)-\mathrm{B}(1)-\mathrm{C}(3)-\mathrm{Fe}(1)$ & $-129.5(4)$ \\
\hline $\mathrm{N}(1)-\mathrm{B}(1)-\mathrm{C}(3)-\mathrm{Fe}(1)$ & $51.0(2)$ \\
\hline$C(11)-F e(1)-C(3)-C(4)$ & $21.6(6)$ \\
\hline$C(10)-F e(1)-C(3)-C(4)$ & $-162.1(2)$ \\
\hline $\mathrm{C}(5)-\mathrm{Fe}(1)-\mathrm{C}(3)-\mathrm{C}(4)$ & $36.4(2)$ \\
\hline $\mathrm{N}(1)-\mathrm{Fe}(1)-\mathrm{C}(3)-\mathrm{C}(4)$ & $80.0(2)$ \\
\hline $\mathrm{B}(1)-\mathrm{Fe}(1)-\mathrm{C}(3)-\mathrm{C}(4)$ & 114.3(3) \\
\hline $\operatorname{Si}(1)-\mathrm{Fe}(1)-\mathrm{C}(3)-\mathrm{C}(4)$ & $-74.9(2)$ \\
\hline$C(11)-F e(1)-C(3)-B(1)$ & $-92.7(5)$ \\
\hline $\mathrm{C}(10)-\mathrm{Fe}(1)-\mathrm{C}(3)-\mathrm{B}(1)$ & $83.6(2)$ \\
\hline$C(5)-F e(1)-C(3)-B(1)$ & $-78.0(2)$ \\
\hline $\mathrm{C}(4)-\mathrm{Fe}(1)-\mathrm{C}(3)-\mathrm{B}(1)$ & $-114.3(3)$ \\
\hline $\mathrm{N}(1)-\mathrm{Fe}(1)-\mathrm{C}(3)-\mathrm{B}(1)$ & $-34.38(19)$ \\
\hline $\operatorname{Si}(1)-F e(1)-C(3)-B(1)$ & $170.72(18)$ \\
\hline$B(1)-C(3)-C(4)-C(5)$ & $8.8(4)$ \\
\hline $\mathrm{Fe}(1)-\mathrm{C}(3)-\mathrm{C}(4)-\mathrm{C}(5)$ & $-58.2(3)$ \\
\hline $\mathrm{B}(1)-\mathrm{C}(3)-\mathrm{C}(4)-\mathrm{Fe}(1)$ & $67.0(2)$ \\
\hline $\mathrm{C}(11)-\mathrm{Fe}(1)-\mathrm{C}(4)-\mathrm{C}(5)$ & $-51.9(3)$ \\
\hline$C(10)-F e(1)-C(4)-C(5)$ & $146.3(2)$ \\
\hline
\end{tabular}




\begin{tabular}{|c|c|}
\hline $\mathrm{N}(1)-\mathrm{Fe}(1)-\mathrm{C}(4)-\mathrm{C}(5)$ & $38.41(19)$ \\
\hline$C(3)-F e(1)-C(4)-C(5)$ & $121.0(3)$ \\
\hline $\mathrm{B}(1)-\mathrm{Fe}(1)-\mathrm{C}(4)-\mathrm{C}(5)$ & $80.6(2)$ \\
\hline $\mathrm{Si}(1)-\mathrm{Fe}(1)-\mathrm{C}(4)-\mathrm{C}(5)$ & $-131.51(19)$ \\
\hline$C(11)-F e(1)-C(4)-C(3)$ & $-172.8(2)$ \\
\hline$C(10)-F e(1)-C(4)-C(3)$ & $25.4(3)$ \\
\hline $\mathrm{C}(5)-\mathrm{Fe}(1)-\mathrm{C}(4)-\mathrm{C}(3)$ & $-121.0(3)$ \\
\hline $\mathrm{N}(1)-\mathrm{Fe}(1)-\mathrm{C}(4)-\mathrm{C}(3)$ & $-82.5(2)$ \\
\hline $\mathrm{B}(1)-\mathrm{Fe}(1)-\mathrm{C}(4)-\mathrm{C}(3)$ & $-40.4(2)$ \\
\hline $\operatorname{Si}(1)-\mathrm{Fe}(1)-\mathrm{C}(4)-\mathrm{C}(3)$ & $107.5(2)$ \\
\hline $\mathrm{C}(3)-\mathrm{C}(4)-\mathrm{C}(5)-\mathrm{N}(1)$ & $-3.5(4)$ \\
\hline $\mathrm{Fe}(1)-\mathrm{C}(4)-\mathrm{C}(5)-\mathrm{N}(1)$ & $-64.7(2)$ \\
\hline$C(3)-C(4)-C(5)-F e(1)$ & $61.2(3)$ \\
\hline $\mathrm{B}(1)-\mathrm{N}(1)-\mathrm{C}(5)-\mathrm{C}(4)$ & $-3.7(4)$ \\
\hline $\mathrm{C}(6)-\mathrm{N}(1)-\mathrm{C}(5)-\mathrm{C}(4)$ & $-171.7(3)$ \\
\hline $\mathrm{Fe}(1)-\mathrm{N}(1)-\mathrm{C}(5)-\mathrm{C}(4)$ & $63.9(2)$ \\
\hline $\mathrm{B}(1)-\mathrm{N}(1)-\mathrm{C}(5)-\mathrm{Fe}(1)$ & $-67.6(2)$ \\
\hline $\mathrm{C}(6)-\mathrm{N}(1)-\mathrm{C}(5)-\mathrm{Fe}(1)$ & $124.4(3)$ \\
\hline$C(11)-F e(1)-C(5)-C(4)$ & $140.1(2)$ \\
\hline$C(10)-F e(1)-C(5)-C(4)$ & $-88.4(4)$ \\
\hline $\mathrm{N}(1)-\mathrm{Fe}(1)-\mathrm{C}(5)-\mathrm{C}(4)$ & $-117.7(3)$ \\
\hline $\mathrm{C}(3)-\mathrm{Fe}(1)-\mathrm{C}(5)-\mathrm{C}(4)$ & $-35.89(19)$ \\
\hline $\mathrm{B}(1)-\mathrm{Fe}(1)-\mathrm{C}(5)-\mathrm{C}(4)$ & $-79.8(2)$ \\
\hline $\operatorname{Si}(1)-F e(1)-C(5)-C(4)$ & $54.5(2)$ \\
\hline $\mathrm{C}(11)-\mathrm{Fe}(1)-\mathrm{C}(5)-\mathrm{N}(1)$ & $-102.2(2)$ \\
\hline $\mathrm{C}(10)-\mathrm{Fe}(1)-\mathrm{C}(5)-\mathrm{N}(1)$ & $29.3(5)$ \\
\hline $\mathrm{C}(4)-\mathrm{Fe}(1)-\mathrm{C}(5)-\mathrm{N}(1)$ & 117.7(3) \\
\hline $\mathrm{C}(3)-\mathrm{Fe}(1)-\mathrm{C}(5)-\mathrm{N}(1)$ & $81.8(2)$ \\
\hline $\mathrm{B}(1)-\mathrm{Fe}(1)-\mathrm{C}(5)-\mathrm{N}(1)$ & $37.85(19)$ \\
\hline $\operatorname{Si}(1)-\mathrm{Fe}(1)-\mathrm{C}(5)-\mathrm{N}(1)$ & $172.15(15)$ \\
\hline $\mathrm{C}(5)-\mathrm{N}(1)-\mathrm{C}(6)-\mathrm{C}(7)$ & $99.1(4)$ \\
\hline $\mathrm{B}(1)-\mathrm{N}(1)-\mathrm{C}(6)-\mathrm{C}(7)$ & $-66.4(4)$ \\
\hline $\mathrm{Fe}(1)-\mathrm{N}(1)-\mathrm{C}(6)-\mathrm{C}(7)$ & $-175.3(2)$ \\
\hline $\mathrm{C}(5)-\mathrm{N}(1)-\mathrm{C}(6)-\mathrm{C}(8)$ & $-141.6(3)$ \\
\hline $\mathrm{B}(1)-\mathrm{N}(1)-\mathrm{C}(6)-\mathrm{C}(8)$ & $52.8(4)$ \\
\hline $\mathrm{Fe}(1)-\mathrm{N}(1)-\mathrm{C}(6)-\mathrm{C}(8)$ & $-56.0(4)$ \\
\hline$C(5)-N(1)-C(6)-C(9)$ & $-20.7(5)$ \\
\hline $\mathrm{B}(1)-\mathrm{N}(1)-\mathrm{C}(6)-\mathrm{C}(9)$ & $173.7(3)$ \\
\hline $\mathrm{Fe}(1)-\mathrm{N}(1)-\mathrm{C}(6)-\mathrm{C}(9)$ & $64.9(4)$ \\
\hline $\mathrm{C}(11)-\mathrm{Fe}(1)-\mathrm{C}(10)-\mathrm{O}(3)$ & $-63(7)$ \\
\hline $\mathrm{C}(5)-\mathrm{Fe}(1)-\mathrm{C}(10)-\mathrm{O}(3)$ & $166(6)$ \\
\hline $\mathrm{C}(4)-\mathrm{Fe}(1)-\mathrm{C}(10)-\mathrm{O}(3)$ & 103(7) \\
\hline $\mathrm{N}(1)-\mathrm{Fe}(1)-\mathrm{C}(10)-\mathrm{O}(3)$ & $-173(100)$ \\
\hline $\mathrm{C}(3)-\mathrm{Fe}(1)-\mathrm{C}(10)-\mathrm{O}(3)$ & $118(7)$ \\
\hline $\mathrm{B}(1)-\mathrm{Fe}(1)-\mathrm{C}(10)-\mathrm{O}(3)$ & 158(7) \\
\hline $\mathrm{Si}(1)-\mathrm{Fe}(1)-\mathrm{C}(10)-\mathrm{O}(3)$ & $20(7)$ \\
\hline $\mathrm{C}(10)-\mathrm{Fe}(1)-\mathrm{C}(11)-\mathrm{O}(2)$ & $-7(20)$ \\
\hline
\end{tabular}




\begin{tabular}{|c|c|}
\hline$C(5)-F e(1)-C(11)-O(2)$ & $156(20)$ \\
\hline $\mathrm{C}(4)-\mathrm{Fe}(1)-\mathrm{C}(11)-\mathrm{O}(2)$ & $-174(100)$ \\
\hline $\mathrm{N}(1)-\mathrm{Fe}(1)-\mathrm{C}(11)-\mathrm{O}(2)$ & $116(20)$ \\
\hline $\mathrm{C}(3)-\mathrm{Fe}(1)-\mathrm{C}(11)-\mathrm{O}(2)$ & $169(100)$ \\
\hline $\mathrm{B}(1)-\mathrm{Fe}(1)-\mathrm{C}(11)-\mathrm{O}(2)$ & $95(20)$ \\
\hline $\mathrm{Si}(1)-\mathrm{Fe}(1)-\mathrm{C}(11)-\mathrm{O}(2)$ & $-92(20)$ \\
\hline $\mathrm{B}(1)-\mathrm{O}(1)-\mathrm{C}(15)-\mathrm{C}(24)$ & $81.9(3)$ \\
\hline $\mathrm{B}(1)-\mathrm{O}(1)-\mathrm{C}(15)-\mathrm{C}(16)$ & $-154.9(3)$ \\
\hline $\mathrm{O}(1)-\mathrm{C}(15)-\mathrm{C}(16)-\mathrm{C}(17)$ & $61.5(4)$ \\
\hline$C(24)-C(15)-C(16)-C(17)$ & $-175.6(3)$ \\
\hline $\mathrm{C}(15)-\mathrm{C}(16)-\mathrm{C}(17)-\mathrm{O}(4)$ & $40.5(5)$ \\
\hline$C(15)-C(16)-C(17)-S(1)$ & $-140.2(3)$ \\
\hline $\mathrm{C}(18)-\mathrm{S}(1)-\mathrm{C}(17)-\mathrm{O}(4)$ & $9.0(4)$ \\
\hline$C(18)-S(1)-C(17)-C(16)$ & $-170.3(2)$ \\
\hline$C(17)-S(1)-C(18)-C(19)$ & $60.1(3)$ \\
\hline$C(17)-S(1)-C(18)-C(21)$ & $-64.4(3)$ \\
\hline$C(17)-S(1)-C(18)-C(20)$ & $177.2(3)$ \\
\hline $\mathrm{C}(30)-\mathrm{N}(2)-\mathrm{C}(23)-\mathrm{C}(24)$ & $0.5(4)$ \\
\hline $\mathrm{C}(22)-\mathrm{N}(2)-\mathrm{C}(23)-\mathrm{C}(24)$ & $173.9(3)$ \\
\hline$N(2)-C(23)-C(24)-C(25)$ & $0.0(4)$ \\
\hline$N(2)-C(23)-C(24)-C(15)$ & $-176.8(3)$ \\
\hline $\mathrm{O}(1)-\mathrm{C}(15)-\mathrm{C}(24)-\mathrm{C}(23)$ & $-130.8(3)$ \\
\hline$C(16)-C(15)-C(24)-C(23)$ & 111.1(4) \\
\hline $\mathrm{O}(1)-\mathrm{C}(15)-\mathrm{C}(24)-\mathrm{C}(25)$ & $53.1(4)$ \\
\hline$C(16)-C(15)-C(24)-C(25)$ & $-65.0(4)$ \\
\hline$C(23)-C(24)-C(25)-C(26)$ & $179.2(4)$ \\
\hline$C(15)-C(24)-C(25)-C(26)$ & $-4.1(6)$ \\
\hline$C(23)-C(24)-C(25)-C(30)$ & $-0.6(4)$ \\
\hline$C(15)-C(24)-C(25)-C(30)$ & 176.1(3) \\
\hline$C(30)-C(25)-C(26)-C(27)$ & $-0.8(5)$ \\
\hline$C(24)-C(25)-C(26)-C(27)$ & $179.5(4)$ \\
\hline$C(25)-C(26)-C(27)-C(28)$ & $0.5(6)$ \\
\hline$C(26)-C(27)-C(28)-C(29)$ & $-0.2(6)$ \\
\hline$C(27)-C(28)-C(29)-C(30)$ & $0.2(6)$ \\
\hline $\mathrm{C}(23)-\mathrm{N}(2)-\mathrm{C}(30)-\mathrm{C}(29)$ & $179.4(4)$ \\
\hline$C(22)-N(2)-C(30)-C(29)$ & $6.2(6)$ \\
\hline$C(23)-N(2)-C(30)-C(25)$ & $-0.9(4)$ \\
\hline $\mathrm{C}(22)-\mathrm{N}(2)-\mathrm{C}(30)-\mathrm{C}(25)$ & $-174.1(3)$ \\
\hline$C(28)-C(29)-C(30)-N(2)$ & $179.2(3)$ \\
\hline$C(28)-C(29)-C(30)-C(25)$ & $-0.5(5)$ \\
\hline $\mathrm{C}(26)-\mathrm{C}(25)-\mathrm{C}(30)-\mathrm{N}(2)$ & $-178.9(3)$ \\
\hline $\mathrm{C}(24)-\mathrm{C}(25)-\mathrm{C}(30)-\mathrm{N}(2)$ & $0.9(4)$ \\
\hline$C(26)-C(25)-C(30)-C(29)$ & $0.8(5)$ \\
\hline$C(24)-C(25)-C(30)-C(29)$ & $-179.4(3)$ \\
\hline
\end{tabular}




\section{Mechanistic Studies}

Sample procedure for examining the order of the reaction in each reaction component (for the general procedure, see Section 4: entry 4 of Table 1). The Mukaiyama aldol reactions were monitored by ${ }^{1} \mathrm{H}$ NMR at $20{ }^{\circ} \mathrm{C}$. Iron complex $( \pm)-1$ (21.0 $\mathrm{mg}, 0.0440 \mathrm{mmol})$, indole-3-carboxaldehyde $(14.0 \mathrm{mg}, 0.880 \mathrm{mmol})$, and hexamethylbenzene (internal standard; $4.5 \mathrm{mg}, 0.028 \mathrm{mmol}$ ) were dissolved in $\mathrm{CD}_{3} \mathrm{CN}$ $(1.23 \mathrm{~mL})$, thereby generating a solution that was $0.0358 \mathrm{M}$ in $( \pm)-1$ and $0.0715 \mathrm{M}$ in indole-3-carboxaldehyde ("Solution A"). Solution A $(0.35 \mathrm{~mL})$ was added to each of three J. Young NMR tubes, followed by the nucleophile [4.0 $\mu \mathrm{L}(0.013 \mathrm{mmol}), 8.5 \mu \mathrm{L}$ $(0.028 \mathrm{mmol}), 17.0 \mu \mathrm{L}(0.0560 \mathrm{mmol})]$. The time of mixing was taken as $\mathrm{t}_{0}$. The conversion of reactants to products at $20^{\circ} \mathrm{C}$ was determined by integrating the resonances corresponding to the trimethylsilyl group of $( \pm)-\mathbf{1}$ versus the internal standard. The data are graphed below. 

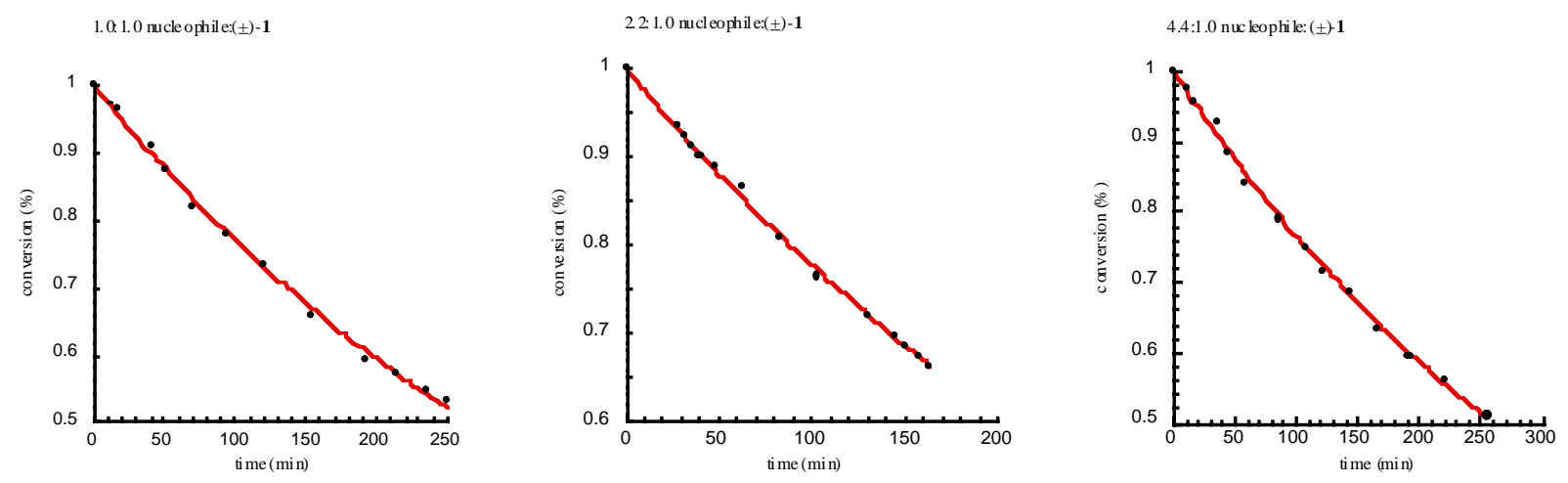

Variation in the concentration of aldehyde
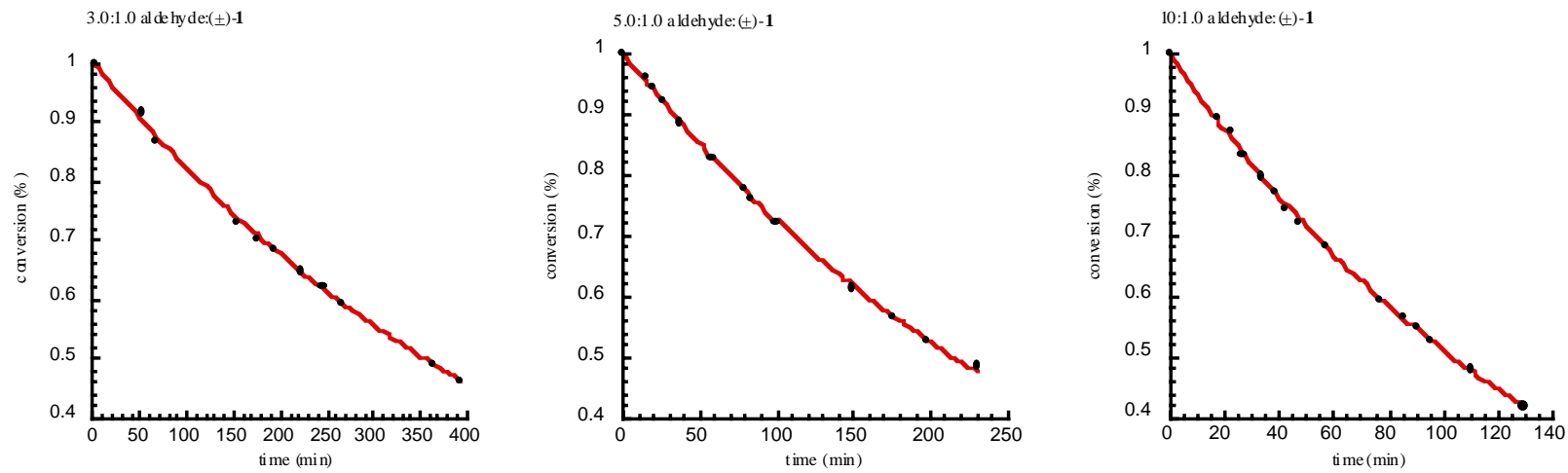

Variation in the concentration of $( \pm)-1$
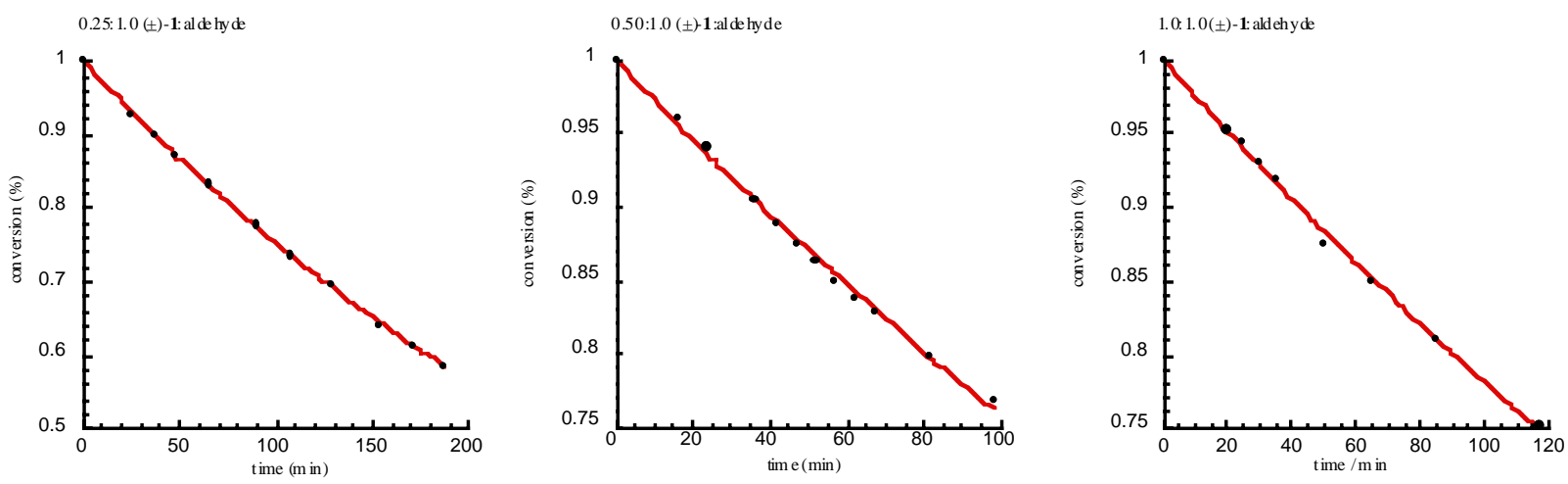


\section{References}

(1) Liu, S.-Y.; Lo, M. M.-C.; Fu, G. C. Angew. Chem., Int. Ed. 2002, 41, 174-176.

(2) Davis, A. P.; Plunkett, S. J.; Muir, J. E. Chem. Commun. 1998, 1797-1798.

(3) Ishitani, H.; Yamashita, Y.; Shimizu, H.; Kobayashi, S. J. Am. Chem. Soc. 2000, 122, 5403-5404. 